\title{
CARBON STARS IN THE HAMBURG/ESO SURVEY: ABUNDANCES ${ }^{1}$
}

\author{
Judith G. Cohen, ${ }^{2}$ Andrew McWilliam, ${ }^{3}$ Stephen Shectman, ${ }^{3}$ Ian Thompson, ${ }^{3}$ Norbert Christlieb, ${ }^{4}$ \\ Jorge Melendez, ${ }^{2}$ Solange Ramirez, ${ }^{5}$ Amber Swensson, ${ }^{2}$ and Franz-Josef Zickgraf ${ }^{4}$ \\ Received 2005 December 13; accepted 2006 March 21
}

\begin{abstract}
We have carried out a detailed abundance analysis using high-dispersion spectra from HIRES at Keck for a sample of 16 carbon stars found among candidate extremely metal-poor (EMP) stars from the Hamburg/ESO Survey (HES). We find that the Fe metallicities for the cooler $\mathrm{C}$ stars $\left(T_{\text {eff }} \sim 5100 \mathrm{~K}\right)$ have been underestimated by a factor of $\sim 10$ by the standard HES tools. The results presented here provided crucial supporting data used recently by Cohen et al. to derive the frequency of $\mathrm{C}$ stars among EMP stars. C enhancement in these EMP C stars appears to be independent of Fe metallicity and approximately constant at $\sim \frac{1}{5}$ the solar $\epsilon(\mathrm{C})$. The $\mathrm{C}$ enhancement shows some evidence of decreasing with decreasing $T_{\text {eff }}$ (increasing luminosity), presumably due to mixing and dredge-up of C-depleted material. The mostly low ${ }^{12} \mathrm{C} /{ }^{13} \mathrm{C}$ ratios $(\sim 4)$ and the high $\mathrm{N}$ abundances in many of these stars suggest that material that has been through proton burning via the $\mathrm{CN}$ cycle comprises most of the stellar envelope. $\mathrm{C}$ enhancement in this sample is associated with strong enrichment of heavy nuclei beyond the Fe peak for 12 of the 16 stars. The remaining $\mathrm{C}$ stars from the HES, which tend to be the most Fe-poor, show no evidence for enhancement of the heavy elements. Very high enhancements of lead are detected in some of the $\mathrm{C}$ stars with highly enhanced $\mathrm{Ba}$. The strong lead lines, the high $\mathrm{Ba} / \mathrm{Eu}$ ratios, and the high ratios of abundances of the diagnostic elements in the first and second $s$-process peaks demonstrate that the $s$-process is responsible for the enhancement of the heavy elements for the majority of the $\mathrm{C}$ stars in our sample. The low ${ }^{12} \mathrm{C} /{ }^{13} \mathrm{C}$ ratios and large $\mathrm{C}$ and $\mathrm{N}$ enhancements of the EMP $\mathrm{C}$ stars are more extreme than those of intrinsic asymptotic giant branch $\mathrm{C}$ stars of near-solar Fe metallicity, but closer to the composition of $\mathrm{CH}$ stars. Our subsample of EMP C stars without s-process enhancement is reminiscent of the R-type C stars in the solar neighborhood; thus, we expect that they are formed by similar mechanisms. We suggest that both the $s$-processenhanced and Ba-normal $\mathrm{C}$ stars result from phenomena associated with mass transfer in binary systems. This leads directly to the progression from $\mathrm{C}$ stars to $\mathrm{CH}$ stars and then to Ba stars as the Fe metallicity increases.
\end{abstract}

Key words: Galaxy: halo — stars: abundances — stars: carbon

Online material: machine-readable tables

\section{INTRODUCTION}

We are engaged in a large-scale project to find extremely metal-poor (EMP) stars, characterized by $[\mathrm{Fe} / \mathrm{H}] \leq-3.0 \mathrm{dex},{ }^{6}$ by exploiting the Hamburg/ESO Survey (HES) database. The HES is an objective prism survey from which it is possible to efficiently select a variety of interesting stellar objects, among them EMP stars (Christlieb 2003; see also the review of Beers \& Christlieb 2005). The discovery of a number of very metal-poor, carbon-rich objects with diverse additional peculiarities, particularly $s$-process or/and $r$-process enrichment, and the discovery of the most iron-poor star known, HE 0107-5240 (Christlieb et al. 2004), at $[\mathrm{Fe} / \mathrm{H}]=-5.3$, which is also very C-rich and was recently surpassed by HE 1327-2326, with similar character-

\footnotetext{
${ }^{1}$ Based in part on observations obtained at the W. M. Keck Observatory, which is operated jointly by the California Institute of Technology, the University of California, and the National Aeronautics and Space Administration.

2 Palomar Observatory, California Institute of Technology, MS 105-24, Pasadena, CA 91125; jlc@astro.caltech.edu, aswenson@caltech.edu.

${ }^{3}$ Carnegie Observatories of Washington, 813 Santa Barbara Street, Pasadena, CA91101; andy@ociw.edu, shec@ociw.edu, ian@ociw.edu.

${ }^{4}$ Hamburger Sternwarte, Universität Hamburg, Gojenbergsweg 112, D-21029 Hamburg, Germany; nchristlieb@hs.uni-hamburg.de, fzickgraf@hs uni-hamburg.de.

${ }^{5}$ Spitzer Science Center, California Institute of Technology, MS 100-22, Pasadena, CA 91125; solange@ipac.caltech.edu.

${ }^{6}$ The standard nomenclature is adopted; the abundance of element $\mathrm{X}$ is given by $\epsilon(\mathrm{X})=N(\mathrm{X}) / N(\mathrm{H})$ on a scale where $N(\mathrm{H})=10^{12} \mathrm{H}$ atoms. Then $[\mathrm{X} / \mathrm{H}]=$ $\log [N(\mathrm{X}) / N(\mathrm{H})]-\log [N(\mathrm{X}) / N(\mathrm{H})]_{\odot}$, and similarly for $[\mathrm{X} / \mathrm{Fe}]$.
}

istics at $[\mathrm{Fe} / \mathrm{H}] \sim-5.6$ dex (Frebel et al. 2005), as well as the known C-rich binary $\mathrm{M}$ dwarf G77-61 established by Plez \& Cohen (2005) to have $[\mathrm{Fe} / \mathrm{H}] \sim-4$ dex, all contribute to a renewed interest in EMP carbon-rich halo stars.

Broadly speaking, when $\epsilon(\mathrm{O})$ exceeds $\epsilon(\mathrm{C})$ in cool stars, the oxide molecules $(\mathrm{CO}, \mathrm{TiO}$, etc. $)$ dominate in the outer layers of the stellar atmosphere. (This is the normal condition for solar abundance ratios.) However, if $\epsilon(\mathrm{C})$ is larger than $\epsilon(\mathrm{O})$, after the formation of $\mathrm{CO}$, extra $\mathrm{C}$ remains rather than extra $\mathrm{O}$, and carbon compounds such as $\mathrm{C}_{2}, \mathrm{CH}$, and $\mathrm{CN}$ dominate. The strong bands of $\mathrm{C}_{2}$ are then prominent in the optical spectrum of such stars, if they are cool enough, hence the origin of the name carbon stars (C stars). Our operational definition of a $\mathrm{C}$ star is one whose spectrum shows the blue-degraded band of $\mathrm{C}_{2}$ at $5160 \AA$, which is the most prominent band of this molecule within the wavelength range of the spectra discussed here. If no $\mathrm{C}_{2}$ bands are detected, but $[\mathrm{C} / \mathrm{Fe}]>1$ dex, we denote a star to be $\mathrm{C}$-enhanced. The strength of the $\mathrm{C}_{2}$ bands is a function of $T_{\text {eff }}, \epsilon(\mathrm{C})$, and to a lesser extent, $\log g,[\mathrm{Fe} / \mathrm{H}]$, and $\epsilon(\mathrm{O})$.

The purpose of the present paper is to carry out detailed chemical abundance analyses of a sample of 16 EMP C stars selected from the HES. This provides a broad database to establish the $\mathrm{Fe}$ metallicity for EMP C stars. The results presented here provided crucial supporting data used by Cohen et al. (2005b) to derive the frequency of $\mathrm{C}$ stars among EMP stars. We use the abundance ratios derived here for EMP $\mathrm{C}$ stars to discuss the origin of the $\mathrm{C}$-star phenomenon among EMP stars, which we attribute in toto to phenomena associated with binary systems. 
TABLE 1

The Sample of C Stars Selected as EMP Candidates from the HeS, Mostly from the Palomar Sample

\begin{tabular}{|c|c|c|c|c|c|c|c|}
\hline \multirow[b]{2}{*}{ ID } & \multicolumn{2}{|c|}{ Coordinates } & \multirow{2}{*}{$\begin{array}{c}V^{\mathrm{a}} \\
(\mathrm{mag})\end{array}$} & \multirow{2}{*}{$\begin{array}{c}I^{\mathrm{a}} \\
(\mathrm{mag})\end{array}$} & \multirow[b]{2}{*}{$\mathrm{JD}(2,450,000+)$} & \multirow{2}{*}{$\begin{array}{c}v_{r}^{\mathrm{b}} \\
\left(\mathrm{km} \mathrm{s}^{-1}\right)\end{array}$} & \multirow{2}{*}{$\begin{array}{c}{[\mathrm{Fe} / \mathrm{H}](\mathrm{HES})} \\
(\mathrm{dex})\end{array}$} \\
\hline & R.A. (J2000.0) & Decl. (J2000.0) & & & & & \\
\hline HE $0007-1832 \ldots \ldots \ldots \ldots \ldots \ldots$ & 000952.8 & -181612 & 15.462 & 14.831 & $\mathrm{c}$ & & \\
\hline 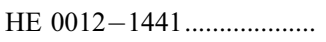 & 001527.1 & -142437 & 16.358 & 15.704 & 2547.8627 & $+11^{\mathrm{d}}$ & -2.61 \\
\hline HE $0058-0244^{\mathrm{e}} \ldots \ldots \ldots \ldots \ldots . . . . .$. & 010053.0 & -022820 & 13.727 & 12.933 & 2179.0096 & -68.4 & -2.81 \\
\hline 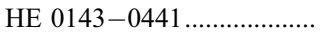 & 014537.8 & -042643 & 16.382 & 15.724 & 2547.0190 & +121.8 & -2.94 \\
\hline HE $0212-0557 \ldots \ldots \ldots \ldots \ldots \ldots$ & 021502.5 & -054323 & 14.70 & $\ldots$ & 2544.9791 & -230.6 & -3.45 \\
\hline 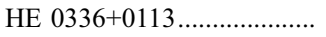 & 033852.8 & +012308 & 14.955 & 14.110 & 2179.0586 & +66.6 & -2.51 \\
\hline 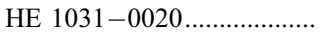 & 103424.1 & -003609 & 14.296 & 13.338 & 3152.7297 & +69.7 & -3.70 \\
\hline 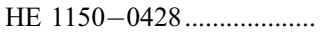 & 115306.6 & -044503 & 14.909 & 14.007 & 3152.7602 & +46.6 & -3.22 \\
\hline 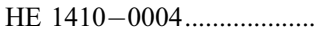 & 141304.6 & -001833 & 15.494 & 14.712 & 3534.7447 & +214.6 & -2.65 \\
\hline HE $1410+0213 \ldots \ldots \ldots \ldots \ldots \ldots$ & 141306.5 & +015921 & 13.25 & $\ldots$ & 2396.9707 & $+80^{\mathrm{f}}$ & -3.17 \\
\hline 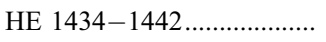 & 143726.6 & -145459 & 15.34 & $\cdots$ & 3488.9757 & $+146.9^{\mathrm{f}}$ & -3.42 \\
\hline 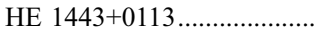 & 144616.4 & +010110 & 15.78 & $\ldots$ & 3589.7671 & -1.1 & -3.13 \\
\hline HE $1509-0806 \ldots \ldots \ldots \ldots \ldots \ldots . . . . . . .$. & 151141.5 & -081741 & 14.796 & 13.807 & 2421.9292 & -169.9 & -3.92 \\
\hline HE $2158-0348 \ldots \ldots \ldots \ldots \ldots \ldots$ & 220040.0 & -033412 & 15.707 & 14.735 & 2178.7352 & +67.6 & -2.77 \\
\hline 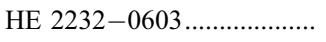 & 223447.4 & -054817 & 16.513 & 15.738 & 2178.8255 & -61.2 & -1.99 \\
\hline HE $2353-1758^{g} \ldots \ldots \ldots \ldots \ldots \ldots$ & 235612.9 & -174203 & 15.491 & 14.558 & 3641.8915 & +38.5 & -2.60 \\
\hline HE $2356-0410^{\mathrm{h}} \ldots \ldots \ldots \ldots \ldots \ldots$ & 235913.1 & -035349 & 13.622 & 12.710 & 2179.8396 & -61.5 & -3.22 \\
\hline
\end{tabular}

NoTE.-Objects observed with HIRES.

a Our photometry from ANDICAM images.

${ }^{\mathrm{b}}$ Heliocentric $v_{r}$.

${ }^{c}$ See Cohen et al. (2004).

d Double-lined spectroscopic binary, $v_{r}$ variable.

e Rediscovery of CS 22183-015.

${ }^{f}$ Only from the $\mathrm{Mg}$ triplet lines.

${ }^{g}$ HIRES spectrum obtained too late for analysis here. C star with very strong Ba II lines.

${ }^{\text {h }}$ Rediscovery of CS 22957-027.

After a description of the stellar sample in $\S 2$, readers who are not interested in the details of the abundance analyses should proceed to $\S 4$, then $\S 4.1$, and then skip to $\S 5$.

We favor scenarios of C-star formation among the EMP halo stars resulting from the evolution of binary systems, including mass transfer. The evidence supporting this is described in $\S 5$. Section 5.5 compares the derived abundances of the EMP C stars to those of various types of near-solar $[\mathrm{Fe} / \mathrm{H}]$ disk $\mathrm{C}$ stars. The implications of our hypothesis for C-star formation among the EMP stars as applied to stars of higher and lower Fe metallicity are described in $\S 6$. A brief summary concludes the paper.

\section{THE STELLAR SAMPLE}

The normal procedures outlined by Christlieb (2003) to isolate EMP stars from the candidate lists produced by the HES were followed. In brief, candidate EMP stars were selected from the HES. This was followed by vetting via moderate-resolution spectroscopy with $5 \mathrm{~m}$ class telescopes to eliminate the numerous higher abundance interlopers. The follow-up spectra for the stars discussed here were obtained either with the Double Spectrograph (Oke \& Gunn 1982) at the Hale Telescope at Mount Palomar or with the Boller \& Chivens spectrograph on the Baade and Clay Telescopes at the Las Campanas Observatory during the period from 2001 to the present.

These follow-up spectra were used to determine an estimate of the metallicity of the star, which was much more accurate than could be derived from the low-resolution objective prism spectra of the HES itself. This was accomplished via a combination of strength of absorption in $\mathrm{H} \delta$ (determining $T_{\text {eff }}$ ) and in the $\mathrm{Ca}$ II $\mathrm{K}$ line at $3933 \AA$ (the $\mathrm{KP}$ index), which determines $[\mathrm{Fe} / \mathrm{H}]$ once $T_{\text {eff }}$ and hence $\log g$ are specified. The calibration of $\mathrm{H} \delta$ plus the $\mathrm{KP}$ index to $[\mathrm{Fe} / \mathrm{H}]$ is ultimately based on the results from highresolution abundance studies of standard stars, but such calibra- tions implicitly assume that the relation between these line indices and $[\mathrm{Fe} / \mathrm{H}]$ is the same for both program and standard stars. We denote the resulting metallicity value as $[\mathrm{Fe} / \mathrm{H}](\mathrm{HES})$. The specific algorithm adopted by the HES is described in Beers et al. (1999) and is identical to that used until recently by the HK survey of Beers et al. $(1985,1992)$. Stars were chosen for observation at high resolution with HIRES (Vogt et al. 1994) at the Keck I telescope primarily on the basis of low predicted metallicity; all stars with $[\mathrm{Fe} / \mathrm{H}](\mathrm{HES}) \leq-2.9$ dex were put on the HIRES observing list, as well as selected other stars of interest.

This paper is dedicated to an exploration of those stars that turned out from their moderate-resolution spectra to be $\mathrm{C}$ stars. A more complete discussion of the selection of our C-star sample from the HES and the frequency of $\mathrm{C}$ stars within this sample will be given in J. G. Cohen et al. (2006b, in preparation). We present here detailed abundance analyses for $15 \mathrm{C}$ stars from the HES observed at the Keck I telescope. One of these is a newly discovered, short-period, double-lined spectroscopic binary. We denote this group plus the dwarf C star HE 0007-1832 discussed in Cohen et al. (2004) as the primary sample, listed in Table 1. The augmented sample also includes two C-enhanced dwarfs selected from the HES and analyzed in the same way by our group in our previously published papers: HE 0024-2523, discussed in Cohen et al. (2002), Carretta et al. (2002), and in great detail in Lucatello et al. (2003), and HE 2148-1247, discussed in Cohen et al. (2003), both of which show highly enhanced lead in their spectra, plus a third C-enhanced star whose analysis will be presented in J. G. Cohen et al. (2006a, in preparation).

Throughout this paper we ignore the two known ultra-metalpoor stars HE 0107-5240 (Christlieb et al. 2004) and HE 13272326 (Frebel et al. 2005). More than 7000 EMP candidates were searched to turn up these two stars, and there are no stars in the Galaxy known to us with $-5.2 \mathrm{dex} \leq[\mathrm{Fe} / \mathrm{H}] \leq-4.3 \mathrm{dex}$. Although 
TABLE 2

Stellar Parameters and Observations

\begin{tabular}{|c|c|c|c|c|c|c|}
\hline ID & $\begin{array}{l}T_{\text {eff }} \\
(\mathrm{K})\end{array}$ & $\begin{array}{l}\log g \\
(\operatorname{dex})\end{array}$ & $\begin{array}{c}v_{t} \\
\left(\mathrm{~km} \mathrm{~s}^{-1}\right)\end{array}$ & $\begin{array}{l}\text { Exp. Time (HIRES) } \\
\text { (s) }\end{array}$ & $\mathrm{S} / \mathrm{N}^{\mathrm{a}}$ & Source/Follow-Up \\
\hline HE $0012-1441 \ldots \ldots$ & $5730^{\mathrm{b}}$ & 3.5 & 1.6 & 10800 & 75 & Magellan \\
\hline HE $0058-0244 \ldots \ldots \ldots \ldots$ & 5620 & 3.4 & 1.6 & 4800 & 100 & Magellan \\
\hline HE $0143-0441 \ldots \ldots$ & 6240 & 3.7 & 1.6 & 9600 & 80 & P200 \\
\hline 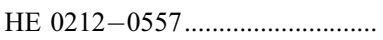 & 5075 & 2.15 & 1.8 & 6000 & 100 & P200 \\
\hline HE 0336+0113 & 5700 & 3.5 & 1.6 & 12200 & 100 & Magellan \\
\hline HE 1031-0020 & 5080 & 2.2 & 1.6 & 2400 & 80 & P200 \\
\hline HE $1150-0428 \ldots \ldots \ldots$ & 5200 & 2.55 & 1.6 & 2400 & 70 & P200 \\
\hline HE 1410-0004 ............................ & 5605 & 3.5 & 1.6 & $2400^{\mathrm{c}}$ & 60 & P200 \\
\hline HE $1410+0213 \ldots \ldots \ldots \ldots$ & 4985 & 2.0 & 1.8 & 2700 & 80 & P200 \\
\hline 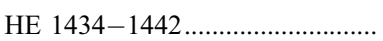 & 5420 & 3.15 & 1.6 & $6000^{\mathrm{d}}$ & 73 & P200 \\
\hline HE $1443+0113$ & 4945 & 1.95 & 1.8 & $550^{\mathrm{d}, \mathrm{e}}$ & Low & P200 \\
\hline HE $1509-0806 \ldots \ldots \ldots$ & 5185 & 2.5 & 1.6 & 2400 & 70 & P200 \\
\hline HE 2158-0348 & 5215 & 2.5 & 1.6 & 14400 & 100 & Magellan \\
\hline HE 2232-0603 ............................ & 5750 & 3.5 & 1.6 & 18000 & 90 & Magellan \\
\hline HE 2356-0410 & 5205 & 2.5 & 1.8 & 6000 & 100 & Magellan \\
\hline
\end{tabular}

${ }^{\text {a }} \mathrm{S} / \mathrm{N}$ in the continuum near $4570 \AA \AA$ per 4 pixel spectral resolution element.

${ }^{b}$ Assumes the second component does not affect the observed colors.

${ }^{c}$ HIRES-R spectrum with new mosaic CCD detector.

${ }^{\mathrm{d}}$ HIRES-B spectrum with new mosaic CCD detector.

${ }^{\mathrm{e}}$ Only one short exposure available, stopped by fog.

both of the known ultra-metal-poor stars are $\mathrm{C}$ stars with extremely large $\mathrm{C}$ enhancements, we are not certain that they represent a continuation toward lower Fe metallicities of the stars discussed here, and hence we have chosen to not consider them here.

\subsection{Stellar Parameters}

In order to determine stellar parameters for these stars, particularly the cooler ones, ideally we would compute a special set of model atmospheres that would have the abundances, particularly those of CNO, set to values appropriate for each star. This would ensure that a proper accounting of the molecular absorption would be made. We have not done this. Instead we have followed our normal procedures described in Cohen et al. (2002) of matching observed broadband photometry $V-I, V-J$, and $V-K$ to predicted grids of synthetic colors by Houdashelt et al. (2000). Cohen et al. (2002) demonstrate that there is good agreement between the Kurucz and MARCS temperature scale. They find that the $V-K$ relations of Alonso et al. $(1996,1999)$ extrapolated to EMP stars give $T_{\text {eff }}$ values $\sim 100 \mathrm{~K}$ cooler than those adopted here for giants, while the deduced $T_{\text {eff }}$ from $V-K$ for stars near the main-sequence turnoff are in good agreement.

We then rely on an appropriate $12 \mathrm{Gyr}$ isochrone from the grid of Yi et al. (2001) to obtain the surface gravity for each star. The resulting stellar parameters, which have been derived with no reference to the spectra themselves, are given in Table 2. By using the larger wavelength differences of $V-I, V-J$, and $V-K$ to determine our $T_{\text {eff }}$ values, avoiding $B-V$ and $J-K$, we achieve consistency to within $\pm 150 \mathrm{~K}$ between the $T_{\text {eff }}$ determinations from each of these three colors for all stars. We have noticed that the $B-V$ colors of the HES C stars appear too red. This behavior is expected, since the flux in the $B$ band is reduced much more by molecular bands in $C$ stars than is the flux in the $V$ band. $B-V$ colors thus tend to give a $T_{\text {eff }}$ that is too low, presumably due to the effect of molecular absorption in one or both of the filter bandpasses altering a color that because of its small wavelength coverage is, even under the best of circumstances, relatively insensitive to $T_{\text {eff }}$. This problem with $B-V$ colors was pointed out by, among others, Preston \& Sneden (2001). $J-K$ is not very sensitive to $T_{\text {eff }}$, changing in color by only $0.02 \mathrm{mag}$ for $\Delta T_{\text {eff }}$ of $100 \mathrm{~K}$. Given that many of the HES stars are sufficiently faint that the errors in their Two Micron All Sky Survey (2MASS) photometry exceed $0.05 \mathrm{mag}$ at $K$, we avoid the use of $J-K$ colors here.

The IR photometry we use is taken from 2MASS (Skrutskie et al. 2006; Cutri et al. 2003). We have obtained new photometry at $V$ and $I$ for many of the stars in our sample. We use ANDICAM images taken for this purpose over the past year via a service observing queue on the $1.3 \mathrm{~m}$ telescope at Cerro Tololo InterAmerican Observatory operated by the SMARTS consortium. ANDICAM is a dual-channel camera constructed by the Ohio State University instrument group. ${ }^{7}$ Our ANDICAM program requires photometric conditions, and additional standard star fields, charged to our ANDICAM allocation through NOAO, are always taken for us.

Our new ANDICAM photometry for our sample of C stars from the HES, as well as other relevant observational data for these stars, is presented in Table 1. Table 2 gives the resulting stellar parameters for these stars.

The uncertainty in $\log g$ arising from our $150 \mathrm{~K}$ uncertainty in $T_{\text {eff }}$ depends on the slope of the relationship between $T_{\text {eff }}$ and $\log g$ along the adopted isochrone. For stars close to the mainsequence turnoff and for subgiants, this is small, and the uncertainty in $\log g$ is 0.1 dex. However, for stars along the red giant branch (RGB), it reaches 0.4 dex.

\section{HIRES OBSERVATIONS AND ABUNDANCE ANALYSIS}

Observations with HIRES at the Keck I telescope were obtained during several runs from 2001 September to 2005 June. The weather conditions varied from night to night. A spectral resolution of 45,000 was achieved using a 0".86 wide slit projecting to 3 pixels in the HIRES focal plane CCD detector. For

\footnotetext{
7 See http://www.astronomy.ohio-state.edu/ANDICAM and http://www.astro .yale.edu/smarts.
} 
TABLE 3

Equivalent Widths for the First Five Stars of the Primary Sample of C Stars from the HeS

\begin{tabular}{|c|c|c|c|c|c|c|c|c|}
\hline \multirow{2}{*}{$\begin{array}{c}\text { LINE } \lambda \\
(\AA)\end{array}$} & \multirow[b]{2}{*}{ SPECIES } & \multirow{2}{*}{$\begin{array}{c}\mathrm{EP} \\
(\mathrm{eV})\end{array}$} & \multirow{2}{*}{$\begin{array}{c}\log (g f) \\
(\operatorname{dex})\end{array}$} & \multicolumn{5}{|c|}{$W_{\lambda}(\mathrm{m} \AA)$} \\
\hline & & & & HE $0012-1441^{\mathrm{a}}$ & HE $0058-0244$ & HE $0143-0441$ & HE $0212-0557$ & HE $0336+0113$ \\
\hline $4057.52 \ldots \ldots \ldots \ldots \ldots \ldots$ & Mg I & 4.34 & -1.200 & 34.0 & 19.2 & 21.2 & $\ldots$ & 34.4 \\
\hline $4703.00 \ldots \ldots \ldots \ldots \ldots \ldots$ & $\mathrm{Mg}_{\mathrm{I}}$ & 4.34 & -0.670 & 100.7 & $\ldots$ & $\ldots$ & $\ldots$ & $\ldots$ \\
\hline $5172.70 \ldots \ldots \ldots \ldots \ldots \ldots$ & Mg I & 2.71 & -0.380 & 258.9 & 144.7 & 163.5 & 183.5 & 210.9 \\
\hline $5183.62 \ldots$ & $\mathrm{Mg}_{\mathrm{I}}$ & 2.72 & -0.160 & 357.5 & 172.1 & 187.8 & $\ldots$ & 340.9 \\
\hline $3961.52 \ldots \ldots \ldots \ldots \ldots \ldots$ & Al I & 0.00 & -0.340 & $\ldots$ & 113.2 & 77.8 & 150.0 & 117.1 \\
\hline $3905.53 \ldots \ldots \ldots \ldots \ldots \ldots$ & Si I & 1.91 & -1.040 & $\ldots$ & $\ldots$ & 239.1 & $\ldots$ & $\ldots$ \\
\hline $4226.74 \ldots \ldots \ldots \ldots \ldots$ & $\mathrm{Ca} \mathrm{I}$ & 0.00 & 0.240 & 303.0 & $\ldots$ & $\ldots$ & $\ldots$ & $\ldots$ \\
\hline $4425.44 \ldots \ldots \ldots \ldots \ldots \ldots$ & $\mathrm{Ca} \mathrm{I}$ & 1.88 & -0.360 & $\ldots$ & $\ldots$ & 25.6 & $\ldots$ & $\ldots$ \\
\hline $4435.69 \ldots \ldots \ldots \ldots \ldots$ & $\mathrm{Ca} \mathrm{I}$ & 1.89 & -0.520 & $\ldots$ & 53.4 & $\ldots$ & $\ldots$ & 20.8 \\
\hline $4454.79 \ldots \ldots \ldots \ldots \ldots \ldots$ & $\mathrm{Ca} \mathrm{I}$ & 1.90 & 0.260 & 78.1 & 81.4 & 70.0 & $\ldots$ & $\ldots$ \\
\hline
\end{tabular}

Notes.-Table 3 is published in its entirety in the electronic edition of the Astronomical Journal. A portion is shown here for guidance regarding its form and content.

${ }^{a}$ Spectrum synthesis is used throughout for this binary star; the $W_{\lambda}$ are given here as a guide.

those stars presented here with $V>15$ mag, a spectral resolution of 34,000 was used, with the exception of HE 1410-0004, which was observed at the higher spectral resolution. The spectra cover the region from 3840 to $5330 \AA$ with no gaps between orders for $\lambda<5000 \AA$, and only small gaps thereafter. Each exposure was broken up into $1200 \mathrm{~s}$ segments to expedite removal of cosmic rays. The goal was to achieve a signal-to-noise ratio $(\mathrm{S} / \mathrm{N})$ of 100 per spectral resolution element in the continuum at $4500 \AA$; a few spectra have lower $\mathrm{S} / \mathrm{N}$. This $\mathrm{S} / \mathrm{N}$ calculation uses only Poisson statistics, ignoring issues of cosmic-ray removal, night-sky subtraction, flattening, etc. The observations were carried out with the slit length aligned to the parallactic angle.

The recently installed upgraded HIRES detector designed and built by the Lick Observatory engineering staff, led by S. Vogt, was used for three C stars observed in 2005: HE 1410-0004, HE 1443+0113, and HE 1434-1442. HIRES-R was used for the first star, and HIRES-B for the other two. We thus obtain, among other desirable things, more complete spectral coverage, reaching in a single exposure from 4020 to $7800 \AA$ with HIRES-R and from 3200 to $5900 \AA$ with HIRES-B for the instrument configurations we use. Note that only for one star in the present sample does the included spectral range reach beyond $6000 \AA$. Details of the HIRES exposures, including the exposure times and the $\mathrm{S} / \mathrm{N}$ per spectral resolution element in the continuum, are given in Table 1.

This set of HIRES data was reduced using a combination of Figaro scripts and the software package MAKEE. ${ }^{8}$ Insofar as possible, both the spectral reduction and abundance analyses presented here are identical to the procedures described in our earlier paper on EMP dwarfs from the HES (Cohen et al. 2004).

\subsection{Equivalent Widths and Abundance Analysis}

The search for absorption features present in our HIRES data and the measurement of their equivalent width $\left(W_{\lambda}\right)$ was done automatically with a FORTRAN code, EWDET, developed for a globular cluster project. Details of this code and its features are given in Ramírez et al. (2001). The strong molecular bands made it impossible to use the full spectral range; selected regions were

\footnotetext{
${ }^{8}$ MAKEE was developed by T. A. Barlow specifically for reduction of Keck HIRES data. It is freely available on the World Wide Web at the Keck Observatory home page, http://www.keckobservatory.org.
}

eliminated prior to searching for absorption features. This also applied to the radial velocity determination procedure we use, described in Cohen et al. (2004). Extensive hand checking of the $W_{\lambda}$ for blending by molecular features was necessary in many cases, such as when the line profiles were frequently distorted by blends due to strong molecular blanketing or low-S/N conditions. The spectrum of HE $1410+0213$ is so severely affected by its very strong molecular bands that only the region beyond $5160 \AA$ (plus a few strong lines near $4920 \AA$ ) could be used. For HE $1443+0113$ there is only one exposure available, which had to be terminated at $550 \mathrm{~s}$ due to deteriorating weather conditions. It has a very low $\mathrm{S} / \mathrm{N}$, and only the strongest features can be measured, i.e., $\mathrm{CH}$, the $\mathrm{Na}$ doublet, the $\mathrm{Mg}$ triplet, a few Fe I lines, and two $\mathrm{Ba}$ II lines. The $W_{\lambda}$ values for this spectrum are more uncertain than those of the others presented here.

The atomic data and list of unblended lines used (ignoring those in the regions cut out due to the strong molecular bands) are identical to those of Cohen et al. (2004). We adopt $\log \epsilon(\mathrm{Fe})=$ 7.45 dex for iron following the revisions in the solar photospheric abundances suggested by Asplund et al. (2000), Prochaska et al. (2000), and Holweger (2001). Abundances were determined from equivalent widths, except for $\mathrm{C}, \mathrm{N}$ (for which we synthesized the region of the $\mathrm{CN}$ band head near $3885 \AA$ ), and $\mathrm{Pb}$. For $\mathrm{C}$ we synthesized the region of the $\mathrm{CH}$ band near $4320 \AA$, which is considerably weaker than the main band head of the $\mathrm{G}$ band near $4305 \AA$, and hence still usable even in these $C$ stars. For the coolest $C$ stars with the strongest bands, even this region off the main band head is close to saturation. The solar abundances we adopt are those of Anders \& Grevesse (1989), slightly updated as described in Cohen et al. (2004). A synthesis using our line list of CH and CN features combined with the Kurucz (1993) solar model matches the solar Fourier Transform Spectrometer spectrum of Wallace et al. (1998) with our initially adopted $\mathrm{C}$ and $\mathrm{N}$ solar abundances, $\log \epsilon(\mathrm{C})=8.59 \mathrm{dex}$ and $\log \epsilon(\mathrm{N})=7.93 \mathrm{dex}$. These are close to those of Grevesse \& Sauval (1998) but larger than those of Asplund et al. (2004, 2005), which are 0.2 dex smaller for $\mathrm{C}$ and 0.13 dex smaller for $\mathrm{N}$. Once the $\mathrm{C}$ and $\mathrm{N}$ abundances were determined for a star, we synthesized the region of the $4057 \AA \mathrm{Pb}_{\mathrm{I}}$ line to derive the $\mathrm{Pb}$ abundance.

The equivalent widths and atomic parameters used in the analysis of the primary sample of $16 \mathrm{C}$ stars selected as EMP candidates from the HES are tabulated in Tables $3-5 ; W_{\lambda}$ values for the additional redder lines seen only in the three $\mathrm{C}$ stars observed with the upgraded HIRES detector are given in Table 6. Occasionally, 
TABLE 4

Equivalent Widths for the Next Five Stars of the Primary Sample of C Stars from the HES

\begin{tabular}{|c|c|c|c|c|c|c|c|c|}
\hline \multirow{2}{*}{$\begin{array}{c}\text { LINE } \lambda \\
(\AA)\end{array}$} & \multirow[b]{2}{*}{ SPECIES } & \multirow{2}{*}{$\begin{array}{c}\text { EP } \\
(\mathrm{eV})\end{array}$} & \multirow{2}{*}{$\begin{array}{c}\log (g f) \\
(\mathrm{dex})\end{array}$} & \multicolumn{5}{|c|}{$W_{\lambda}(\mathrm{m} \AA)$} \\
\hline & & & & HE $1031-0020$ & HE $1150-0428$ & HE $1410+0213$ & HE $1410-0004$ & HE $1434-1442$ \\
\hline $4057.52 \ldots \ldots \ldots \ldots \ldots \ldots \ldots$ & Mg I & 4.34 & -1.200 & 18.0 & $\ldots$ & $\ldots$ & 13.9 & $\ldots$ \\
\hline $4703.00 \ldots \ldots \ldots \ldots \ldots \ldots$ & $\mathrm{Mg}$ I & 4.34 & -0.670 & $\ldots$ & $\ldots$ & $\ldots$ & 31.4 & $\ldots$ \\
\hline $5172.70 \ldots \ldots \ldots \ldots \ldots \ldots$ & $\mathrm{Mg}_{\mathrm{I}}$ & 2.71 & -0.380 & 160.3 & 115.2 & 218.6 & 128.6 & 191.7 \\
\hline $5183.62 \ldots$ & Mg I & 2.72 & -0.160 & 189.2 & 130.4 & 246.3 & 142.9 & 241.6 \\
\hline 3961.52 „................. & Al I & 0.00 & -0.340 & 174.5 & $\ldots$ & $\ldots$ & $\ldots$ & $\ldots$ \\
\hline $4435.69 \ldots \ldots \ldots \ldots \ldots \ldots$ & $\mathrm{Ca} \mathrm{I}$ & 1.89 & -0.520 & 55.0 & 32.4 & $\ldots$ & $\ldots$ & $\ldots$ \\
\hline $4454.79 \ldots \ldots \ldots \ldots \ldots \ldots$ & $\mathrm{Ca} \mathrm{I}$ & 1.90 & 0.260 & 110.0 & 74.0 & $\ldots$ & $\ldots$ & $\ldots$ \\
\hline $4578.56 \ldots \ldots \ldots \ldots \ldots \ldots$ & $\mathrm{Ca} \mathrm{I}$ & 2.52 & -0.558 & 36.0 & 9.0 & $\ldots$ & $\ldots$ & $\ldots$ \\
\hline $3924.53 \ldots \ldots \ldots \ldots \ldots \ldots$ & Ti I & 0.02 & -0.940 & 32.4 & $\ldots$ & $\cdots$ & $\ldots$ & $\cdots$ \\
\hline 3958.22 ................... & Ti I & 0.05 & -0.160 & 47.4 & 23.4 & $\ldots$ & $\ldots$ & $\ldots$ \\
\hline
\end{tabular}

Notes.-Table 4 is published in its entirety in the electronic edition of the Astronomical Journal. A portion is shown here for guidance regarding its form and content.

for crucial elements for which no line was securely detected in a star, we tabulate upper limits to $W_{\lambda}$.

As in our previous work, we use the hyperfine structure (HFS) components from Prochaska et al. (2000) for the lines we use here of Sc II, Mn I, and Co I. For Ba II we adopt the HFS from McWilliam (1998). We use the laboratory spectroscopy of Lawler et al. (2001a, 2001b) to calculate the HFS patterns for La II and Eu II. We adopt the isotopic and HFS shifts for the $4057 \AA$ line of $\mathrm{Pb}$ I given by Van Eck et al. (2003); see her paper for references for the laboratory and theoretical atomic physics. McWilliam et al. (1995a) give the HFS pattern for the $\mathrm{Na} D$ lines. Although the difference between $\log \epsilon(\mathrm{Na})$ derived from the full HFS pattern and by just using two lines to represent the double is small, $<0.08$ dex, we use the full HFS pattern for these lines. A synthesis incorporating the list of hyperfine and isotopic components given by Hobbs et al. (1999) is used for the Li I resonance line for which an upper limit for its $W_{\lambda}$ was measured in one star. Spectral syntheses are carried out for each of the features with HFS to match the observed $W_{\lambda}$ and thus derive the abundance of the relevant species. For $\mathrm{Pb}$, because of the strong blending by $\mathrm{CH}$ features, the spectral synthesis used to determine the $\mathrm{Pb}$ abundance includes lines of ${ }^{12} \mathrm{CH},{ }^{13} \mathrm{CH}, \mathrm{Pb}$, and other metals.

Recall that the amount of $\mathrm{C}_{2}, \mathrm{CH}$, and $\mathrm{CN}$ formed is dependent on the amount of free carbon present (i.e., the amount not locked up in $\mathrm{CO}$ ), and that in general we do not have measure- ments of the $\mathrm{O}$ abundance in these EMP C stars. Thus, our derived $\mathrm{C}$ abundances are dependent on the choice made for the $\mathrm{O}$ abundance through molecular formation and equilibrium.

The abundance analysis is carried out using a current version of the LTE spectral synthesis program MOOG (Sneden 1973). We employ the grid of stellar atmospheres from Kurucz (1993) without convective overshoot, when available. We compute the abundances of the species observed in each star using the measured $W_{\lambda}$ values and the four stellar atmosphere models from this grid with the closest $T_{\text {eff }}$ and $\log g$ to each star's parameters. The abundances were interpolated using results from the closest stellar model atmospheres to the appropriate $T_{\text {eff }}$ and $\log g$ for each star given in Table 2.

Our HIRES spectra show that HE 0012-1441 is a doublelined spectroscopic binary. Since it is rather faint, spectra were taken on each of three consecutive nights with the intention of summing them to reach a high $\mathrm{S} / \mathrm{N}$. Comparison of the summed spectra for each of the three nights revealed the presence of double lines, as well as obvious differences in the velocity separation of the two components over the time span of $48 \mathrm{hr}$, as is shown in Figure 1 . The $v_{r}$ of the primary decreased by $6 \mathrm{~km} \mathrm{~s}^{-1}$ over that time span. The separation of the two components was largest on the last night, when it reached $28 \mathrm{~km} \mathrm{~s}^{-1}$. Thus, this binary must have a relatively short period and is probably similar to HE 00242523 (Cohen et al. 2002; Carretta et al. 2002; Lucatello et al. 2003). Only the sum of the three 1200 s HIRES exposures from

TABLE 5

Equivalent Widths for the Last Five Stars of the Primary Sample of C Stars from the HeS

\begin{tabular}{|c|c|c|c|c|c|c|c|c|}
\hline \multirow{2}{*}{$\begin{array}{c}\text { LiNe } \lambda \\
(\AA)\end{array}$} & \multirow[b]{2}{*}{ SPECIES } & \multirow{2}{*}{$\begin{array}{l}\text { EP } \\
(e V)\end{array}$} & \multirow{2}{*}{$\begin{array}{c}\log (g f) \\
(\operatorname{dex})\end{array}$} & \multicolumn{5}{|c|}{$W_{\lambda}(\mathrm{m} \AA)$} \\
\hline & & & & HE $1443+0113$ & HE $1509-0806$ & HE $2158-0348$ & HE $2232-0603$ & HE $2356-0410$ \\
\hline $4057.52 \ldots \ldots \ldots \ldots \ldots \ldots$ & $\mathrm{Mg}$ I & 4.34 & -1.200 & $\ldots$ & $\ldots$ & 29.0 & 72.0 & 18.0 \\
\hline $4167.28 \ldots \ldots \ldots \ldots \ldots \ldots$ & $\mathrm{Mg}_{\mathrm{I}}$ & 4.34 & -1.000 & $\ldots$ & $\ldots$ & $\ldots$ & 100.0 & $\ldots$ \\
\hline 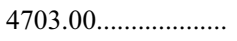 & $\mathrm{Mg}_{\mathrm{I}}$ & 4.34 & -0.670 & $\ldots$ & $\ldots$ & $\ldots$ & 105.4 & $\ldots$ \\
\hline $5172.70 \ldots \ldots \ldots \ldots \ldots \ldots \ldots$ & $\mathrm{Mg}_{\mathrm{I}}$ & 2.71 & -0.380 & 251.3 & 167.0 & 187.1 & 275.9 & 123.2 \\
\hline $5183.62 \ldots \ldots \ldots \ldots \ldots \ldots$ & Mg I & 2.72 & -0.160 & 333.8 & 211.0 & 237.5 & 356.6 & 141.9 \\
\hline 3961.52...................... & $\mathrm{Al}$ I & 0.00 & -0.340 & $\ldots$ & 119.0 & 129.1 & 140.0 & 111.3 \\
\hline $4435.69 \ldots \ldots \ldots \ldots \ldots \ldots$ & $\mathrm{Ca} \mathrm{I}$ & 1.89 & -0.520 & $\ldots$ & 29.0 & 56.0 & 60.3 & 26.7 \\
\hline $4454.79 \ldots \ldots \ldots \ldots \ldots \ldots$ & $\mathrm{Ca} \mathrm{I}$ & 1.90 & 0.260 & $\ldots$ & 74.0 & 94.0 & 95.1 & 79.6 \\
\hline $4670.41 \ldots \ldots \ldots \ldots \ldots \ldots$ & Sc II & 1.36 & -0.580 & $\ldots$ & $\ldots$ & $\ldots$ & 12.5 & 9.0 \\
\hline $3958.22 \ldots \ldots \ldots \ldots \ldots \ldots \ldots \ldots$ & Ti I & 0.05 & -0.160 & $\ldots$ & $\ldots$ & 50.0 & 62.3 & 21.0 \\
\hline
\end{tabular}

NoтEs.-Table 5 is published in its entirety in the electronic edition of the Astronomical Journal. A portion is shown here for guidance regarding its form and content. 
TABLE 6

Equivalent Widths for Redder Lines in the Spectra of Three C Stars

\begin{tabular}{|c|c|c|c|c|c|c|}
\hline \multirow{2}{*}{$\begin{array}{c}\text { LINE } \lambda \\
(\AA)\end{array}$} & \multirow[b]{2}{*}{ SPECIES } & \multirow{2}{*}{$\begin{array}{c}\text { EP } \\
(\mathrm{eV})\end{array}$} & \multirow{2}{*}{$\begin{array}{c}\log (g f) \\
\quad(\operatorname{dex})\end{array}$} & \multicolumn{3}{|c|}{$W_{\lambda}(\mathrm{m} \AA)$} \\
\hline & & & & HE $1410-0004$ & HE $1434-1442$ & HE $1443+0113$ \\
\hline 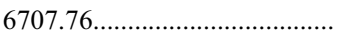 & $\mathrm{Li} \mathrm{I}$ & 0.00 & 0.178 & $<10.0$ & $\ldots$ & $\ldots$ \\
\hline 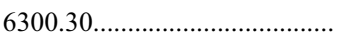 & $\mathrm{O}_{\mathrm{I}}$ & 0.00 & -9.78 & $<6.0$ & $\ldots$ & $\ldots$ \\
\hline 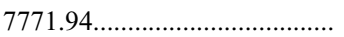 & $\mathrm{O}_{\mathrm{I}}$ & 9.15 & 0.369 & 7.0 & $\ldots$ & $\ldots$ \\
\hline 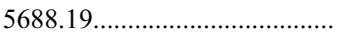 & $\mathrm{Na} I$ & 2.10 & -0.420 & 7.0 & $\ldots$ & $\ldots$ \\
\hline 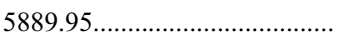 & $\mathrm{Na}$ I & 0.00 & 0.110 & 148.5 & 178.8 & 363.0 \\
\hline 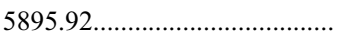 & $\mathrm{Na} I$ & 0.00 & -0.190 & 127.0 & 176.5 & 210.0 \\
\hline 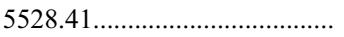 & Mg I & 4.34 & -0.480 & 29.5 & 71.8 & $\ldots$ \\
\hline 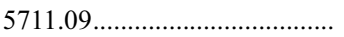 & Mg I & 4.34 & -0.167 & 6.0 & $\ldots$ & $\ldots$ \\
\hline 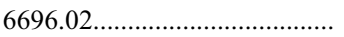 & Al I & 3.14 & -1.34 & 4.2 & $\ldots$ & $\ldots$ \\
\hline 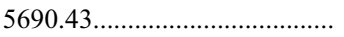 & Si I & 4.93 & -1.870 & $\ldots$ & 6.0 & $\ldots$ \\
\hline 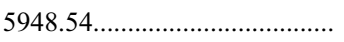 & Si I & 5.08 & -1.230 & $\ldots$ & 8.0 & $\ldots$ \\
\hline 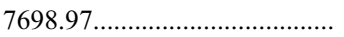 & $\mathrm{K}_{\mathrm{I}}$ & 0.00 & -0.168 & 12.6 & $\ldots$ & $\ldots$ \\
\hline $5588.75 \ldots \ldots \ldots$ & $\mathrm{Ca} \mathrm{I}$ & 2.52 & 0.437 & 11.1 & 36.3 & $\ldots$ \\
\hline 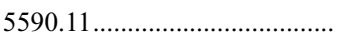 & $\mathrm{Ca} \mathrm{I}$ & 2.52 & -0.710 & $\ldots$ & 9.9 & $\ldots$ \\
\hline 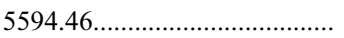 & $\mathrm{Ca} \mathrm{I}$ & 2.52 & -0.050 & 6.9 & 33.1 & $\ldots$ \\
\hline 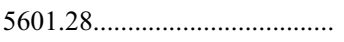 & $\mathrm{Ca} \mathrm{I}$ & 2.52 & -0.438 & $\ldots$ & 22.0 & $\cdots$ \\
\hline 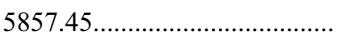 & $\mathrm{Ca} \mathrm{I}$ & 2.93 & 0.230 & $\ldots$ & 16.8 & $\ldots$ \\
\hline 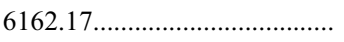 & $\mathrm{Ca} \mathrm{I}$ & 1.90 & -0.090 & 17.3 & $\ldots$ & $\ldots$ \\
\hline 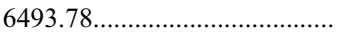 & $\mathrm{Ca} \mathrm{I}$ & 2.52 & 0.140 & 5.6 & $\ldots$ & $\ldots$ \\
\hline 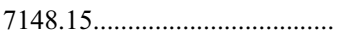 & $\mathrm{Ca} \mathrm{I}$ & 2.71 & 0.218 & 9.8 & $\ldots$ & $\ldots$ \\
\hline 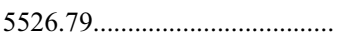 & Sc II & 1.77 & 0.130 & $\ldots$ & 17.5 & $\ldots$ \\
\hline 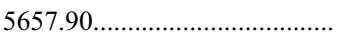 & Sc II & 1.51 & -0.500 & $\ldots$ & 11.7 & $\ldots$ \\
\hline 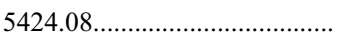 & Fe I & 4.32 & 0.510 & 35.2 & 52.6 & 88.0 \\
\hline 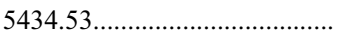 & $\mathrm{Fe}_{\mathrm{I}}$ & 1.01 & -2.130 & $\ldots$ & 75.4 & 107.0 \\
\hline 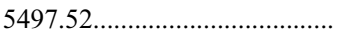 & $\mathrm{Fe} I$ & 1.01 & -2.830 & 10.4 & 27.2 & $\ldots$ \\
\hline 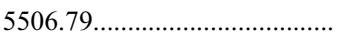 & $\mathrm{Fe}$ I & 0.99 & -2.790 & $\ldots$ & 35.7 & $\ldots$ \\
\hline 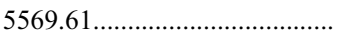 & $\mathrm{Fe}_{\mathrm{I}}$ & 3.42 & -0.486 & 9.1 & $\ldots$ & $\ldots$ \\
\hline 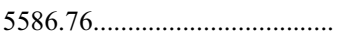 & Fe I & 3.37 & -0.140 & 10.4 & $\ldots$ & $\ldots$ \\
\hline 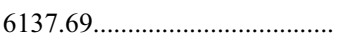 & Fe I & 2.59 & -1.350 & 11.2 & $\ldots$ & $\ldots$ \\
\hline 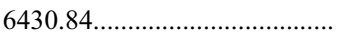 & $\mathrm{Fe}_{\mathrm{I}}$ & 2.18 & -1.950 & 6.9 & $\ldots$ & $\ldots$ \\
\hline 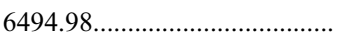 & $\mathrm{Fe}_{\mathrm{I}}$ & 2.40 & -1.240 & 13.0 & $\ldots$ & $\ldots$ \\
\hline 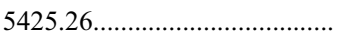 & $\mathrm{Fe}$ II & 3.00 & -3.240 & $\ldots$ & 11.0 & $\ldots$ \\
\hline 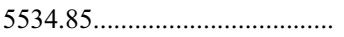 & $\mathrm{Fe}$ II & 3.25 & -2.640 & $\ldots$ & 6.3 & $\ldots$ \\
\hline 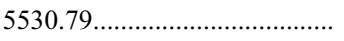 & Co I & 1.71 & -2.060 & $\ldots$ & $<9.0$ & $\ldots$ \\
\hline 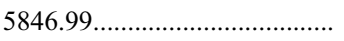 & $\mathrm{Ni} \mathrm{I}$ & 1.68 & -3.210 & $\ldots$ & $<4.0$ & $\ldots$ \\
\hline 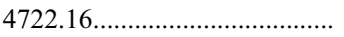 & $\mathrm{Zn} \mathrm{I}$ & 4.03 & -0.390 & $<8.0$ & $\ldots$ & $\ldots$ \\
\hline 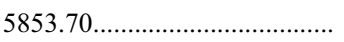 & $\mathrm{Ba}$ II & 0.60 & -1.010 & 19.0 & 73.5 & 121.0 \\
\hline 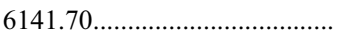 & Ba II & 0.70 & -0.070 & 61.1 & $\ldots$ & $\ldots$ \\
\hline 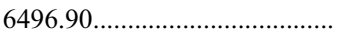 & $\mathrm{Ba}$ II & 0.60 & -0.380 & 57.4 & $\ldots$ & $\ldots$ \\
\hline 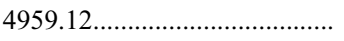 & $\mathrm{Nd}$ II & 0.06 & -0.800 & $\ldots$ & 32.0 & $\ldots$ \\
\hline 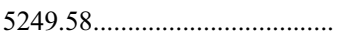 & $\mathrm{Nd}$ II & 0.98 & 0.200 & $\ldots$ & 16.5 & $\ldots$ \\
\hline 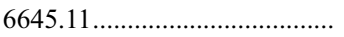 & $\mathrm{Eu} \mathrm{II}$ & 1.38 & 0.120 & $<6.0$ & $\ldots$ & $\ldots$ \\
\hline
\end{tabular}

the third night was used to determine $W_{\lambda}$; this night had the largest velocity separation, and hence was the easiest from which to measure the $W_{\lambda}$ of the primary component. This, of course, reduces the $\mathrm{S} / \mathrm{N}$ of the spectrum below that expected on the basis of the total integration time and below the desired value. We have assumed that the secondary, which contributes perhaps $\frac{1}{5}$ of the total $W_{\lambda}$ for selected lines, does not seriously affect the colors used to determine $T_{\text {eff }}$, which may not be a valid assumption. Furthermore, the lines from the secondary appear to be wider than those of the primary, suggesting a faint cool dwarf as the secondary star. (The secondary is too luminous to be a white dwarf with age $\sim 10$ Gyr.) For this star only, the $W_{\lambda}$ were not used; the $W_{\lambda}$ listed in Table 3 for this star are for guidance only. Instead the abundance was determined by matching the observed line profile for each spectral feature with the predicted one, varying $\epsilon(X)$. This ensured proper treatment of the partially blended lines due to the second component in the binary system. A luminosity ratio of 4 for the two components throughout the relevant wavelength range of the HIRES spectra was assumed to determine the $W_{\lambda}$ for this star.

The microturbulent velocity $\left(v_{t}\right)$ of a star can be determined spectroscopically by requiring the abundance to be independent of the strength of the lines. However, there are fewer usable Fe I lines in the complex spectra of these $\mathrm{C}$ stars than in stars with normal $\mathrm{C}$ and $\mathrm{N}$ and the same stellar parameters due to the rejection of large regions of the spectrum where the molecular features are strongest. Furthermore, the uncertainties in measurement of the remaining lines are larger, again due to possible molecular contamination and difficulties with continuum determination that do not occur in EMP stars with normal $\mathrm{C}$ and N. Based on our as yet unpublished analyses of a large sample of EMP giants from the HES, we set the $v_{t}$ to $1.6-1.8 \mathrm{~km} \mathrm{~s}^{-1}$, depending on $T_{\text {eff }}$. We checked in each case that a plot of derived Fe I abundance as a function of $W_{\lambda}$ looked reasonable but did not try to iterate on $v_{t}$ to achieve a perfectly constant Fe I abundance. 


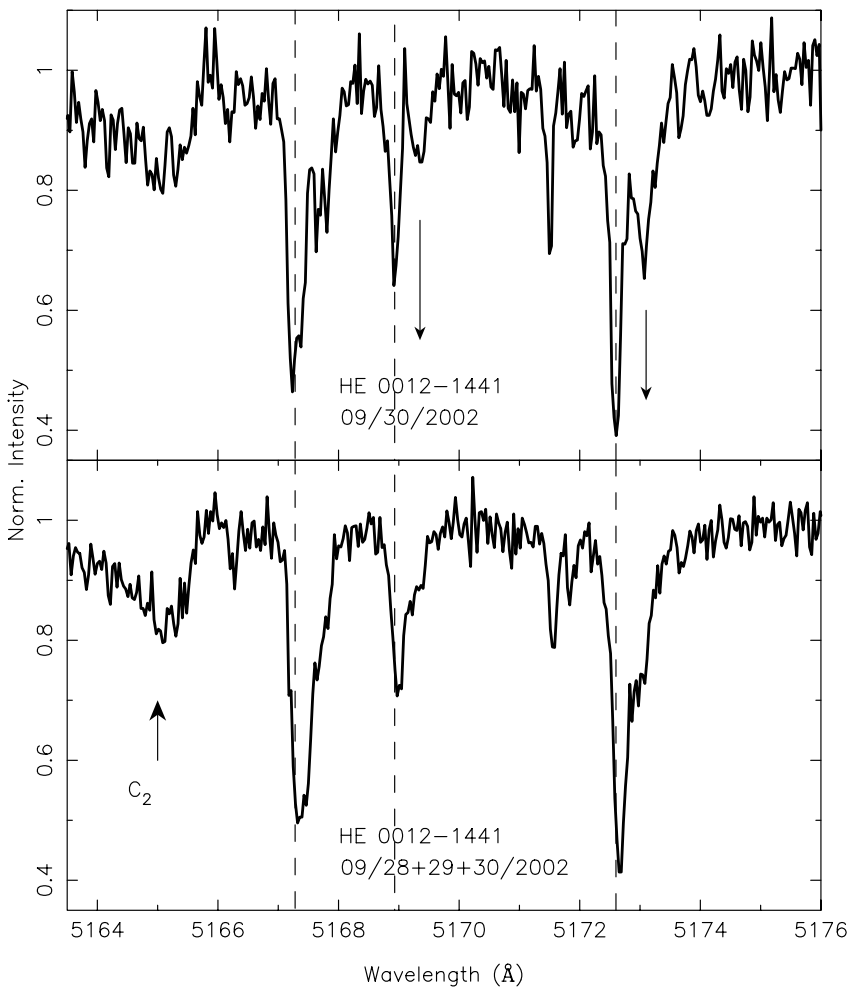

FIG. 1.-Top: Spectrum of the spectroscopic binary HE 0012-1441 from the night of 2002 September 30 in the region of the $\mathrm{Mg}$ triplet. The arrows indicate two lines from the secondary star. Bottom: Same as the top panel, but using the data summed over three nights from the 2002 September HIRES run. Note the difference in the line profiles of the Mg triplet lines (and some of the weaker lines as well). Note also that the lines from the secondary star are noticeably broader than those from the primary. The dashed vertical lines guide the eye to indicate the changing relative $v_{r}$ of the two components over a time span of $48 \mathrm{hr}$.

The abundances presented here could be improved. Spectral syntheses could be used for additional elements. A better determination of $T_{\text {eff }}$ and of $v_{t}$ could be attempted. However, Table 7 demonstrates that the results achieved here are reasonably good. This table gives the slope of a linear fit to the derived Fe I abundance from each observed line as a function of excitation potential (EP), $W_{\lambda} / \lambda$, and line wavelength. Assuming a perfect analysis, these slopes should all be zero. ${ }^{9}$

The full range in EP for the observed Fe I lines is only $3 \mathrm{eV}$. The mean slope for the derived Fe I abundance with EP for the $11 \mathrm{C}$ stars with entries in Table 7 is $+0.02 \mathrm{dex} \mathrm{eV}^{-1}$ with $\sigma=0.05 \mathrm{dex} \mathrm{eV}^{-1}$. We need to demonstrate that this mean and $\sigma$ are consistent with our known uncertainties. The value of $0.05 \mathrm{dex} \mathrm{eV}^{-1}$ found for $\sigma$ corresponds to a change in $T_{\mathrm{eff}}$ of $250 \mathrm{~K}$, somewhat larger than our adopted uncertainty in $T_{\text {eff }}$ discussed in $\S 2.1$ of $150 \mathrm{~K}$. However, the random component of the uncertainty in the $\mathrm{Fe}$ abundance derived from a single $\mathrm{Fe} \mathrm{I}$ line in a single star due primarily to errors in the $g f$-value assigned to the line is at least 0.2 dex. This leads to a $\sigma$ for the measured slopes of $\mathrm{Fe}$ I abundance versus EP beyond that expected purely from the adopted $T_{\text {eff }}$ uncertainty. To support this assertion we note that the correlation coefficients for the relationship within each star are low $(|r|<0.25$ in all cases).

\footnotetext{
${ }^{9}$ We ignore contributions from any issues that vary as a function of $T_{\text {eff }}$ that may not be included in our analysis, such as non-LTE effects, which might contribute to the measured slopes, and their rms dispersion. Large contributions to the $\sigma$ of the measured slopes from such terms can be excluded.
}

TABLE 7

Fitted Fe i Slopes with EP, Equivalent Width, and Wavelength

\begin{tabular}{|c|c|c|c|}
\hline ID & $\begin{array}{l}\Delta[\mathrm{X} / \mathrm{Fe}] / \Delta(\mathrm{EP})^{\mathrm{a}} \\
\quad\left(\mathrm{dex} \mathrm{eV}^{-1}\right)\end{array}$ & $\begin{array}{c}\Delta[\mathrm{X} / \mathrm{Fe}] / \Delta\left[W_{\lambda} / \lambda\right] \\
(\mathrm{dex})\end{array}$ & $\begin{array}{c}\Delta[\mathrm{X} / \mathrm{Fe}] / \Delta \lambda \\
\left(10^{-4} \operatorname{dex} \AA^{-1}\right)\end{array}$ \\
\hline HE $0012-1441^{\mathrm{b}} \ldots \ldots$ & $\ldots$ & $\ldots$ & \\
\hline HE $0058-0244 \ldots \ldots .$. & -0.013 & -0.198 & 1.02 \\
\hline HE $0143-0441 \ldots \ldots$. & -0.026 & 0.024 & 0.65 \\
\hline HE $0212-0557 \ldots \ldots$ & 0.088 & 0.207 & -5.33 \\
\hline HE $0336+0113 \ldots \ldots$ & 0.075 & -0.121 & 0.98 \\
\hline HE $1031-0020 \ldots \ldots$. & 0.039 & -0.117 & 2.02 \\
\hline HE $1150-0428 \ldots \ldots .$. & -0.066 & -0.027 & 0.14 \\
\hline HE $1410-0004 \ldots \ldots .$. & -0.014 & 0.001 & -0.08 \\
\hline HE $1410+0213^{c} \ldots \ldots$ & $\ldots$ & $\ldots$ & $\ldots$ \\
\hline HE $1434-1442 \ldots \ldots .$. & 0.056 & 0.006 & -1.38 \\
\hline HE $1443+0113^{\mathrm{d}} \ldots \ldots$ & $\ldots$ & $\ldots$ & $\ldots$ \\
\hline HE $1509-0806^{\mathrm{e}} \ldots .$. & $\ldots$ & $\ldots$ & $\ldots$ \\
\hline HE $2158-0348 \ldots \ldots .$. & 0.068 & -0.248 & 1.63 \\
\hline HE $2232-0603 \ldots \ldots$ & 0.057 & -0.013 & 1.87 \\
\hline HE $2356-0410 \ldots \ldots$. & -0.036 & 0.006 & -0.83 \\
\hline
\end{tabular}

a Typical range of EP is $3 \mathrm{eV}$.

b There were only 14 measured $\mathrm{Fe}$ I lines in this star

c There were only 12 detected $\mathrm{Fe}_{\mathrm{I}}$ lines in this star.

${ }^{d}$ There were only 10 measured $\mathrm{Fe}_{\mathrm{I}}$ lines in this star

e There were only 17 detected $\mathrm{Fe}_{\mathrm{I}}$ lines in this star.

The slopes for the Fe I abundance versus reduced equivalent width for the same set of 11 stars have a mean of -0.04 dex with $\sigma=0.12$ dex. The spread in this slope is completely consistent with our adopted uncertainty in $v_{t}$ of $0.2 \mathrm{~km} \mathrm{~s}^{-1}$. The set of correlation coefficients are low $(|r|<0.35$ in all cases $)$ here also.

The results for the abundances of typically 20 species in each star (only nine in HE 1410+0213 and only five for HE 1443+ $0113)$ are given in Tables $8-11$. We tabulate both $\log \epsilon(X)$ and $[\mathrm{X} / \mathrm{Fe}]$; our adopted solar abundances can be inferred directly from these tables. The ${ }^{12} \mathrm{C} /{ }^{13} \mathrm{C}$ ratios determined from the $\mathrm{CH}$ and the $\mathrm{C}_{2}$ bands are given in Table 12 .

Table 13 gives the changes in the deduced abundances for small changes in $T_{\text {eff }}, \log g, v_{t}$, and $W_{\lambda}$ in the $[\mathrm{Fe} / \mathrm{H}]$ of the model atmosphere used for an EMP giant with $T_{\text {eff }} \sim 5200 \mathrm{~K}$. The last column gives expected random uncertainties for $[\mathrm{X} / \mathrm{Fe}]$ appropriate for a single star, combining in quadrature the uncertainties in $[\mathrm{X} / \mathrm{Fe}]$ resulting from the errors in stellar parameters established in $\S 2.1$, i.e., an uncertainty of $\pm 150 \mathrm{~K}$ in $T_{\text {eff }}$, of \pm 0.4 dex in $\log g$, of $\pm 0.5 \mathrm{dex}$ in the metallicity assumed in the model atmosphere used for the analysis, of $\pm 0.2 \mathrm{~km} \mathrm{~s}^{-1}$ for $v_{t}$, and of a contribution representing the errors in the measured equivalent widths. This last term is set at $20 \%$ (approximately equivalent to 0.08 dex abundance uncertainty but depending on line strength) for a single detected line (which may be an underestimate for the complex spectra of the $\mathrm{C}$ stars) and is scaled based on the number of detected lines. The contribution of the various terms, particularly that of $\log g$, which will be smaller for hotter stars, may vary somewhat with $T_{\text {eff }}$. Systematic uncertainties, such as might arise from errors in the scale of the transition probabilities for an element, are not included in the entries in Table 13. Random errors in the $g f$-value for a particular line are not relevant to this calculation provided that the same line list is used throughout.

\subsection{The ${ }^{12} \mathrm{C} /{ }^{13} \mathrm{C}$ Ratios}

We have measured the isotopic ratio ${ }^{12} \mathrm{C} /{ }^{13} \mathrm{C}$ for the $\mathrm{C}$ stars from our sample with the highest $\mathrm{S} / \mathrm{N}$ spectra using the line list for the $4300 \AA$ region of the $G$ band of $\mathrm{CH}$ as described in Cohen et al. (2003). Spectral syntheses of the features of ${ }^{13} \mathrm{CH}$ at 4211.3 , 
TABLE 8

Abundances for the First Four EMP C Stars from the HES

\begin{tabular}{|c|c|c|c|c|c|c|c|c|c|c|c|c|c|c|c|c|}
\hline \multirow[b]{2}{*}{ Species } & \multicolumn{4}{|c|}{ HE $0012-1441$} & \multicolumn{4}{|c|}{ HE $0058-0244$} & \multicolumn{4}{|c|}{ HE $0143-0441$} & \multicolumn{4}{|c|}{ HE $0212-0557$} \\
\hline & $\begin{array}{c}{[\mathrm{X} / \mathrm{Fe}]} \\
(\mathrm{dex})\end{array}$ & $\begin{array}{l}\log \epsilon(X) \\
\quad(\operatorname{dex})\end{array}$ & No. Lines & $\begin{array}{c}\sigma \\
(\operatorname{dex})\end{array}$ & $\begin{array}{c}{[\mathrm{X} / \mathrm{Fe}]} \\
(\mathrm{dex})\end{array}$ & $\begin{array}{c}\log \epsilon(\mathrm{X}) \\
\quad(\mathrm{dex})\end{array}$ & $\begin{array}{c}\text { No. } \\
\text { Lines }\end{array}$ & $\begin{array}{c}\sigma \\
(\operatorname{dex})\end{array}$ & $\begin{array}{c}{[\mathrm{X} / \mathrm{Fe}]} \\
(\mathrm{dex})\end{array}$ & $\begin{array}{c}\log \epsilon(X) \\
\quad(\operatorname{dex})\end{array}$ & $\begin{array}{c}\text { No. } \\
\text { Lines }\end{array}$ & $\begin{array}{c}\sigma \\
(\operatorname{dex})\end{array}$ & $\begin{array}{c}{[\mathrm{X} / \mathrm{Fe}]} \\
(\mathrm{dex})\end{array}$ & $\begin{array}{c}\log \epsilon(X) \\
\quad(\operatorname{dex})\end{array}$ & $\begin{array}{c}\text { No. } \\
\text { Lines }\end{array}$ & $\begin{array}{c}\sigma \\
(\operatorname{dex})\end{array}$ \\
\hline $\mathrm{C}(\mathrm{CH}) \ldots \ldots \ldots$ & 1.59 & 7.66 & 1 & $\ldots$ & 1.92 & 7.76 & 1 & $\ldots$ & 1.98 & 8.26 & 1 & $\ldots$ & 1.74 & 8.06 & 1 & $\ldots$ \\
\hline $\mathrm{N}(\mathrm{CN}) \ldots \ldots \ldots$ & 0.64 & 6.05 & 1 & $\ldots$ & 1.77 & 6.95 & 1 & $\ldots$ & $1.73^{\mathrm{a}}$ & $7.35^{\mathrm{a}}$ & 1 & $\ldots$ & 1.09 & 6.75 & 1 & $\ldots$ \\
\hline Mg І.............. & 0.91 & 5.93 & 4 & 0.16 & 0.54 & 5.33 & 3 & 0.19 & 0.63 & 5.86 & 3 & 0.08 & 0.04 & 5.32 & 1 & $\ldots$ \\
\hline Al I ............... & $\ldots$ & $\ldots$ & $\ldots$ & $\ldots$ & 0.34 & 4.06 & 1 & $\ldots$ & -0.22 & 3.94 & 1 & $\ldots$ & 0.01 & 4.21 & 1 & $\ldots$ \\
\hline $\mathrm{Ca}$ I.................. & 0.42 & 4.26 & 3 & 0.11 & 0.96 & 4.57 & 2 & 0.20 & 0.43 & 4.48 & 2 & 0.17 & 0.14 & 4.23 & 1 & $\ldots$ \\
\hline Sc II .............. & $\ldots$ & $\ldots$ & $\ldots$ & $\ldots$ & 0.56 & 0.91 & 1 & $\ldots$ & 0.67 & 1.46 & 1 & $\ldots$ & $\ldots$ & $\ldots$ & $\ldots$ & $\ldots$ \\
\hline Ti г............... & 0.03 & 2.50 & 2 & 0.10 & 0.57 & 2.81 & 10 & 0.17 & 0.54 & 3.22 & 5 & 0.26 & 0.14 & 2.87 & 5 & 0.32 \\
\hline Ti II ............... & 0.13 & 2.60 & 5 & 0.08 & 0.55 & 2.79 & 10 & 0.16 & 0.26 & 2.94 & 8 & 0.16 & 0.32 & 3.04 & 8 & 0.32 \\
\hline 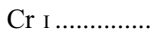 & -0.18 & 2.97 & 1 & $\ldots$ & -0.35 & 2.57 & 1 & $\ldots$ & -0.38 & 2.98 & 1 & $\ldots$ & -0.09 & 3.31 & 2 & 0.03 \\
\hline Mn I.............. & $\ldots$ & $\ldots$ & $\ldots$ & $\ldots$ & -0.39 & 2.24 & 3 & 0.11 & -0.72 & 2.36 & 2 & 0.23 & -0.55 & 2.57 & 4 & 0.46 \\
\hline Fe I ............. & $-2.52^{b}$ & 4.93 & 14 & 0.16 & $-2.75^{b}$ & 4.70 & 35 & 0.21 & $-2.31^{\mathrm{b}}$ & 5.14 & 31 & 0.18 & $-2.27^{b}$ & 5.18 & 17 & 0.26 \\
\hline Fe II ............. & -0.15 & 4.78 & 5 & 0.19 & 0.02 & 4.72 & 7 & 0.12 & -0.22 & 4.92 & 5 & 0.11 & 0.04 & 5.23 & 7 & 0.28 \\
\hline Co I.................... & $\ldots$ & $\ldots$ & $\ldots$ & $\ldots$ & 0.17 & 2.34 & 1 & $\ldots$ & 0.47 & 3.08 & 1 & $\ldots$ & $\ldots$ & $\ldots$ & $\ldots$ & $\ldots$ \\
\hline Ni I .............. & $\ldots$ & $\cdots$ & $\ldots$ & $\ldots$ & -0.33 & 3.17 & 1 & $\ldots$ & -0.31 & 3.63 & 1 & $\ldots$ & $\ldots$ & $\ldots$ & $\ldots$ & $\ldots$ \\
\hline Zn I................ & $\ldots$ & $\ldots$ & $\ldots$ & $\ldots$ & $\ldots$ & $\ldots$ & $\ldots$ & $\ldots$ & 0.46 & 2.76 & 1 & $\ldots$ & $\ldots$ & $\ldots$ & $\ldots$ & $\ldots$ \\
\hline Sr II................. & $\ldots$ & $\ldots$ & $\ldots$ & $\ldots$ & 0.34 & 0.49 & 1 & $\ldots$ & 0.86 & 1.45 & 1 & $\ldots$ & -0.05 & 0.59 & 1 & $\ldots$ \\
\hline Y II .............. & $\ldots$ & $\ldots$ & $\ldots$ & $\ldots$ & 0.52 & 0.01 & 2 & 0.03 & 0.59 & 0.52 & 2 & 0.21 & 0.55 & 0.53 & 3 & 0.16 \\
\hline $\mathrm{Zr}$ II............... & $\ldots$ & $\ldots$ & $\ldots$ & $\ldots$ & $\ldots$ & $\ldots$ & $\ldots$ & $\ldots$ & 1.05 & 1.34 & 1 & $\ldots$ & $\ldots$ & $\ldots$ & $\ldots$ & $\ldots$ \\
\hline Ba II ............... & 1.15 & 0.76 & 2 & 0.27 & 2.04 & 1.42 & 3 & 0.16 & 2.32 & 2.14 & 3 & 0.12 & 2.18 & 2.04 & 2 & 0.06 \\
\hline La II ............... & $\ldots$ & $\ldots$ & $\ldots$ & $\ldots$ & 1.70 & 0.09 & 4 & 0.04 & 1.78 & 0.61 & 4 & 0.15 & 2.28 & 1.15 & 3 & 0.22 \\
\hline Ce II.............. & $\ldots$ & $\ldots$ & $\ldots$ & $\ldots$ & 1.88 & 0.68 & 5 & 0.22 & 1.93 & 1.17 & 3 & 0.21 & 2.14 & 1.42 & 1 & $\ldots$ \\
\hline $\operatorname{Pr}$ II................. & $\ldots$ & $\ldots$ & $\ldots$ & $\ldots$ & $\ldots$ & $\ldots$ & $\ldots$ & $\ldots$ & $\ldots$ & $\ldots$ & $\ldots$ & $\ldots$ & 2.37 & 0.81 & 1 & $\ldots$ \\
\hline $\mathrm{Nd}$ II ............. & $\ldots$ & $\ldots$ & $\ldots$ & $\ldots$ & 1.91 & 0.66 & 4 & 0.24 & 2.17 & 1.37 & 3 & 0.21 & 1.90 & 1.13 & 1 & $\ldots$ \\
\hline Eu II .............. & $\ldots$ & $\ldots$ & $\ldots$ & $\ldots$ & 1.70 & -0.54 & 3 & 0.14 & 1.46 & -0.34 & 3 & 0.17 & $\ldots$ & $\ldots$ & $\ldots$ & $\ldots$ \\
\hline $\mathrm{Pb}$ I .................. & $<1.92$ & $<1.35$ & 1 & $\ldots$ & 2.79 & 1.99 & 1 & $\ldots$ & 3.11 & 2.75 & 1 & $\ldots$ & $\mathrm{c}$ & $\ldots$ & $\ldots$ & $\ldots$ \\
\hline
\end{tabular}

a This value supersedes that given in Cohen et al. (2004).

b This is $[\mathrm{Fe} / \mathrm{H}]$.

c $\mathrm{No} \mathrm{Pb}$ abundance, as the large negative $v_{r}$ moves the $\mathrm{Pb}$ I $\lambda 4057$ line onto a CCD defect.

TABLE 9

Abundances for the Second Four EMP C Stars from the HES

\begin{tabular}{|c|c|c|c|c|c|c|c|c|c|c|c|c|c|c|c|c|}
\hline \multirow[b]{2}{*}{ SPECIES } & \multicolumn{4}{|c|}{ HE $0336+0113$} & \multicolumn{4}{|c|}{ HE $1031-0020$} & \multicolumn{4}{|c|}{ HE $1150-0428$} & \multicolumn{4}{|c|}{ HE $1410+0213$} \\
\hline & $\begin{array}{c}{[\mathrm{X} / \mathrm{Fe}]} \\
(\mathrm{dex})\end{array}$ & $\begin{array}{c}\log \epsilon(X) \\
(\operatorname{dex})\end{array}$ & $\begin{array}{l}\text { No. } \\
\text { Lines }\end{array}$ & $\begin{array}{c}\sigma \\
(\mathrm{dex})\end{array}$ & $\begin{array}{c}{[\mathrm{X} / \mathrm{Fe}]} \\
(\mathrm{dex})\end{array}$ & $\begin{array}{c}\log \epsilon(X) \\
\quad(\operatorname{dex})\end{array}$ & $\begin{array}{l}\text { No. } \\
\text { Lines }\end{array}$ & $\begin{array}{c}\sigma \\
(\mathrm{dex})\end{array}$ & $\begin{array}{c}{[\mathrm{X} / \mathrm{Fe}]} \\
(\mathrm{dex})\end{array}$ & $\begin{array}{c}\log \epsilon(X) \\
(\operatorname{dex})\end{array}$ & $\begin{array}{c}\text { No. } \\
\text { Lines }\end{array}$ & $\begin{array}{c}\sigma \\
(\operatorname{dex})\end{array}$ & $\begin{array}{c}{[\mathrm{X} / \mathrm{Fe}]} \\
(\mathrm{dex})\end{array}$ & $\begin{array}{c}\log \epsilon(X) \\
(\operatorname{dex})\end{array}$ & $\begin{array}{c}\text { No. } \\
\text { Lines }\end{array}$ & $\begin{array}{c}\sigma \\
(\mathrm{dex})\end{array}$ \\
\hline $\mathrm{C}(\mathrm{CH}) \ldots \ldots \ldots$ & 2.25 & 8.16 & 1 & $\ldots$ & 1.63 & 7.36 & 1 & $\ldots$ & 2.37 & 7.66 & 1 & $\ldots$ & 1.73 & 8.16 & 1 & $\ldots$ \\
\hline $\mathrm{N}(\mathrm{CN}) \ldots \ldots \ldots$ & 1.60 & 6.85 & 1 & $\ldots$ & 2.48 & 7.55 & 1 & $\ldots$ & 2.52 & 7.15 & 1 & $\ldots$ & 1.78 & 7.55 & 1 & $\ldots$ \\
\hline Na I.............. & $\ldots$ & $\ldots$ & $\ldots$ & $\ldots$ & $\ldots$ & $\ldots$ & $\ldots$ & $\ldots$ & $\ldots$ & $\ldots$ & $\ldots$ & $\ldots$ & $\ldots$ & $\ldots$ & $\ldots$ & $\ldots$ \\
\hline Mg I............ & 1.04 & 5.90 & 3 & 0.18 & 0.50 & 5.19 & 3 & 0.18 & 0.34 & 4.58 & 2 & 0.06 & 0.18 & 5.56 & 2 & 0.03 \\
\hline $\mathrm{Al}$ I .................. & 0.34 & 4.13 & 1 & $\ldots$ & 0.88 & 4.49 & 1 & $\ldots$ & $\ldots$ & $\ldots$ & $\ldots$ & $\ldots$ & $\ldots$ & $\ldots$ & $\ldots$ & $\ldots$ \\
\hline Si I .................. & $\ldots$ & $\ldots$ & $\ldots$ & $\ldots$ & $\ldots$ & $\ldots$ & $\ldots$ & $\ldots$ & $\ldots$ & $\ldots$ & $\ldots$ & $\ldots$ & $\ldots$ & $\ldots$ & $\ldots$ & $\ldots$ \\
\hline $\mathrm{Ca} \mathrm{I} \ldots \ldots \ldots \ldots . . . .$. & 0.42 & 4.10 & 1 & $\ldots$ & 1.12 & 4.62 & 3 & 0.22 & 1.03 & 4.09 & 3 & 0.04 & $\ldots$ & $\ldots$ & $\ldots$ & $\ldots$ \\
\hline Ti г.............. & 0.39 & 2.70 & 5 & 0.19 & 0.46 & 2.60 & 11 & 0.31 & 0.49 & 2.17 & 5 & 0.12 & 0.29 & 3.12 & 2 & 0.04 \\
\hline Ti II ............... & 0.11 & 2.42 & 7 & 0.13 & 0.80 & 2.93 & 15 & 0.37 & 0.50 & 2.19 & 7 & 0.45 & 0.36 & 3.19 & 1 & $\ldots$ \\
\hline $\mathrm{Cr}$ I ................ & -0.26 & 2.73 & 1 & $\ldots$ & -0.38 & 2.43 & 1 & $\ldots$ & -0.70 & 1.67 & 1 & $\ldots$ & -0.55 & 2.96 & 1 & $\ldots$ \\
\hline Mn I............. & -0.21 & 2.50 & 2 & 0.14 & 0.03 & 2.56 & 5 & 0.45 & $\ldots$ & $\ldots$ & $\ldots$ & $\ldots$ & $\ldots$ & $\ldots$ & $\ldots$ & $\ldots$ \\
\hline Fe I ................ & $-2.68^{\mathrm{a}}$ & 4.77 & 31 & 0.22 & $-2.86^{\mathrm{a}}$ & 4.59 & 33 & 0.30 & $-3.30^{\mathrm{a}}$ & 4.15 & 23 & 0.26 & $-2.16^{\mathrm{a}} 0.00$ & 5.29 & 12 & 0.31 \\
\hline Fe II ............. & -0.23 & 4.55 & 4 & 0.19 & -0.05 & 4.54 & 8 & 0.32 & 0.00 & 4.15 & 4 & 0.09 & -0.41 & 4.88 & 3 & 0.21 \\
\hline Co I................. & 0.15 & 2.40 & 1 & $\ldots$ & $\ldots$ & $\ldots$ & $\ldots$ & $\ldots$ & $\ldots$ & $\ldots$ & $\ldots$ & $\ldots$ & $\ldots$ & $\ldots$ & $\ldots$ & $\ldots$ \\
\hline $\mathrm{Zn}$ I................. & $\ldots$ & $\ldots$ & $\ldots$ & $\ldots$ & $\ldots$ & $\ldots$ & $\ldots$ & $\ldots$ & $\ldots$ & $\ldots$ & $\ldots$ & $\ldots$ & $\ldots$ & $\ldots$ & $\ldots$ & $\ldots$ \\
\hline Sr II................ & 1.68 & 1.91 & 1 & $\ldots$ & 0.31 & 0.35 & 1 & $\ldots$ & -0.39 & -0.80 & 1 & $\ldots$ & $\ldots$ & $\ldots$ & $\ldots$ & $\ldots$ \\
\hline Y II ................. & 1.40 & 0.96 & 2 & 0.29 & 0.25 & -0.37 & 2 & 0.02 & $<0.23$ & $<-0.83$ & 1 & $\ldots$ & $\ldots$ & $\ldots$ & $\ldots$ & $\ldots$ \\
\hline Ba II ............. & 2.63 & 2.08 & 3 & 0.32 & 1.21 & 0.48 & 2 & 0.24 & -0.61 & -1.78 & 2 & 0.14 & 0.07 & 0.04 & 1 & $\ldots$ \\
\hline La II ............... & 1.93 & 0.39 & 4 & 0.14 & 1.16 & -0.56 & 4 & 0.15 & $<1.16$ & $<-1.00$ & 3 & 0.25 & $\ldots$ & $\ldots$ & $\ldots$ & $\ldots$ \\
\hline Ce II............. & 2.30 & 1.17 & 6 & 0.18 & 1.40 & 0.09 & 1 & $\ldots$ & $<2.04$ & $<0.29$ & 3 & 0.65 & $\ldots$ & $\ldots$ & $\ldots$ & $\ldots$ \\
\hline Nd II ............ & 2.12 & 0.94 & 6 & 0.27 & 1.72 & 0.36 & 4 & 0.11 & $<1.59$ & $<-0.21$ & 2 & 0.41 & $\ldots$ & $\ldots$ & $\ldots$ & $\ldots$ \\
\hline Eu II ............... & 1.18 & -0.99 & 2 & 0.13 & $<0.87$ & $<-1.48$ & 1 & $\ldots$ & $<1.45$ & $<-1.34$ & 1 & $\ldots$ & $\ldots$ & $\ldots$ & $\ldots$ & $\ldots$ \\
\hline $\mathrm{Pb}$ I .................. & $<2.28$ & $<1.55$ & 1 & $\ldots$ & 2.66 & 1.75 & 1 & $\ldots$ & $\ldots$ & $\ldots$ & $\ldots$ & $\ldots$ & $\mathrm{b}$ & $\ldots$ & $\ldots$ & $\ldots$ \\
\hline
\end{tabular}

a This is $[\mathrm{Fe} / \mathrm{H}]$.

b The $\mathrm{Pb}$ I $\lambda 4057$ line is swamped by molecular features. 
TABLE 10

Abundances for the Third Four EMP C Stars from the HES

\begin{tabular}{|c|c|c|c|c|c|c|c|c|c|c|c|c|c|c|c|c|}
\hline \multirow[b]{2}{*}{ SPECIES } & \multicolumn{4}{|c|}{ HE $1410-0004$} & \multicolumn{4}{|c|}{ HE $1434-1442$} & \multicolumn{4}{|c|}{ HE $1443+0113$} & \multicolumn{4}{|c|}{ HE 1509-0806 } \\
\hline & $\begin{array}{c}{[\mathrm{X} / \mathrm{Fe}]} \\
(\mathrm{dex})\end{array}$ & $\begin{array}{c}\log \epsilon(X) \\
\quad(\operatorname{dex})\end{array}$ & $\begin{array}{c}\text { No. } \\
\text { Lines }\end{array}$ & $\begin{array}{c}\sigma \\
(\mathrm{dex})\end{array}$ & $\begin{array}{c}{[\mathrm{X} / \mathrm{Fe}]} \\
(\mathrm{dex})\end{array}$ & $\begin{array}{l}\log \epsilon(X) \\
\quad(\operatorname{dex})\end{array}$ & $\begin{array}{c}\text { No. } \\
\text { Lines }\end{array}$ & $\begin{array}{c}\sigma \\
(\mathrm{dex})\end{array}$ & $\begin{array}{c}{[\mathrm{X} / \mathrm{Fe}]} \\
(\mathrm{dex})\end{array}$ & $\begin{array}{c}\log \epsilon(X) \\
\quad(\operatorname{dex})\end{array}$ & $\begin{array}{c}\text { No. } \\
\text { Lines }\end{array}$ & $\begin{array}{c}\sigma \\
(\operatorname{dex})\end{array}$ & $\begin{array}{c}{[\mathrm{X} / \mathrm{Fe}]} \\
(\mathrm{dex})\end{array}$ & $\begin{array}{l}\log \epsilon(X) \\
\quad(\operatorname{dex})\end{array}$ & $\begin{array}{c}\text { No. } \\
\text { Lines }\end{array}$ & $\begin{array}{c}\sigma \\
(\mathrm{dex})\end{array}$ \\
\hline Li I....................... & $<1.03^{\mathrm{a}}$ & $<1.32$ & 1 & $\ldots$ & $\ldots$ & $\ldots$ & $\ldots$ & $\ldots$ & $\ldots$ & $\ldots$ & $\ldots$ & $\ldots$ & $\ldots$ & $\ldots$ & $\ldots$ & $\ldots$ \\
\hline $\mathrm{C}(\mathrm{CH}) \ldots \ldots \ldots \ldots .$. & 1.99 & 7.56 & 1 & $\ldots$ & 1.95 & 8.16 & 1 & $\ldots$ & 1.84 & 8.36 & 1 & $\ldots$ & 1.98 & 7.66 & 1 & $\ldots$ \\
\hline $\mathrm{N}(\mathrm{CN}) \ldots \ldots \ldots \ldots$ & $\ldots$ & $\ldots$ & $\ldots$ & $\ldots$ & 1.40 & 6.95 & 1 & $\ldots$ & $\ldots$ & $\ldots$ & $\ldots$ & $\ldots$ & 2.23 & 7.25 & 1 & $\ldots$ \\
\hline 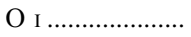 & 1.18 & 6.90 & 1 & $\ldots$ & $\ldots$ & $\ldots$ & $\ldots$ & $\ldots$ & $\ldots$ & $\ldots$ & $\ldots$ & $\ldots$ & $\ldots$ & $\ldots$ & $\ldots$ & $\ldots$ \\
\hline Na I.................. & 0.48 & 3.78 & 3 & 0.20 & 0.03 & 3.97 & 4 & 0.23 & 0.37 & 4.62 & 2 & 0.27 & $\ldots$ & $\ldots$ & $\ldots$ & $\ldots$ \\
\hline Mg I.................. & 0.58 & 5.10 & 6 & 0.29 & 0.30 & 5.45 & 3 & 0.05 & 0.37 & 5.84 & 2 & 0.09 & 0.64 & 5.27 & 2 & 0.11 \\
\hline Al I ..................... & $\ldots$ & $\ldots$ & $\ldots$ & $\ldots$ & $\ldots$ & $\ldots$ & $\ldots$ & $\ldots$ & $\ldots$ & $\ldots$ & $\ldots$ & $\ldots$ & 0.28 & 3.84 & 1 & $\ldots$ \\
\hline 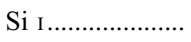 & $\ldots$ & $\ldots$ & $\ldots$ & $\ldots$ & 0.74 & 5.91 & 2 & 0.26 & $\ldots$ & $\ldots$ & $\ldots$ & $\ldots$ & $\ldots$ & $\ldots$ & $\ldots$ & $\ldots$ \\
\hline $\mathrm{K}_{\mathrm{I}} \ldots \ldots \ldots \ldots \ldots \ldots \ldots$ & 0.71 & 2.81 & 1 & $\ldots$ & $\ldots$ & $\ldots$ & $\ldots$ & $\ldots$ & $\ldots$ & $\ldots$ & $\cdots$ & $\ldots$ & $\ldots$ & $\ldots$ & $\ldots$ & $\ldots$ \\
\hline $\mathrm{Ca}$ I..................... & 0.11 & 3.45 & 5 & 0.16 & 0.24 & 4.21 & 5 & 0.23 & $\ldots$ & $\ldots$ & $\ldots$ & $\ldots$ & 0.55 & 4.00 & 2 & 0.06 \\
\hline Sc II ................. & $\ldots$ & $\ldots$ & $\ldots$ & $\ldots$ & 0.18 & 0.90 & 2 & 0.10 & $\ldots$ & $\ldots$ & $\ldots$ & $\ldots$ & $\ldots$ & $\ldots$ & $\ldots$ & $\ldots$ \\
\hline Ti I ...................... & 0.38 & 2.35 & 2 & 0.24 & 0.31 & 2.91 & 5 & 0.19 & $\ldots$ & $\ldots$ & $\ldots$ & $\ldots$ & 0.28 & 2.36 & 5 & 0.08 \\
\hline Ti II .................. & 0.22 & 2.20 & 4 & 0.09 & 0.68 & 3.29 & 8 & 0.32 & $\ldots$ & $\ldots$ & $\ldots$ & $\ldots$ & 0.96 & 3.04 & 11 & 0.28 \\
\hline Cr I .................... & -0.19 & 2.47 & 2 & 0.09 & -0.30 & 2.99 & 2 & 0.19 & $\ldots$ & $\ldots$ & $\ldots$ & $\ldots$ & -0.08 & 2.68 & 1 & $\ldots$ \\
\hline Mn I................... & -0.61 & 1.76 & 2 & 0.21 & -0.40 & 2.60 & 3 & 0.19 & $\ldots$ & $\ldots$ & $\ldots$ & $\ldots$ & -0.05 & 2.43 & 3 & 0.42 \\
\hline 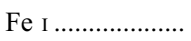 & $-3.02^{\mathrm{b}}$ & 4.43 & 22 & 0.17 & $-2.39^{\mathrm{b}}$ & 5.07 & 20 & 0.18 & $-2.07^{\mathrm{b}}$ & 5.38 & 10 & 0.21 & $-2.91^{\mathrm{b}}$ & 4.54 & 18 & 0.16 \\
\hline Fe II .................... & 0.02 & 4.45 & 5 & 0.09 & 0.03 & 5.09 & 8 & 0.23 & $\ldots$ & $\ldots$ & $\ldots$ & $\ldots$ & 0.17 & 4.71 & 5 & 0.24 \\
\hline Co I....................... & $\ldots$ & $\ldots$ & $\ldots$ & $\ldots$ & $<1.53$ & $<4.07$ & 1 & $\ldots$ & $\ldots$ & $\ldots$ & $\ldots$ & $\ldots$ & 0.10 & 2.11 & 1 & $\ldots$ \\
\hline Ni I ................... & $\ldots$ & $\ldots$ & $\ldots$ & $\ldots$ & 0.76 & 4.63 & 1 & $\ldots$ & $\ldots$ & $\cdots$ & $\cdots$ & $\ldots$ & $\ldots$ & $\ldots$ & $\ldots$ & $\cdots$ \\
\hline Zn I..................... & $<0.90$ & $<2.48$ & 1 & $\ldots$ & $\ldots$ & $\ldots$ & $\ldots$ & $\ldots$ & $\ldots$ & $\ldots$ & $\ldots$ & $\ldots$ & $\ldots$ & $\ldots$ & $\ldots$ & $\ldots$ \\
\hline 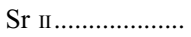 & 0.18 & 0.06 & 1 & $\ldots$ & $\ldots$ & $\ldots$ & $\ldots$ & $\ldots$ & $\ldots$ & $\ldots$ & $\ldots$ & $\ldots$ & 1.12 & 1.11 & 1 & $\ldots$ \\
\hline Y II .................. & $\ldots$ & $\ldots$ & $\ldots$ & $\ldots$ & 0.37 & 0.23 & 2 & 0.03 & $\ldots$ & $\ldots$ & $\ldots$ & $\ldots$ & 0.95 & 0.28 & 2 & 0.16 \\
\hline Ва II.................... & 1.06 & 0.17 & 5 & 0.24 & 1.23 & 0.98 & 3 & 0.17 & 1.40 & 1.46 & 2 & 0.04 & 1.93 & 1.15 & 2 & 0.18 \\
\hline La II ................... & $\ldots$ & $\ldots$ & $\ldots$ & $\ldots$ & $\ldots$ & $\ldots$ & $\ldots$ & $\ldots$ & $\ldots$ & $\ldots$ & $\ldots$ & $\ldots$ & 1.67 & -0.10 & 4 & 0.16 \\
\hline Ce & $\ldots$ & $\ldots$ & $\ldots$ & $\ldots$ & $\ldots$ & $\ldots$ & $\ldots$ & $\ldots$ & $\ldots$ & $\ldots$ & $\ldots$ & $\ldots$ & 1.89 & 0.53 & 3 & 0.38 \\
\hline Nd II ................... & $\ldots$ & $\ldots$ & $\ldots$ & $\ldots$ & 1.70 & 0.81 & 2 & 0.31 & $\ldots$ & $\ldots$ & $\ldots$ & $\ldots$ & 2.18 & 0.77 & 4 & 0.70 \\
\hline Eu II .................. & $<2.40$ & $<-0.11$ & 1 & $\ldots$ & $\ldots$ & $\ldots$ & $\ldots$ & $\ldots$ & $\ldots$ & $\ldots$ & $\ldots$ & $\ldots$ & $<0.93$ & $<-1.47$ & 2 & 0.23 \\
\hline $\mathrm{Pb}$ I ........................... & $<3.17$ & $<2.11$ & 1 & $\ldots$ & 2.18 & 1.75 & 1 & $\ldots$ & $\ldots$ & $\ldots$ & $\ldots$ & $\ldots$ & 2.61 & 1.65 & 1 & $\ldots$ \\
\hline
\end{tabular}

a The meteoritic abundance is used for $\mathrm{Li}$, rather than the solar one.

b This is $[\mathrm{Fe} / \mathrm{H}]$.

TABLE 11

Abundances for the Last Three EMP C Stars from the HES

\begin{tabular}{|c|c|c|c|c|c|c|c|c|c|c|c|c|}
\hline \multirow[b]{2}{*}{ SPECIES } & \multicolumn{4}{|c|}{ HE $2158-0348$} & \multicolumn{4}{|c|}{ HE $2232-0603$} & \multicolumn{4}{|c|}{ HE 2356-0410 } \\
\hline & $\begin{array}{c}{[\mathrm{X} / \mathrm{Fe}]} \\
(\mathrm{dex})\end{array}$ & $\begin{array}{c}\log \epsilon(\mathrm{X}) \\
\quad(\mathrm{dex})\end{array}$ & $\begin{array}{c}\text { No. } \\
\text { Lines }\end{array}$ & $\begin{array}{c}\sigma \\
(\operatorname{dex})\end{array}$ & $\begin{array}{c}{[\mathrm{X} / \mathrm{Fe}]} \\
(\mathrm{dex})\end{array}$ & $\begin{array}{l}\log \epsilon(X) \\
\quad(\operatorname{dex})\end{array}$ & $\begin{array}{c}\text { No. } \\
\text { Lines }\end{array}$ & $\begin{array}{c}\sigma \\
(\operatorname{dex})\end{array}$ & $\begin{array}{c}{[\mathrm{X} / \mathrm{Fe}]} \\
(\mathrm{dex})\end{array}$ & $\begin{array}{c}\log \epsilon(X) \\
(\operatorname{dex})\end{array}$ & $\begin{array}{c}\text { No. } \\
\text { Lines }\end{array}$ & $\begin{array}{c}\sigma \\
(\operatorname{dex})\end{array}$ \\
\hline $\mathrm{C}(\mathrm{CH}) \ldots \ldots \ldots \ldots \ldots \ldots \ldots \ldots \ldots \ldots \ldots \ldots \ldots \ldots$ & 1.87 & 7.76 & 1 & $\ldots$ & 1.22 & 7.96 & 1 & $\ldots$ & 2.14 & 7.66 & 1 & $\ldots$ \\
\hline $\mathrm{N}(\mathrm{CN})$ & 1.52 & 6.75 & 1 & $\ldots$ & 0.47 & 6.55 & 1 & $\ldots$ & 1.89 & 6.75 & 1 & $\ldots$ \\
\hline 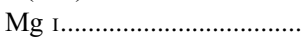 & 0.68 & 5.52 & 3 & 0.18 & 0.85 & 6.54 & 5 & 0.32 & 0.36 & 4.83 & 3 & 0.50 \\
\hline 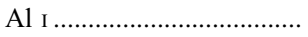 & 0.47 & 4.24 & 1 & $\ldots$ & 0.26 & 4.88 & 1 & $\ldots$ & 0.25 & 3.65 & 1 & $\ldots$ \\
\hline 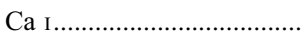 & 0.82 & 4.48 & 2 & 0.05 & 0.35 & 4.86 & 2 & 0.15 & 0.71 & 4.01 & 2 & 0.13 \\
\hline 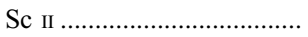 & $\ldots$ & $\ldots$ & $\ldots$ & $\ldots$ & 0.05 & 1.31 & 1 & $\ldots$ & 0.48 & 0.51 & 1 & $\ldots$ \\
\hline 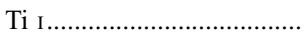 & 0.43 & 2.72 & 6 & 0.24 & 0.35 & 3.50 & 13 & 0.21 & 0.25 & 2.17 & 6 & 0.13 \\
\hline 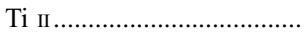 & 0.65 & 2.94 & 8 & 0.28 & 0.11 & 3.25 & 11 & 0.17 & 0.33 & 2.25 & 8 & 0.23 \\
\hline 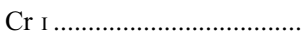 & -0.66 & 2.31 & 1 & $\ldots$ & -0.06 & 3.76 & 3 & 0.25 & -0.36 & 2.24 & 1 & $\ldots$ \\
\hline 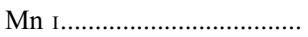 & -0.19 & 2.50 & 2 & 0.01 & -0.41 & 3.14 & 3 & 0.34 & -0.10 & 2.22 & 4 & 0.28 \\
\hline Fe I & $-2.70^{\mathrm{a}}$ & 4.75 & 28 & 0.26 & $-1.85^{\mathrm{a}}$ & 5.60 & 38 & 0.23 & $-3.07^{\mathrm{a}}$ & 4.38 & 28 & 0.21 \\
\hline 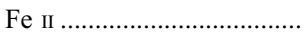 & -0.03 & 4.72 & 8 & 0.12 & -0.28 & 5.32 & 7 & 0.13 & -0.06 & 4.32 & 8 & 0.15 \\
\hline 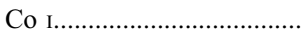 & -0.06 & 2.16 & 1 & $\ldots$ & 0.02 & 3.09 & 3 & 0.06 & 0.18 & 2.03 & 1 & $\ldots$ \\
\hline 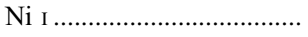 & $\ldots$ & $\ldots$ & $\ldots$ & $\ldots$ & -0.65 & 3.76 & 1 & $\ldots$ & $\ldots$ & $\ldots$ & $\ldots$ & $\ldots$ \\
\hline 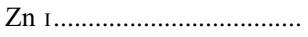 & $\ldots$ & $\ldots$ & $\ldots$ & $\ldots$ & $\ldots$ & $\ldots$ & $\ldots$ & $\ldots$ & 0.51 & 2.04 & 1 & $\ldots$ \\
\hline 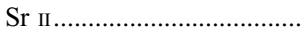 & 0.52 & 0.72 & 1 & $\ldots$ & 0.55 & 1.61 & 1 & $\ldots$ & -0.98 & -1.15 & 1 & $\ldots$ \\
\hline 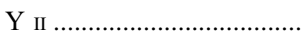 & 0.87 & 0.41 & 3 & 0.19 & 0.60 & 0.99 & 3 & 0.24 & $<-0.01$ & $<-0.84$ & 2 & 0.25 \\
\hline 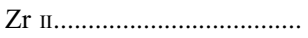 & 1.74 & 1.64 & 2 & 0.32 & $\ldots$ & $\ldots$ & $\ldots$ & $\ldots$ & $\ldots$ & $\ldots$ & $\ldots$ & $\ldots$ \\
\hline 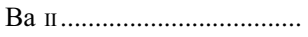 & 1.59 & 1.02 & 2 & 0.15 & 1.41 & 1.69 & 3 & 0.19 & -0.78 & -1.72 & 2 & 0.25 \\
\hline 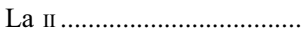 & 1.55 & 0.00 & 4 & 0.21 & 1.23 & 0.53 & 3 & 0.27 & $<0.60$ & $<-1.33$ & 1 & $\ldots$ \\
\hline 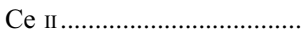 & 1.89 & 0.75 & 5 & 0.21 & 1.45 & 1.15 & 1 & $\ldots$ & $<1.04$ & $<-0.48$ & 1 & $\ldots$ \\
\hline 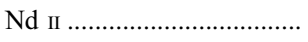 & 1.51 & 0.31 & 2 & 0.23 & $\ldots$ & $\ldots$ & $\cdots$ & $\cdots$ & $<1.20$ & $<-0.36$ & 4 & 0.26 \\
\hline 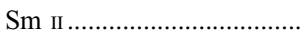 & $<2.40$ & $<0.71$ & 1 & $\ldots$ & $\ldots$ & $\ldots$ & $\ldots$ & $\ldots$ & $\ldots$ & $\ldots$ & $\ldots$ & $\ldots$ \\
\hline 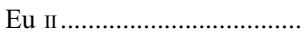 & 0.80 & -1.38 & 2 & 0.15 & $\ldots$ & $\ldots$ & $\ldots$ & $\ldots$ & $<0.97$ & $<-1.59$ & 2 & 0.35 \\
\hline 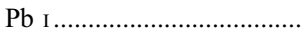 & 2.60 & 1.85 & 1 & $\ldots$ & 1.55 & 1.65 & 1 & $\ldots$ & $\ldots$ & $\ldots$ & $\ldots$ & $\ldots$ \\
\hline
\end{tabular}

${ }^{\mathrm{a}}$ This is $[\mathrm{Fe} / \mathrm{H}]$. 
TABLE 12

${ }^{12} \mathrm{C} /{ }^{13} \mathrm{C}$ Ratios For EMP C STARs From the HES

\begin{tabular}{|c|c|c|}
\hline ID & ${ }^{12} \mathrm{C} /{ }^{13} \mathrm{C}\left(\mathrm{C}_{2}\right)^{\mathrm{a}}$ & ${ }^{12} \mathrm{C} /{ }^{13} \mathrm{C}(\mathrm{CH})$ \\
\hline 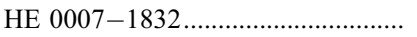 & $>2.0$ & $\ldots$ \\
\hline 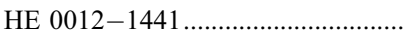 & $>3.0$ & $\ldots$ \\
\hline 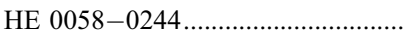 & 3.5 & $8-10$ \\
\hline 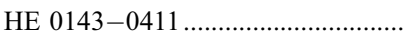 & $>4.0$ & $\ldots$ \\
\hline 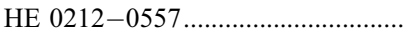 & 4.0 & $3-4$ \\
\hline 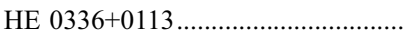 & 2.5 & 7.5 \\
\hline 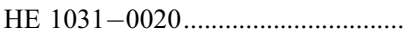 & 5.0 & $\ldots$ \\
\hline 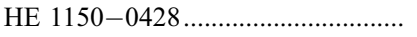 & 4.0 & $\ldots$ \\
\hline 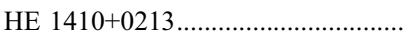 & 2.0 & 2.5 \\
\hline 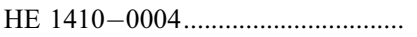 & $>3.0$ & $\ldots$ \\
\hline 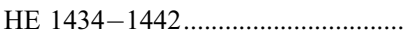 & 5.0 & $\ldots$ \\
\hline 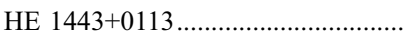 & 5.0 & $\ldots$ \\
\hline 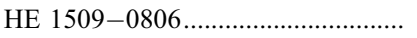 & 4.0 & $\ldots$ \\
\hline 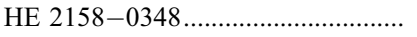 & 6.0 & $3-5$ \\
\hline 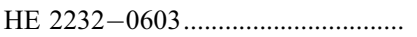 & $>6.0$ & $\geq 30$ \\
\hline 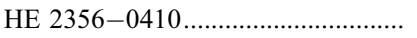 & 4.0 & $3-5$ \\
\hline
\end{tabular}

a The uncertainty in the deduced ${ }^{12} \mathrm{C} /{ }^{13} \mathrm{C}$ ratios is $30 \%$ of the isotopic ratio.
4213.1, 4219.2, and 4221.8 $\AA$ were used. We have verified for three stars whose Subaru HDS spectra were supplied by W. Aoki that our line list combined with our standard analysis procedures gives ${ }^{12} \mathrm{C} /{ }^{13} \mathrm{C}$ ratios derived from $\mathrm{CH}$ features differing from those derived by Aoki et al. $(2001,2002$ a) by $15 \%$ or less.

Spectrum synthesis for the $\mathrm{C}_{2}$ bands was carried out based on the $\mathrm{C}_{2}$ line list of Querci et al. $(1971,1974)$, as updated and supported on the Web site of U. Jørgensen. ${ }^{10}$ The dissociation potential for $\mathrm{C}_{2}$ was taken as $6.30 \mathrm{eV}$ (Urdahl et al. 1991). An isotopic line shift depends on the ratio of the reduced mass of the diatomic molecule $\mathrm{AB}, m_{\mathrm{A}} m_{\mathrm{B}} /\left(m_{\mathrm{A}}+m_{\mathrm{B}}\right)$, for its two isotopic variants. This ratio is 1.04 for $\mathrm{C}_{2}$ and only 1.007 for $\mathrm{CH}$ when considering ${ }^{12} \mathrm{C}$ versus ${ }^{13} \mathrm{C}$. Thus, as has been known for a long time, isotopic effects are considerably easier to detect in certain $\mathrm{C}_{2}$ bands than in those of $\mathrm{CH}$. For the $\mathrm{G}$ band of $\mathrm{CH}$, one must study detailed profiles of individual lines within the band that are often blends of multiple components of ${ }^{12} \mathrm{CH}$ or ${ }^{13} \mathrm{CH}$. The situation for $\mathrm{C}_{2}$ is very different. The strongest $\mathrm{C}_{2}$ band within our

\footnotetext{
${ }^{10}$ See http://www.astro.ku.dk/ uffegj/scan.
}

TABLE 13

Abundance Changes for Small Changes in Stellar Parameters

\begin{tabular}{|c|c|c|c|c|c|c|}
\hline \multirow[b]{2}{*}{ SPeCIES } & \multicolumn{6}{|c|}{$\Delta[\mathrm{X} / \mathrm{Fe}](\mathrm{dex})$} \\
\hline & $\left(T_{\text {eff }}-150 \mathrm{~K}\right)$ & $(\log g-0.4$ dex $)$ & Model $[\mathrm{Fe} / \mathrm{H}]-0.5$ & $\left(v_{t}-0.2 \mathrm{~km} \mathrm{~s}^{-1}\right)$ & $W_{\lambda}$ Unc. & $1 \mathrm{Star}(1 \sigma)^{\mathrm{a}}$ \\
\hline $\mathrm{C}(\mathrm{CH})$ & -0.18 & 0.14 & -0.04 & -0.02 & $\ldots$ & 0.23 \\
\hline $\mathrm{N}(\mathrm{CN})^{\mathrm{b}}$ & 0.04 & 0.09 & 0.00 & 0.00 & $\ldots$ & $0.26^{\mathrm{c}}$ \\
\hline 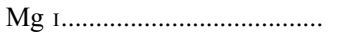 & 0.00 & 0.11 & 0.01 & 0.03 & 0.05 & 0.13 \\
\hline $\mathrm{Al}$ I & -0.02 & 0.10 & 0.02 & 0.10 & 0.08 & 0.16 \\
\hline 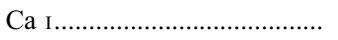 & 0.04 & 0.03 & 0.01 & 0.06 & 0.06 & 0.10 \\
\hline Ті г ........... & -0.01 & -0.01 & -0.01 & 0.02 & 0.03 & 0.04 \\
\hline Тi II & 0.08 & -0.14 & -0.01 & 0.11 & 0.03 & 0.20 \\
\hline Ti II ${ }^{\mathrm{d}}$ & -0.06 & 0.03 & 0.01 & 0.11 & 0.03 & 0.13 \\
\hline Cr I & 0.00 & -0.01 & -0.01 & 0.02 & 0.08 & 0.08 \\
\hline $\mathrm{Mn}_{\mathrm{I}}^{\mathrm{e}} \ldots \ldots \ldots \ldots$ & -0.06 & 0.05 & 0.02 & 0.16 & 0.06 & 0.19 \\
\hline $\mathrm{Fe}_{\mathrm{I}}^{\mathrm{f}} \ldots \ldots$ & $-0.16^{\mathrm{f}}$ & $0.03^{\mathrm{f}}$ & $0.13^{\mathrm{f}}$ & $0.08^{\mathrm{f}}$ & $0.02^{\mathrm{f}}$ & $0.22^{\mathrm{f}}$ \\
\hline Fe II & 0.14 & -0.17 & -0.02 & 0.03 & 0.03 & 0.23 \\
\hline Co $\mathrm{I}^{\mathrm{e}}$ & -0.02 & -0.01 & -0.01 & 0.03 & 0.08 & 0.09 \\
\hline 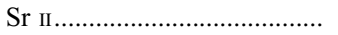 & 0.00 & -0.02 & 0.00 & 0.08 & 0.08 & 0.11 \\
\hline $\mathrm{Sr}_{\mathrm{II}}^{\mathrm{d}}$ & -0.14 & 0.15 & 0.02 & 0.08 & 0.08 & 0.24 \\
\hline 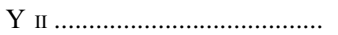 & 0.07 & -0.15 & -0.02 & 0.06 & 0.05 & 0.19 \\
\hline $\mathrm{Y} \mathrm{II}^{\mathrm{d}}$ & -0.07 & 0.02 & 0.00 & 0.06 & 0.05 & 0.11 \\
\hline 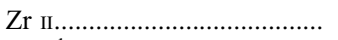 & 0.08 & -0.15 & -0.02 & 0.11 & 0.06 & 0.21 \\
\hline $\mathrm{Zr}$ II $^{\mathrm{d}}$ & -0.07 & 0.02 & 0.00 & 0.11 & 0.06 & 0.14 \\
\hline $\mathrm{Ba}$ II $^{\mathrm{e}}$ & -0.04 & 0.03 & 0.00 & 0.04 & 0.06 & 0.09 \\
\hline $\mathrm{Ba}$ II $^{\mathrm{d}, \mathrm{e}}$ & -0.19 & 0.20 & 0.02 & 0.04 & 0.06 & 0.28 \\
\hline $\mathrm{La}_{\mathrm{II}}^{\mathrm{e}}$ & 0.06 & -0.14 & -0.02 & 0.09 & 0.04 & 0.19 \\
\hline La II $\mathrm{d}, \mathrm{e}$ & -0.08 & 0.02 & 0.00 & 0.09 & 0.04 & 0.13 \\
\hline $\mathrm{Ce}$ II & 0.06 & -0.15 & -0.02 & 0.05 & 0.04 & 0.18 \\
\hline $\mathrm{Ce}_{\mathrm{II}}^{\mathrm{d}}$ & -0.08 & 0.02 & 0.00 & 0.05 & 0.04 & 0.11 \\
\hline Nd II & 0.04 & -0.14 & -0.03 & 0.04 & 0.06 & 0.17 \\
\hline $\mathrm{Nd}_{\mathrm{II}}^{\mathrm{d}} \ldots \ldots$ & -0.10 & 0.02 & -0.01 & 0.04 & 0.06 & 0.13 \\
\hline 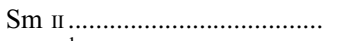 & 0.06 & -0.15 & -0.02 & 0.06 & 0.08 & 0.19 \\
\hline Sm II ${ }^{\mathrm{d}} \ldots \ldots$ & -0.08 & 0.02 & 0.00 & 0.06 & 0.08 & 0.13 \\
\hline $\mathrm{Eu}$ II $^{\mathrm{e}}$ & 0.05 & -0.15 & -0.02 & 0.03 & 0.06 & 0.18 \\
\hline $\mathrm{Eu}_{\mathrm{II}}^{\mathrm{d}, \mathrm{e}}$ & -0.09 & 0.02 & 0.00 & 0.03 & 0.06 & 0.11 \\
\hline $\mathrm{Pb}^{\mathrm{g}}$ & -0.05 & 0.12 & 0.02 & 0.14 & 0.08 & 0.21 \\
\hline
\end{tabular}

NotE.-Computed from the line list and the stellar parameters of HE 2158-0348 with respect to $\mathrm{Fe}$ I for all species.

${ }^{a}$ The $1 \sigma$ uncertainty in $[\mathrm{X} / \mathrm{Fe}]$ for a single (typical) star, including the five sources of uncertainties.

${ }^{b}$ Assumes $\log \epsilon(\mathrm{C})$ varies as for $\mathrm{CH}$.

c This includes the uncertainty in $\epsilon(\mathrm{C})$, as $\epsilon(\mathrm{N})$ is derived from lines of $\mathrm{CN}$.

${ }^{\mathrm{d}}$ Changes computed with respect to Fe II.

e Treated as individual absorption lines without HFS corrections.

${ }^{\mathrm{f}}$ Change in $\log \epsilon(\mathrm{Fe})$.

g The $4057 \AA$ feature is treated as a single $\mathrm{Pb}$ I line. 


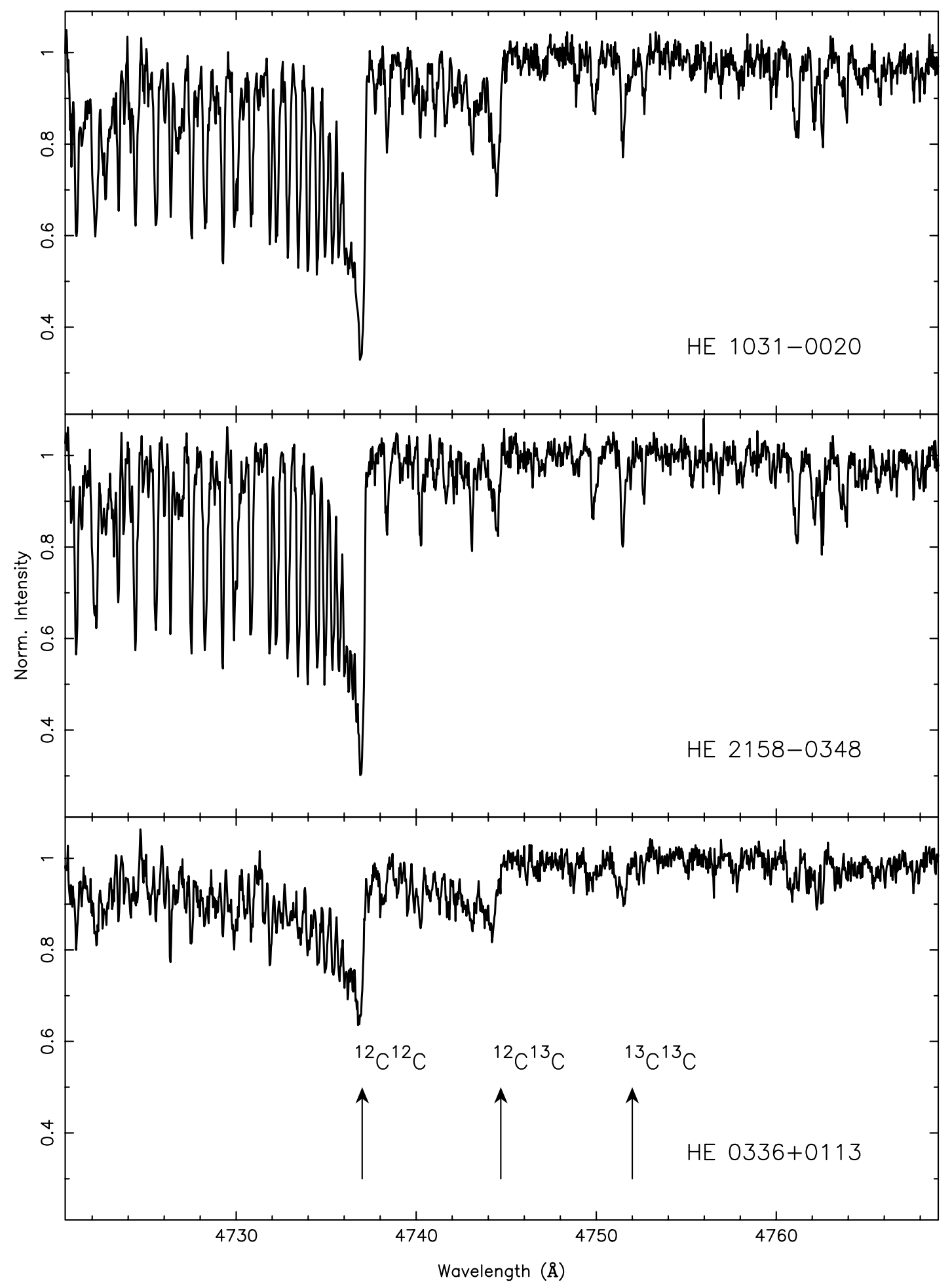

FIG. 2.- HIRES spectra of three $\mathrm{C}$ stars from our sample in the region of $4740 \AA$. The band heads of ${ }^{12} \mathrm{C}^{12} \mathrm{C},{ }^{12} \mathrm{C}^{13} \mathrm{C}$, and ${ }^{13} \mathrm{C}^{13} \mathrm{C}$ are indicated. The vertical range is the same for each panel. The derived ${ }^{12} \mathrm{C} /{ }^{13} \mathrm{C}$ ratio for these three stars ranges from 3.5 to 6 , identical to within the observational errors of $\pm 30 \%$.

spectral range is the $(0,0)$ Swan band at $5160 \AA$, which has a very small isotopic shift. However, the $(1,0)$ band head for ${ }^{12} \mathrm{C}^{13} \mathrm{C}$ at $4744 \AA$ is separated from that of ${ }^{12} \mathrm{C}^{12} \mathrm{C}$ at $4737 \AA$ by $\sim 7 \AA$, which is easily resolved even in moderate-resolution spectra. The ${ }^{13} \mathrm{C}^{13} \mathrm{C}$ band head is $\sim 8 \AA$ farther to the red at $4752 \AA$; it can be glimpsed in the $\mathrm{C}$ stars in our sample with the smallest ${ }^{12} \mathrm{C} /{ }^{13} \mathrm{C}$ ratios. Plates 26 and 29 of Keenan \& McNeil (1976) show examples of spectra of $\mathrm{C}$ stars with high and low ${ }^{12} \mathrm{C} /{ }^{13} \mathrm{C}$ ratios in this spectral region. Figure 2 illustrates the ease of separating the band heads ${ }^{12} \mathrm{C}^{12} \mathrm{C}$ and ${ }^{12} \mathrm{C}^{13} \mathrm{C}$ with the present much higher resolution data. Any uncertainty in the band electronic oscillator strength does not affect the determination of the ${ }^{12} \mathrm{C} /{ }^{13} \mathrm{C}$ ratio.

Because ${ }^{12} \mathrm{C}^{13} \mathrm{C}$ is a heteronuclear molecule and ${ }^{13} \mathrm{C}$ has a nonzero nuclear spin, it has a different number of states from ${ }^{12} \mathrm{C}^{12} \mathrm{C}$, affecting the partition function, as well as the number of transitions in a band. Since the spectrum synthesis program MOOG that we use does not distinguish between isotopic molecular species, it is necessary to reduce the $g f$-value of each 
${ }^{12} \mathrm{C}^{13} \mathrm{C}$ line by a factor of 2 to account for the partition function difference with ${ }^{12} \mathrm{C}^{12} \mathrm{C} ;{ }^{13} \mathrm{C}^{13} \mathrm{C}$ lines would require a factor of 4 reduction (e.g., see Amiot 1983). ${ }^{11}$ We note that the band oscillator strengths for the isotopic species may not be exactly equal due to wave function differences.

We performed a sanity check on our isotopic $\mathrm{C}_{2}$ band line lists by synthesizing the ${ }^{12} \mathrm{C}^{13} \mathrm{C}$ and ${ }^{12} \mathrm{C}^{12} \mathrm{C}(1,0)$ band heads. In this test we adopted a carbon abundance low enough that the band heads were unsaturated. If we set ${ }^{12} \mathrm{C} /{ }^{13} \mathrm{C}=1$, and synthesize the spectrum in the region of the band head of the $(1,0)$ Swan $C_{2}$ band, the ${ }^{12} \mathrm{C}^{13} \mathrm{C}$ and ${ }^{12} \mathrm{C}^{12} \mathrm{C}$ band heads should then be roughly equal in strength, because although the ${ }^{13} \mathrm{C}_{2}$ isotopic band head has twice as many lines, its partition function is a factor of 2 larger. The ratio of absorption at the appropriate resulting band heads in the synthesized spectrum is within $15 \%$ of unity, as expected.

\subsection{Ionization Equilibrium and Non-LTE}

Since we have not used the high-resolution spectra themselves to determine $T_{\text {eff }}$ or $\log g$, the ionization equilibrium is a stringent test of our analysis and procedures, including the determination of $T_{\text {eff }}$ and of $\log g$, as well as the assumption of LTE. For the 16 candidate EMP C stars from the HES we analyze here, the Fe ionization equilibrium is shown in Figure 3; we obtain a mean for $\log \epsilon(\mathrm{Fe}: \mathrm{Fe}$ II $)-\log \epsilon(\mathrm{Fe}: \mathrm{Fe}$ I $)$ of $-0.07 \mathrm{dex}$, with a $1 \sigma \mathrm{rms}$ scatter about the mean of 0.16 dex.

This is an extremely good ionization equilibrium for stars with such complex spectra, and it demonstrates the validity of our determination of stellar parameters from photometry and isochrones. The ionization equilibrium for Ti is almost as good, with a mean of $+0.08 \mathrm{dex}, \sigma=0.28 \mathrm{dex}$. The dispersion falls to $0.22 \mathrm{dex}$ (and the mean becomes $-0.03 \mathrm{dex}$ ) if one outlier with extremely weak Ti I lines is eliminated.

The Fe abundances derived from the neutral and ionized lines shift out of equilibrium by $\sim 0.25$ dex for a $250 \mathrm{~K}$ change in $T_{\text {eff }}$ in this temperature regime (see Table 13). Our adopted uncertainty in $T_{\text {eff }}$ is $\pm 150 \mathrm{~K}$, and the resulting uncertainty in $\log g$ is discussed in $\S 2.1$. Table 13 demonstrates that these two factors alone can give rise to the dispersion observed among the sample stars in the Fe ionization equilibrium.

Following Cohen et al. (2004), we implement a non-LTE correction to $\log \epsilon(\mathrm{Al})$ of +0.60 dex for the lines of the $\mathrm{Al}$ I doublet near $3950 \AA$ (Baumüller \& Gehren 1997). Only the $3961 \AA$ line can be used for most of these $C$ stars; the other line of this doublet is blended with molecular features. The $3905 \AA$ line of Si I, the only suitable line of this element in the wavelength range covered in most of our spectra, is also heavily blended with $\mathrm{CH}$ lines; we do not use it. Si abundances have been determined only for the small number of $\mathrm{C}$ stars with the redder wavelength coverage achieved with the new HIRES detector, with which unblended Si I lines near $5800 \AA$ A become available. We use a non-LTE correction for $\mathrm{Na}$ abundances from the $5889,5895 \AA$ doublet of $-0.20 \mathrm{dex}$ following Baumüller et al. (1998) and Takeda et al. (2003). Only one star (HE 1410-0004) has a spectrum that reaches any of the O I features, yielding an upper limit to the $6300 \AA$ forbidden line and a marginal detection of the strongest line in the triplet at $7772 \AA$ A. Kisselman (2001) pointed out the need for non-LTE corrections for the IR triplet line, and we use those calculated by Takeda (2003). We adopt a non-LTE correction for $\mathrm{O}$ for this star of -0.2 dex.

\footnotetext{
${ }^{11}$ Notes on the Web site of U. Jørgensen (http://www.astro.ku.dk/ uffegj/ scan) suggest that this argument may be too simplistic, and that a factor of 4 should be used instead to correct the $g f$-values for the ${ }^{12} \mathrm{C}^{13} \mathrm{C}$ lines. If true, our derived ${ }^{13} \mathrm{C}$ abundances will need to be reduced by a factor of 2 .
}

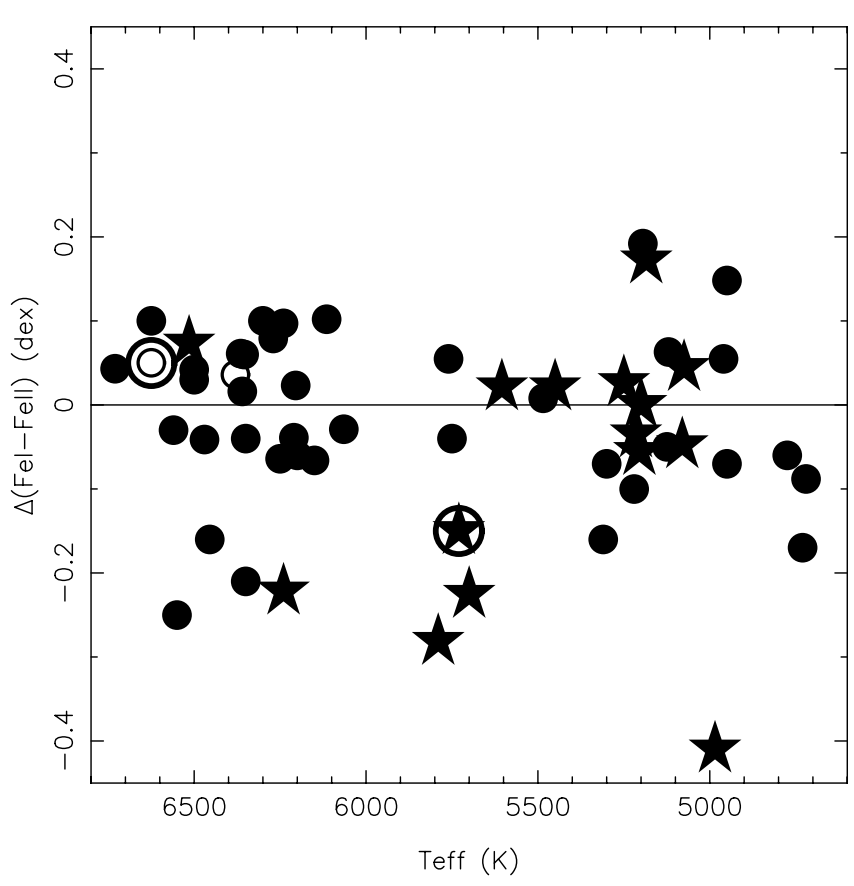

FIG. 3.-Fe ionization equilibrium for the 16 very metal-poor and EMP C stars in the present sample. The $\mathrm{C}$ stars are indicated as large stars, the $\mathrm{C}$-enhanced stars as open circles, and the $\mathrm{C}$-normal stars from our published and unpublished analyses as filled circles. The three known spectroscopic binaries in this sample are circled. [One of these is an apparently C-normal star (HE 0218-2736) that is too hot and too metal-poor to show any Fe II lines, and hence does not appear in this figure.]

\subsection{Comparison with Previous High-Dispersion Analyses}

Two of the $16 \mathrm{C}$ stars studied here are rediscoveries of stars found in the HK survey (Beers et al. 1985, 1992) and have been previously observed at high dispersion. HE 0058-0244 (CS 22183-015) was analyzed by Johnson \& Bolte (2002). They relied on stellar parameters determined from the spectra themselves; their adopted $T_{\text {eff }}(5200 \pm 100 \mathrm{~K})$ is $400 \mathrm{~K}$ lower than our value, and their $\log g$ is correspondingly 1 dex higher to preserve ionization equilibrium. The difference in their derived $[\mathrm{Fe} / \mathrm{H}]$, which is 0.35 dex lower than our value, is due entirely to the differences in the adopted stellar parameters. We attempt to compare $[\mathrm{X} / \mathrm{Fe}]$, modifying their values to our adopted $T_{\mathrm{eff}}$ and $\log g$ using the sensitivity table (Table 13). With these corrections, which in some cases are large, we find pretty good agreement (within $0.25 \mathrm{dex}$ ), except for [Y/Fe] and [ $\mathrm{La} / \mathrm{Fe}]$, for which our abundances are 0.4 dex lower than theirs.

Norris et al. (1997), Bonifacio et al. (1998), Preston \& Sneden (2001), and recently Aoki et al. (2002b) observed HE 23560410 (CS 22957-027). The first two groups used $B-V$ to establish $T_{\text {eff }}$; they both used a value $350 \mathrm{~K}$ cooler than that we adopted here. Preston \& Sneden (2001) used a hybrid method with $B-V$ corrected for molecular absorption to determine $T_{\text {eff }}$, while Aoki et al. (2002b) used $B-V$ and $V-K$ for this purpose, ending up with a value for $T_{\text {eff }}$ only $100 \mathrm{~K}$ lower than ours. These differences in adopted stellar parameters directly produce the differences in derived metallicity: the first two analyses yield [Fe/H] values 0.3 dex lower than adopted here, while that of the last is only 0.05 dex lower. Although there is overall good agreement for the $\mathrm{C}$ abundances, the derived [N/Fe] ranges over 0.9 dex among the five analyses.

We compare our derived abundance ratios with those of Aoki et al. (2002b), as the stellar parameters adopted in these two 
analyses are similar. Their ${ }^{12} \mathrm{C} /{ }^{13} \mathrm{C}$ ratio is $8 \pm 2$, in reasonable agreement with our value of $4.0 \pm 1.3$ (see Table 12). Our [C/Fe] is 0.2 dex lower than theirs, while our derived [N/Fe] is a similar amount larger, as it must be to compensate in order to fit the $\mathrm{CN}$ band strength. The abundance ratios for all the species in common agree fairly well, with $[\mathrm{Al} / \mathrm{Fe}],[\mathrm{Ca} / \mathrm{Fe}],[\mathrm{Sr} / \mathrm{Fe}]$, and $[\mathrm{Ba} / \mathrm{Fe}]$ showing the largest differences: $-0.42,{ }^{12}+0.58,-0.42$, and $+0.45 \mathrm{dex}$, respectively, for the values of $[\mathrm{X} / \mathrm{Fe}]$ derived here minus those of Aoki et al. (2002b). Large differences also occur in abundance ratios for many species in comparing our results with the other earlier analyses.

To isolate the cause of the large differences between the various analyses of these $\mathrm{C}$ stars, we have compared our measured $W_{\lambda}$ values with those published, when available. For HE 00580244 , the measured $W_{\lambda}$ values for the 12 weak lines in common (mean $W_{\lambda}$ of $27.7 \mathrm{~m} \AA$ ) with those tabulated by Johnson \& Bolte (2002) for $n$-capture elements agree with ours, with a mean difference of $1.1 \mathrm{~m} \AA$ and a $\sigma$ of $3.2 \mathrm{~m} \AA$. The only strong line in common is the $4554 \AA$ line of Ba II with a measured $W_{\lambda}$ of 177.5 and $166.2 \mathrm{~m} \AA$ in the two studies. For HE 2356-0410 we have 18 lines in common with those tabulated by Norris et al. (1997). The $W_{\lambda}$ values again agree well, with a mean difference of $0.3 \mathrm{~m} \AA$ and a $\sigma$ of $12.2 \mathrm{~m} \AA$. (The set of lines in common in this case are in general stronger lines, with a mean $W_{\lambda}$ of $62 \mathrm{~m} \AA$.) The agreement with the $W_{\lambda}$ for this star tabulated by Bonifacio et al. (1998) is also very good, with $\sigma$ of $6.1 \mathrm{~m} \AA$.

Thus, the differences in deduced abundances between the analysis presented here and those previously published for these two $\mathrm{C}$ stars are not due to differences in measured $W_{\lambda}$. They must arise from the choices made for the stellar parameters and in the details of the abundance analyses. In spite of these discrepancies, the overall characteristics of the abundance distribution in these two $\mathrm{C}$ stars are inferred as identical by each of the analyses. All five groups, for example, agree that HE 2356-0410 has an extremely large enhancement of $\mathrm{C}$ and has a very low $[\mathrm{Ba} / \mathrm{Fe}]$. The deviations from "normal" EMP stars are in general and for this particular star very large, larger than the errors made by any of the independent analyses.

We previously published an analysis of the dwarf $\mathrm{C}$ star $\mathrm{HE}$ 0143-0441 in Cohen et al. (2004). The analysis presented here supersedes that one; the adopted $T_{\text {eff }}$ is $130 \mathrm{~K}$ cooler due to acquisition of better optical photometry in the interim, and the $W_{\lambda}$ have also been rechecked for molecular blends since our earlier effort. The resulting $[\mathrm{Fe} / \mathrm{H}]$ is 0.14 dex smaller than that of our previous work. The abundance ratios $[\mathrm{X} / \mathrm{Fe}]$ derived from our two analyses are in good agreement, except for N. It appears there was a typographical error in the entry for $\log \epsilon(\mathrm{N})$ in Table 5 of Cohen et al. (2004), which is corrected in Table 8 here.

S. Lucatello et al. (2006, in preparation) will present a detailed abundance analysis for five of the $\mathrm{C}$ stars analyzed here. That analysis should be definitive, with extensive use of spectral syntheses and maximum care in all aspects. The Si abundance should be recoverable with such syntheses, and a careful synthesis of the region of the $3961 \AA$ line of $\mathrm{Al}$ I would improve the $\mathrm{Al}$ abundances presented here.

\section{COMMENTS ON INDIVIDUAL ELEMENTS}

\subsection{Iron}

We confirm that our $[\mathrm{Fe} / \mathrm{H}]$ determinations are largely free of molecular contamination by looking at the derived $\mathrm{Fe}$ abundance

\footnotetext{
12 The difference in the adopted non-LTE correction for the lines of the $3950 \AA$ doublet of $\mathrm{Al}$ I has been removed.
}

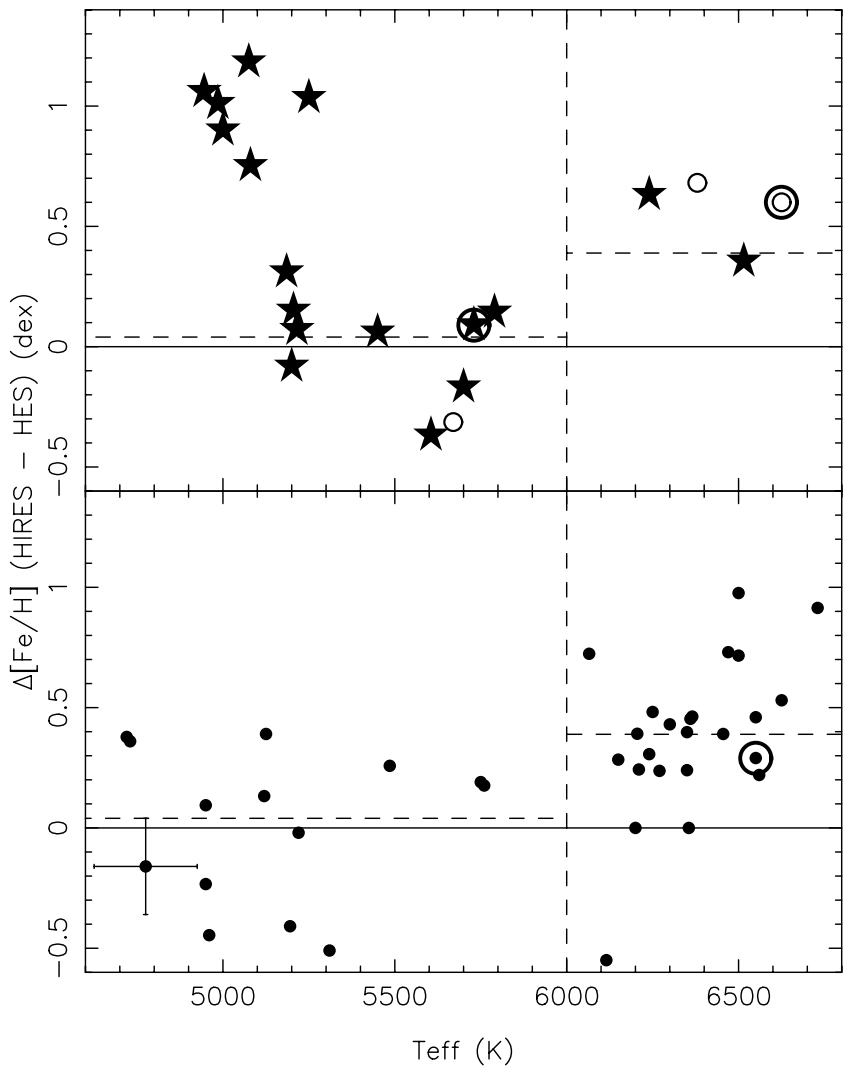

FIG. 4.-Difference between $[\mathrm{Fe} / \mathrm{H}](\mathrm{HES})$ and $[\mathrm{Fe} / \mathrm{H}](\mathrm{HIRES})$ as a function of $T_{\text {eff }}$ for the $\mathrm{C}$ stars (top) and for the C-normal stars (bottom) for those EMP candidates from the HES with analyses based on Keck HIRES spectra. The symbols are as in Fig. 3. The vertical dashed line separates the giants from the dwarfs, while the horizontal dashed lines represent the mean $\Delta$ for the C-normal giants and for the $\mathrm{C}$-normal dwarfs. A typical error is indicated for a single star.

in regions where molecular bands are absent as compared to those where they are (weakly) present. Regions where the molecular bands are strong in the spectrum of a sample star were ignored. Every star in our sample was checked to make sure that the Fe I abundance deduced from lines redward of $5160 \AA$ to the end of our spectral coverage, a region within which there are no molecular features, was the same as that for lines to the blue. For only two stars did a possible systematic difference appear, and it was only 0.1 dex, with the redder lines giving slightly lower Fe abundances. This supports the validity of our $\mathrm{Fe}$ abundances.

The $[\mathrm{Fe} / \mathrm{H}]$ values derived here are in some cases considerably higher than those predicted by the algorithm used on the moderate-resolution HES follow-up spectra. Figure 4 shows $\Delta[\mathrm{Fe} / \mathrm{H}]$, the difference between $[\mathrm{Fe} / \mathrm{H}]$ as determined from a detailed abundance analysis of high-dispersion spectra and that from the application of the Beers et al. (1999) algorithm to the moderate-resolution spectra. Initially, both for the HES and for the HK survey, the $B-V$ color was used to indicate $T_{\text {eff }}$. Such a procedure is very convenient for the HES, for example, as rough colors can be measured directly from the objective prism spectra. This procedure, however, is a disaster for $\mathrm{C}$ stars, as the $B$ bandpass is much more affected by molecular absorption from $\mathrm{CH}$ and $\mathrm{CN}$ than is the $V$ bandpass. Spuriously red $B-V$ colors lead to spuriously low deduced $T_{\text {eff }}$ values, which in turn lead to spuriously low deduced $\mathrm{Fe}$ abundances. In practice this affects all $\mathrm{C}$ stars cooler than $6000 \mathrm{~K}$, and almost certainly some even hotter than that. The literature is full of references to C-star abundance analyses in which the resulting high-resolution $[\mathrm{Fe} / \mathrm{H}]$ 
grossly (by $\sim 1$ dex) exceeds $[\mathrm{Fe} / \mathrm{H}](\mathrm{HK})$; see, for example, Norris et al. (1997) or Hill et al. (2000), for which the relevant $[\mathrm{Fe} / \mathrm{H}](\mathrm{HK})$ are given in Barbuy et al. (1997). The origin of this problem was realized several years ago by several groups; see, for example, Preston \& Sneden (2001). Both the HES and the HK survey then switched to using the strength of absorption at $\mathrm{H} \delta$ as a $T_{\text {eff }}$ indicator.

However, there is still a problem for the cooler $\mathrm{C}$ stars, as is shown in Figure 4. The five C stars HE 0212-0557, HE 10310020, HE 1434-1442, HE 1443+0113, and HE 1509-0806 show $\Delta[\mathrm{Fe} / \mathrm{H}] \sim 1$ dex. Something is still wrong, but now only the cooler giants, $T_{\text {eff }} \sim 5100 \mathrm{~K}$, and not all of them, are affected. As was shown by Cohen et al. (2005b), the problem is the molecular absorption in the specific bandpasses used, particularly in the red continuum bandpass for the $\mathrm{H} \delta$ index. For the most extreme $\mathrm{C}$ stars in our sample, the HP2 index measuring the H $\delta$ absorption defined and used by the HK survey becomes negative (i.e., implies that $\mathrm{H} \delta$ is in emission), which is not the case when one examines high-dispersion spectra. This again leads to spuriously low $T_{\text {eff }}$ estimates and hence to spuriously low deduced Fe abundances from the moderate-resolution follow-up HES or HK survey spectra. Both $\mathrm{CN}$ and $\mathrm{CH}$ contribute to the absorption there, with that of $\mathrm{CN}$ dominating at solar metallicity in the relevant $T_{\text {eff }}$ range. At the low $\mathrm{Fe}$ metallicities considered here, the relative contributions of $\mathrm{CN}$ and $\mathrm{CH}$ will depend primarily on $T_{\text {eff }}$, with the $\mathrm{C} / \mathrm{N}$ ratio and $\mathrm{Fe}$ abundance also playing a role. The five $\mathrm{C}$ stars with large $\Delta[\mathrm{Fe} / \mathrm{H}]$ (those discrepant in Fig. 4) are the five stars with the strongest absorption over the specific spectral region of interest (4144-4164 $\AA$ ).

In the regime of KP and HP2 corresponding to EMP giants, a change in HP2 of $0.5 \AA$ can produce a change in predicted $[\mathrm{Fe} / \mathrm{H}](\mathrm{HES})$ of $0.5 \mathrm{dex}$. The filter bandpass of HP2 is $12 \AA$ wide (Beers et al. 1999). Thus, a $0.5 \AA$ error in the measured HP2 index corresponds to a $4 \%$ error in the continuum level. Looking at the spectra of the coolest C stars in the 4000-4200 A region shown in Figure 2 of Cohen et al. (2005b) in the relevant region for the feature and sideband bandpasses of HP2, it is difficult to see how an underestimate of the continuum level of this size will not occur.

Thus, the algorithm adopted by the HES, and until recently the HK survey (Rossi et al. 2005), to deduce an Fe metallicity from low-dispersion spectra systematically underestimates $[\mathrm{Fe} / \mathrm{H}](\mathrm{HES})$ by a factor of $\sim 10$ for certain cool C stars $\left(T_{\text {eff }} \lesssim 5100 \mathrm{~K}\right)$. The important implications of this are the overestimate of the frequency of C stars among EMP stars, and the overestimate of the yield of EMP stars in the HES and, by implication, the HK survey. These issues are discussed briefly in Cohen et al. (2005b) and will be discussed at length in J. G. Cohen et al. (2006b, in preparation).

We demonstrate that the systematic $[\mathrm{Fe} / \mathrm{H}]$ underestimate for EMP C stars does not arise from the random uncertainty in the measurement of the HP2 indices. Comparison of HP2 indices measured from moderate-dispersion spectra for 57 stars, most of which are C-normal, with observations on different runs at the Palomar Observatory 200 inch $(5.1 \mathrm{~m})$ telescope (P200) or observed at both the P200 and Magellan telescopes shows a mean difference in measured HP2 indices of $0.18 \AA$, with an rms dispersion about the mean of $0.65 \AA$; details will be presented in J. G. Cohen et al. (2006b, in preparation). Also note that the moderateresolution spectra of the five $\mathrm{C}$ stars that show large $\Delta[\mathrm{Fe} / \mathrm{H}]$ (HES) are from four different runs with the Double Spectrograph on the Hale Telescope.

The slight overlap of high and low $\Delta[\mathrm{Fe} / \mathrm{H}](\mathrm{HES})$ values at the boundary in $T_{\text {eff }}$ where this effect becomes important $(\sim 5200 \mathrm{~K})$ can be explained as resulting from observational uncertainties; recall that our adopted uncertainty in $T_{\text {eff }}$ for these
$\mathrm{C}$ stars is $150 \mathrm{~K}$. Furthermore, this effect depends on the $\mathrm{C}$ abundance, the $\mathrm{C} / \mathrm{N}$ ratio, and to a smaller extent $[\mathrm{Fe} / \mathrm{H}]$, although the primary dependence is on $T_{\text {eff. }}$. Clearly, while using $\mathrm{H} \delta$ is better than using $B-V$ as a $T_{\text {eff }}$ indicator, it has its limitations, particularly for cool C-rich giants, as shown here. Using a $V-K$ color is better. $J-K$ is not useful for the faint stars found in the HES; the errors of the 2MASS database are too large compared to the sensitivity of $J-K$ to $T_{\text {eff }}$, which is, as discussed in $\S 2.1$, small. This statement may not hold for the HK survey, in which the stars are in the mean significantly brighter than the HES, and hence the 2MASS errors are much smaller.

\subsection{Carbon and Nitrogen}

Since a band of $\mathrm{CN}$ is used to derive the $\mathrm{N}$ abundance, the $\mathrm{N}$ abundance is linked to the choice of C. Systematic errors not included in Table 13 in the $\mathrm{C}$ and $\mathrm{N}$ abundances are possible in the case of unusually large oxygen abundance, because the $\mathrm{CN}$ and $\mathrm{CH}$ densities depend on the amount of free carbon left given $\mathrm{CO}$ formation.

HE $1150-0428$ has extremely strong $\mathrm{CN}$ bands; the band heads at 3885,3875 , and $3865 \AA$ are all present, and the first two of these reach a maximum absorption of $\sim 85 \%$ of the continuum. The continuum was very difficult to define in this region of the spectrum of this star. Combining that with saturation issues, the $\mathrm{N}$ abundance of this star is not well determined; appropriate errors might be $\log \epsilon(\mathrm{N})=7.15_{-0.3}^{+0.5}$ dex.

The determination of ${ }^{12} \mathrm{C} /{ }^{13} \mathrm{C}$ from the $\mathrm{C}_{2}$ and $\mathrm{CH}$ bands is described in $\S 3$ 3.2. Figure 5 displays the measured absorption of the stellar continuum at ${ }^{12} \mathrm{C}^{12} \mathrm{C}$ and ${ }^{12} \mathrm{C}^{13} \mathrm{C}$ band heads for the $\mathrm{C}$ stars in our sample; see also Figure 2. The deduced isotopic ratios for $\mathrm{C}$ derived from these two bands are shown as a function of $T_{\text {eff }}$ in Figure $6 .{ }^{12} \mathrm{C} /{ }^{13} \mathrm{C}$ is easy to determine from the $4740 \AA$ $\mathrm{C}_{2}$ band, and the many values given in Table 12 demonstrate that the ${ }^{12} \mathrm{C} /{ }^{13} \mathrm{C}$ ratio is low, with a typical value of 4 . The isotopic ratios for our sample of $\mathrm{C}$ stars as determined from $\mathrm{C}_{2}$ bands, ignoring the lower limits, are consistent to within $1.5 \sigma$ with a constant value of ${ }^{12} \mathrm{C} /{ }^{13} \mathrm{C}$ of $\sim 3.5$. Similar values have been found among luminous moderately metal-poor field giant stars with normal $C$ abundances and with luminosities near the tip of the RGB by Gratton et al. (2000). The hottest stars in the sample yield only lower limits to ${ }^{12} \mathrm{C} /{ }^{13} \mathrm{C}$ using either of the molecular features. More measurements of ${ }^{12} \mathrm{C} /{ }^{13} \mathrm{C}$ ratios at the extremes of the range of $T_{\text {eff }}$ would be required to search for any trend with $T_{\text {eff }}$.

\subsection{Barium}

In many cases, the $\mathrm{Ba}$ II lines are very strong, and the resulting derived $\mathrm{Ba}$ abundances must be regarded as quite uncertain. Their HFS corrections are sometimes large and vary considerably with $W_{\lambda}$. The HFS corrections calculated by McWilliam (1998) that we adopt are for an $r$-process isotopic distribution. We have rescaled them for the $s$-process $\mathrm{Ba}$ isotopic distribution; this in general reduces the deduced $\mathrm{Ba}$ abundance by $\sim 0.1$ dex.

\subsection{Lead}

There is only one usable $\mathrm{Pb}$ I line in the spectral region we cover. This line, at $4057.8 \AA$, is badly blended with CH features in these $\mathrm{C}$ stars. Our spectral synthesis for this feature uses the isotopic and HFS pattern for $\mathrm{Pb}$ described in $\S 3.1$, as well as features of ${ }^{12} \mathrm{CH},{ }^{13} \mathrm{CH}$, and various atomic species. A reasonable uncertainty for our $\mathrm{Pb}$ measurements based on spectral synthesis is \pm 0.3 dex. Nondetections, in cases in which there is no problem indicated by notes in Tables $8-11$, correspond to upper limits of $\log \epsilon(\mathrm{Pb})=+1.5$ dex. 


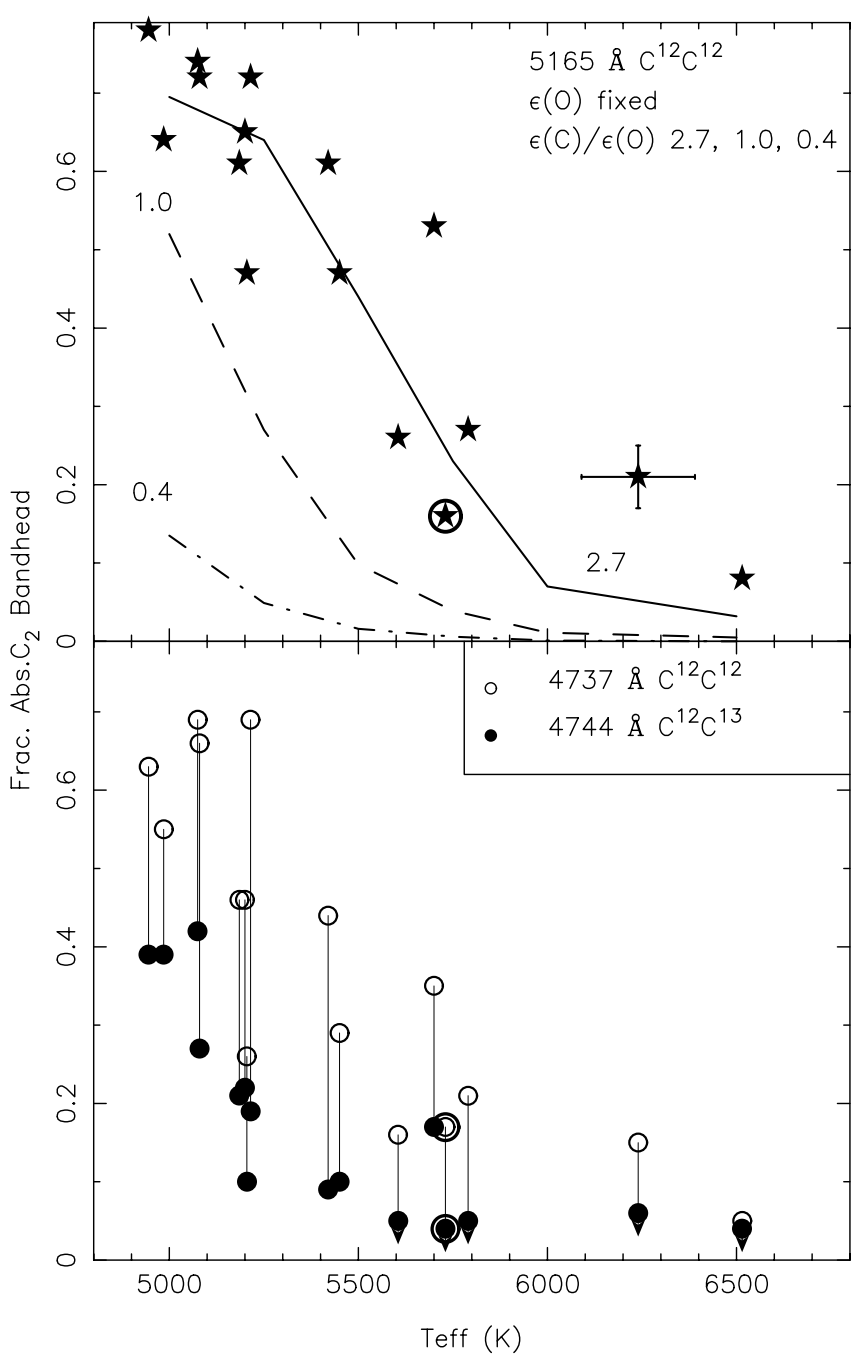

FIG. 5.-Fractional absorption at several $\mathrm{C}_{2}$ band heads measured from our Keck HIRES spectra for our sample of $\mathrm{C}$ stars from the HES as a function of $T_{\text {eff }}$. The symbols are as in Fig. 3. Top: Fractional absorption at $5165.0 \AA$ (the 0,0 band head of the Swan system). The solid curve indicates the predicted behavior for $\log \epsilon(\mathrm{C}), \log \epsilon(\mathrm{N})$, and $\log \epsilon(\mathrm{O})=7.56,6.55$, and $7.13 \mathrm{dex}(\mathrm{C} / \mathrm{O}=$ 2.7), the dashed curve is that for $\mathrm{C} / \mathrm{O}=1$, and the dot-dashed curve for $\mathrm{C} / \mathrm{O}=$ 1/2.7, keeping $\epsilon(\mathrm{O})$ fixed. Bottom: The $4737 \AA$ band head of ${ }^{12} \mathrm{C}^{12} \mathrm{C}$ as open circles, and the $4744 \AA$ band head of ${ }^{12} \mathrm{C}^{13} \mathrm{C}$ as filled circles. Vertical lines connect the two values for each C star. All C stars in our sample hotter than $5500 \mathrm{~K}$ only have upper limits for the latter.

\subsection{Use of Strong Lines}

It is desirable in carrying out a detailed abundance analysis to use only absorption lines with $W_{\lambda}$ less than $\sim 170 \mathrm{~m} \AA$ to keep the errors as small as possible. Stronger lines will be formed in the outermost layers of the stellar atmosphere, where the $T(\tau)$ relationship is more uncertain and where LTE is less likely to prevail. Hence, the $W_{\lambda}$ values predicted from a model atmosphere for such strong lines are more uncertain, as is the derived abundance of the species from which the line originates. However, the wavelength coverage of our spectra, almost all of which were taken prior to the HIRES detector upgrade, is restricted, and $\mathrm{CH}$, $\mathrm{CN}$, and $\mathrm{C}_{2}$ molecular bands in the spectra of these stars further cut down the useful wavelength range. Some elements have very few detectable lines of any state of ionization within the allowed region. In a few cases only strong lines are available, while in others one or two weak lines are sometimes present together with the strong ones, at least for a few of the $\mathrm{C}$ stars in our sample.

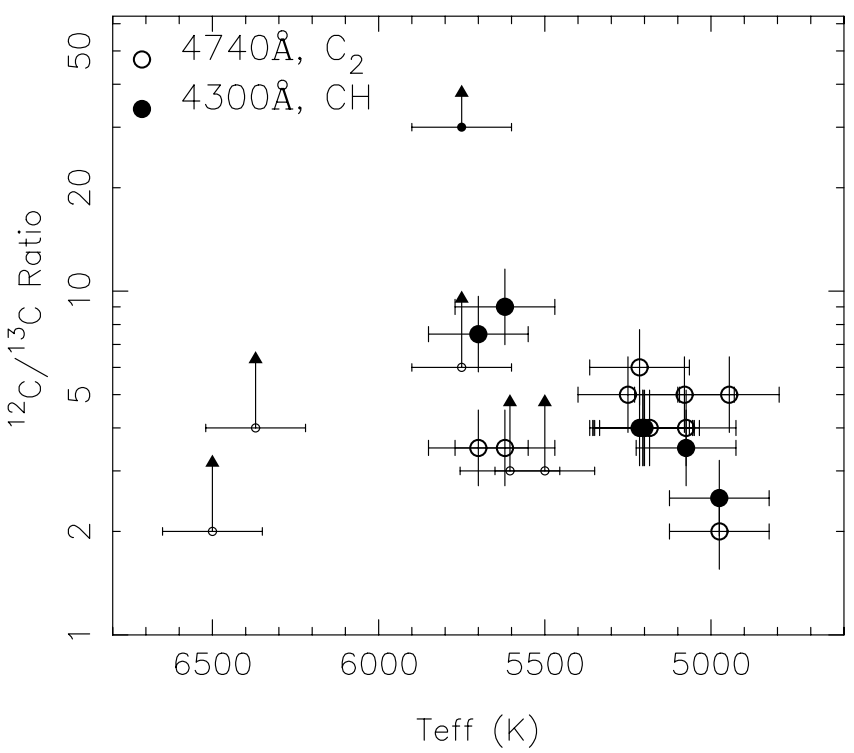

FIG. 6.-Plot of ${ }^{12} \mathrm{C} /{ }^{13} \mathrm{C}$ ratios measured from the $\mathrm{C}_{2}(1,0)$ Swan band and from the $G$ band of $\mathrm{CH}$ as a function of $T_{\mathrm{eff}}$ for the $\mathrm{C}$ stars in our sample. All C stars with $T_{\text {eff }}>5700 \mathrm{~K}$ have only lower limits to the ${ }^{12} \mathrm{C} /{ }^{13} \mathrm{C}$ ratio from the present spectra.

Examination of Tables 3-6 reveals the elements of concern. The $\mathrm{Na}$ I $\mathrm{D}$ lines are too strong for reliable abundance analysis in the spectrum of our coolest C star, HE $1443+0113$, and are the only lines detected of that species in the only available HIRES spectrum of that star, which has low $\mathrm{S} / \mathrm{N}$. Two lines of the $\mathrm{Mg}$ triplet at $5170 \AA$ are always detected (the third is blended and not used) and are sometimes stronger than $170 \mathrm{~m} \AA$, but often one or more of the weaker subordinate $\mathrm{Mg}$ I lines are seen as well. The Sr II line at $4077 \AA$ is the only one measured in many of the sample stars, as the $4215 \AA$ line is often swamped by CN. In the most $s$-process-enhanced cool C stars, this line exceeds the $W_{\lambda}$ cutoff suggested above; unfortunately, there are no other detectable $\mathrm{Sr}$ lines in the available wavelength region. The $\mathrm{Ba}$ II lines at 4554 and $4934 \AA$ are extremely strong, far beyond the limit in $W_{\lambda}$ suggested above, in several of the cooler Ba-rich C stars. But in many of these, the weaker $4130 \AA$ line is seen as well, and in the one star with a HIRES-R spectrum, the weaker 5854, 6141, and $6496 \AA \mathrm{Ba}$ II lines are picked up as well. Caution is necessary for these particular elements, but we believe that the magnitude of the potential errors is sufficiently small that the fundamental conclusions of our work are not affected.

\section{ABUNDANCE RATIOS}

\subsection{C/H Ratios}

We have two indicators in the present work for the carbon abundances, the strength of the bands of $\mathrm{CH}$ and of $\mathrm{C}_{2}$. Figure 7 (top) shows $\epsilon(\mathrm{C})$ inferred from the $G$ band of $\mathrm{CH}$ as a function of $[\mathrm{Fe} / \mathrm{H}](\mathrm{HIRES})$ for the full sample of $16 \mathrm{C}$ stars and three EMP $\mathrm{C}$-enhanced stars with $[\mathrm{C} / \mathrm{Fe}]>1.0$ dex from our work. Eleven additional very metal-poor $\mathrm{C}$ stars, mostly from the HK survey, with recent analyses from the literature, are indicated as small open circles in this figure, as well as in Figures 8 and 10. The details for the additional stars are given in Table 14. This produces a total sample of $27 \mathrm{Fe}$-poor $\mathrm{C}$ stars and three EMP C-enhanced dwarfs.

The dashed horizontal line in Figure 7 (top) indicates a constant $\mathrm{C} / \mathrm{H}$ ratio of $20 \%$ of the solar value independent of $[\mathrm{Fe} / \mathrm{H}]$. This constant $\epsilon(\mathrm{C})$, which we denote as $\epsilon_{0}(\mathrm{C})$, is a reasonable fit 


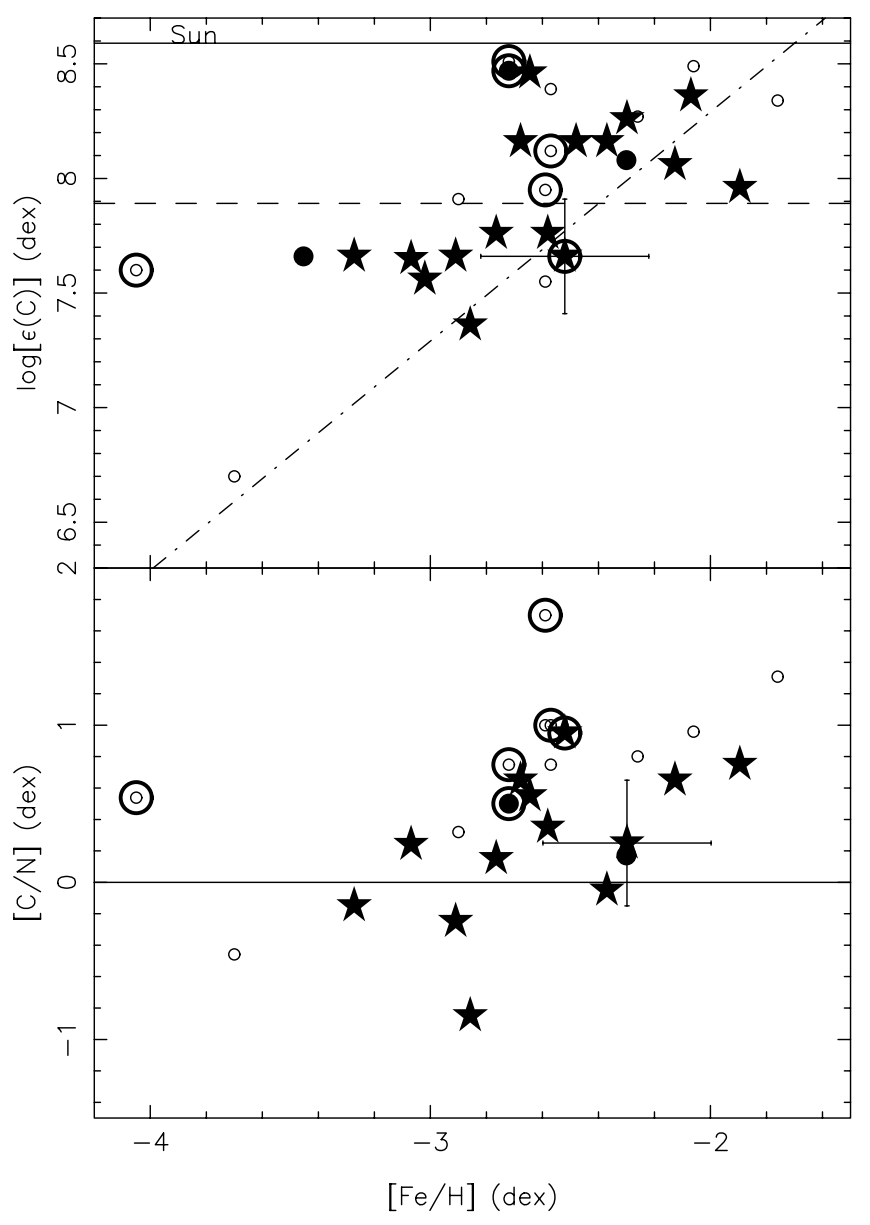

FIG. 7.-Top: Plot of $\log \epsilon(C)$ as a function of $[\mathrm{Fe} / \mathrm{H}]$ for $\mathrm{C}$ stars (large stars) and the C-enhanced stars ( filled circles) with detailed abundance analyses from the HES by our group. The augmented sample of very metal-poor $\mathrm{C}$ stars from the literature (see Table 14 for details) is shown as small open circles. Known spectroscopic binaries are circled. The dashed horizontal line indicates a fixed $\epsilon(\mathrm{C})$ of $20 \%$ that of the Sun. The sloping line indicates the locus of $[\mathrm{C} / \mathrm{Fe}]=$ +1.7 dex. Bottom: Same as the top panel, but for [C/N]. The horizontal line indicates the solar ratio. Typical error bars are indicated for a single star in each panel.

to all the available data, given the uncertainties. The inferred $\epsilon(\mathrm{C})$ reaches a maximum value of approximately one-third solar, consistent with $\epsilon_{0}(\mathrm{C})$. Figure 7 shows that EMP C stars, even though they are of very low $[\mathrm{Fe} / \mathrm{H}]$, can, by whatever processes are relevant, achieve $\mathrm{C}$ enrichment up to near the solar abundance, but not beyond it. This is also true of the two known ultrametal-poor stars (Christlieb et al. 2004; Frebel et al. 2005). Marsteller et al. (2005) also have reached similar conclusions using the HK survey sample.

The most metal-poor star shown in this figure is G77-61, with $[\mathrm{Fe} / \mathrm{H}]$ about $-4.0 \mathrm{dex}$. This star is an $\mathrm{M}$ dwarf in a binary system. Since the star is so cool compared to the $\mathrm{C}$ stars studied here, it has a much more complex spectrum with very strong molecular features. As part of a recent study by Plez \& Cohen (2005), a search was made for a detectable feature of $\mathrm{O}$ in the optical spectrum of this star. However, given the very strong molecular bands in this M dwarf, none could be found even in highprecision Keck HIRES spectra covering the full optical spectral regime from 0.4 to $1.0 \mu \mathrm{m}$. Thus, in the abundance analysis for G77-61 carried out by Plez \& Cohen (2005), it was assumed that $\mathrm{O}$ was enhanced by +0.3 dex (i.e., $[\mathrm{O} / \mathrm{Fe}]=+0.3$ dex). The resulting enhancement of $\mathrm{C}$ was found to be $[\mathrm{C} / \mathrm{Fe}]=+2.6$ dex. A recently obtained Keck high-resolution near-IR spectrum

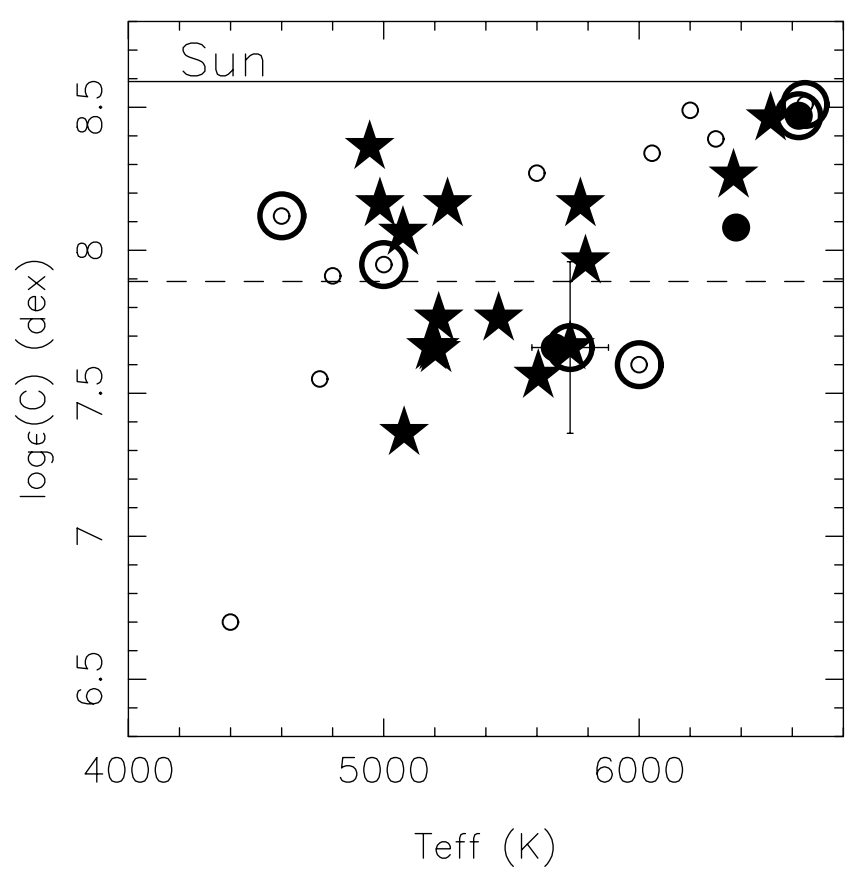

FIG. 8.-Plot of $\log \epsilon(C)$ as a function of $T_{\text {eff }}$ for C stars (large stars) and $\mathrm{C}$-enhanced stars (large filled circles) from our sample. The augmented sample of very metal-poor $\mathrm{C}$ stars with recent detailed abundance analyses from the literature (see Table 14 for details) is shown as small open circles. Known spectroscopic binaries are circled. The solid horizontal line represents the solar ratio, while the horizontal dashed line represents $20 \%$ of solar. G77-61 is plotted as a dwarf with $T_{\text {eff }}=6000 \mathrm{~K}$.

yielded a detection of $\mathrm{CO}$, and hence enabled determination of the $\mathrm{O}$ abundance. Plez et al. (2005) found an unexpectedly high $\mathrm{O}$ enhancement, $[\mathrm{O} / \mathrm{Fe}]$ about +2.2 dex, much higher than the previously assumed value. With the original value for $\epsilon(\mathrm{C})$, this star would not be an extreme $\mathrm{C}$ star, which its spectrum clearly

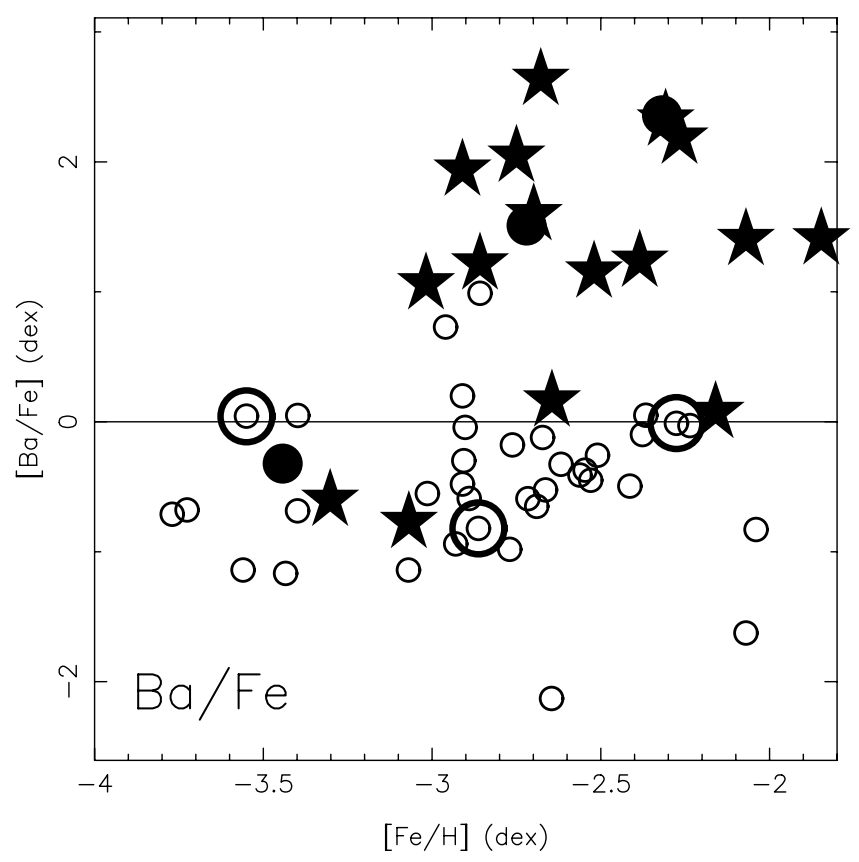

Fig. 9.-Abundance ratio $[\mathrm{Ba} / \mathrm{Fe}]$ as a function of $[\mathrm{Fe} / \mathrm{H}]$ for HES EMP $\mathrm{C}$ stars (large stars) and C-enhanced stars (large filled circles) with detailed abundance analyses. All C-normal stars from the HES analyzed to date by us are shown as small open circles. The additional $\mathrm{C}$ stars from the literature are not shown. Known spectroscopic binaries are circled. 


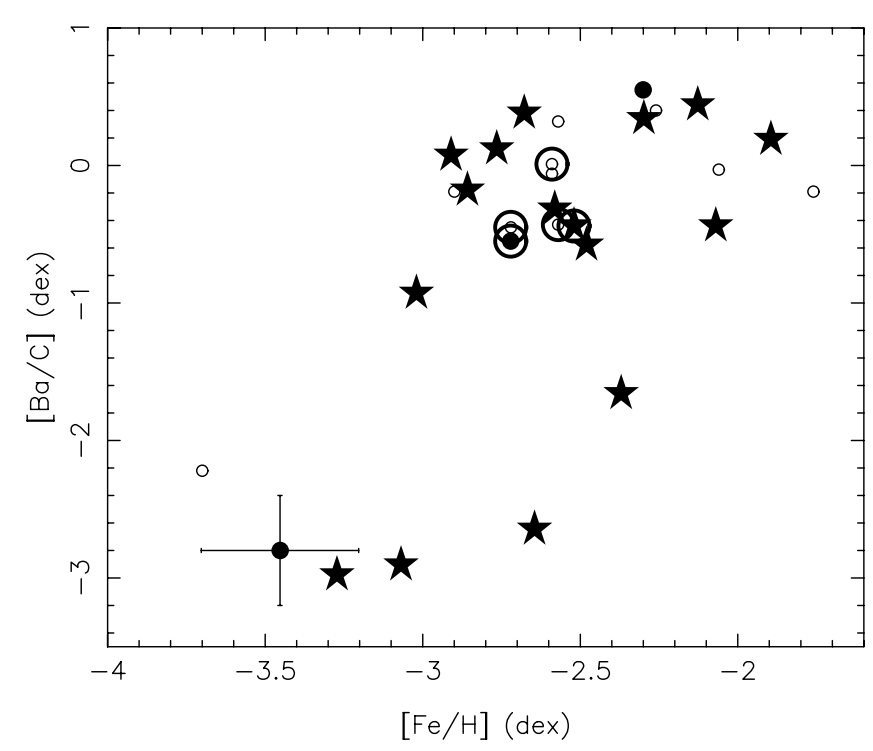

FIG. 10.-Abundance ratio $[\mathrm{Ba} / \mathrm{C}]$ as a function of $[\mathrm{Fe} / \mathrm{H}]$ for HES EMP $\mathrm{C}$ and $\mathrm{C}$-enhanced stars with detailed abundance analyses and for the augmented sample of very metal-poor $\mathrm{C}$ stars from the literature. The symbols are as in Fig. 8; known spectroscopic binaries are circled. Typical error bars are indicated for a single star.

demonstrates that it is. The new higher $\mathrm{O}$ abundance thus in turn led to a revised $[\mathrm{C} / \mathrm{Fe}]$ value of $+3.2 \mathrm{dex}$. The values plotted in the figures for this star (which is included in the additional sample from the literature) are these updated values.

In this context it is important to note that we also in general lack a determination of the $\mathrm{O}$ abundance for the $\mathrm{C}$ stars in our sample (although clearly near-IR spectra of the $\mathrm{CO}$ bands would yield such), and have assumed $[\mathrm{O} / \mathrm{Fe}]$ to be a maximum of $+0.5 \mathrm{dex}$ (or $[\mathrm{C} / \mathrm{Fe}]=-0.8 \mathrm{dex}$ ) in calculating the molecular equilibria for all the $\mathrm{C}$ stars analyzed here. Only one star in our sample has a measured $\mathrm{O}$ abundance; $\mathrm{HE} 1410-0004$ has $[\mathrm{C} / \mathrm{Fe}]=+2.0 \mathrm{dex}$, $[\mathrm{O} / \mathrm{Fe}]=+1.2 \mathrm{dex}$, and $\mathrm{C} / \mathrm{O}=5$. This $\mathrm{O}$ abundance is in accord with the assumption we have chosen to make regarding $[\mathrm{O} / \mathrm{Fe}]$ when no $\mathrm{O}$ abundance is available. If the $\mathrm{O}$ abundance in this star is in fact even lower, which it might be given the marginal detection of the strongest line of the $7770 \AA$ IR triplet, the molecular equilibrium for $\mathrm{CH}$ and for $\mathrm{C}_{2}$ would not change significantly. As the $\mathrm{O}$ abundance is increased from that of $\mathrm{C}$-normal stars, we expect the largest change in the deduced $\mathrm{C}$ abundance (i.e., the larg- est shift in the molecular equilibrium of $\mathrm{CH}$ and $\mathrm{C}_{2}$ ) for $\mathrm{C}$ stars to occur when $\epsilon(\mathrm{O})$ is only slightly less than $\epsilon(\mathrm{C})$. [Recall that $\epsilon(\mathrm{O})$ must be less than $\epsilon(\mathrm{C})$ since these are $\mathrm{C}$ stars.] For changes in $\epsilon(\mathrm{O})$ from the nominal value for HE 1410-0004 given in Table 10 not exceeding a factor of 4 , the change in $\epsilon(\mathrm{C})$ deduced from the $\mathrm{CH}$ band in this star is modest, less than \pm 0.15 dex.

The interpretation of the $\mathrm{CH}$ band strengths as a measure of the $\mathrm{C}$ abundance in the sample $\mathrm{C}$ stars is straightforward, ignoring the issue of the linkage to the assumed $\mathrm{O}$ abundance discussed above. With regard to $\mathrm{C}_{2}$, we look again at Figure 5 (top) (a plot of the absorption at several band heads of $\mathrm{C}_{2}$ vs. $T_{\text {eff }}$ ). Although $\mathrm{C}_{2}$ band strengths were not used to determine the $\mathrm{C}$ abundance, spectral synthesis in the region of the $5160 \AA$ band head with the fixed $\mathrm{CNO}$ abundances $\log \epsilon(\mathrm{C}), \log \epsilon(\mathrm{N})$, and $\log \epsilon(\mathrm{O})=$ $7.56,6.55$, and $7.13 \mathrm{dex}$ (a $\mathrm{C} / \mathrm{O}$ ratio of 2.7; for the value of $f_{00}$, the band oscillator strength adopted by Querci and collaborators) were used to predict the depth of absorption at the $5160 \AA$ band head. The $T_{\text {eff }}, \log g$ pairs were chosen to follow the isochrone for an age of 12 Gyr with $[\mathrm{Fe} / \mathrm{H}]=-2.5$ dex. The result is shown as the solid curve in the top panel of the figure and clearly indicates that increasing absorption at the $\mathrm{C}_{2}$ band head as $T_{\text {eff }}$ decreases is due to the shift in the molecular equilibrium with $T_{\text {eff }}$. Additional curves in this figure are shown for a $\mathrm{C} / \mathrm{O}$ ratio of 1.0 and of $1 / 2.7$, keeping $\epsilon(\mathrm{O})$ fixed, as would occur in a star to which C-rich material is added. The rapid decline in the strength of absorption at the $\mathrm{C}_{2}$ band head is obvious and is due largely to the dependence of $\epsilon\left(\mathrm{C}_{2}\right)$ on $\epsilon\left(\mathrm{C} \mathrm{I}_{\mathrm{I}}\right)^{2}$. Our ability to match the observed strength of the $\mathrm{C}_{2}$ band head in our sample of $\mathrm{C}$ stars shown in Figure 5 by varying only $T_{\text {eff }}$ is consistent with the key result from analysis of the $G$ band of $\mathrm{CH}$ that an approximately constant $\epsilon(\mathrm{C})$ is a satisfactory fit to the existing data on highly $\mathrm{C}$-enhanced stars.

There are no stars in the upper right area of Figure 5. This is, in terms of $\mathrm{C}_{2}$-band detectability, an allowed area. Thus, sufficiently strong bands of $\mathrm{C}_{2}$, equivalent to sufficiently large $\mathrm{C}$ enhancements, do not exist in real stars with $T_{\text {eff }} \sim 6200 \mathrm{~K}$ with their higher continuum flux. The required very large $\mathrm{C}$ enhancements in such hot stars must substantially exceed the constant $\epsilon_{0}(\mathrm{C})$ deduced from the $\mathrm{CH}$ analysis. The maximum $\mathrm{C}_{2}$ band strength, presumably that corresponding to $\epsilon_{0}(\mathrm{C})$, is very weak among the hotter stars in our sample (the main-sequence turnoff region stars), and so stars with lower $\mathrm{C}$ enhancements will simply have no detectable $\mathrm{C}_{2}$. One might wonder why no stars appear in the lower left corner of this plot, where weaker $\mathrm{C}_{2}$ features could easily be detected. This appears to be a consequence of the fact that a $\mathrm{C}$ star must have $\epsilon(\mathrm{C})>\epsilon(\mathrm{O})$; otherwise, oxides will

TABLE 14

Additional C Stars from the Literature

\begin{tabular}{|c|c|c|c|c|c|c|}
\hline ID & References & Binary & $\begin{array}{l}\text { Period } \\
\text { (days) }\end{array}$ & $\begin{array}{c}v_{r} \text { Amplitude } \\
\left(\mathrm{km} \mathrm{s}^{-1}\right)\end{array}$ & $\begin{array}{c}{[\mathrm{Ba} / \mathrm{Fe}]} \\
(\mathrm{dex})\end{array}$ & ${ }^{12} \mathrm{C} /{ }^{13} \mathrm{C}$ \\
\hline G77-61 & 1,2 & Yes & 245 & 20 & $\ldots$ & $\ldots$ \\
\hline CS 22880-074 & 3 & $\ldots$ & $\ldots$ & $\ldots$ & +1.34 & $>40$ \\
\hline CS 22881-036 & 3 & $\ldots$ & $\ldots$ & $\ldots$ & +1.93 & 40: \\
\hline 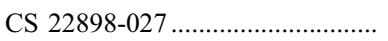 & 3 & $\ldots$ & $\ldots$ & $\ldots$ & +2.27 & $>20$ \\
\hline CS 22942-019 & 3,4 & Yes & 2800 & 5 & +1.92 & 30: \\
\hline 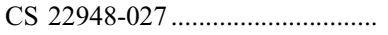 & 3,5 & Yes & 505 & 5 & +1.67 & 14 \\
\hline CS 29497-030 & 6,7 & Yes & 342 & 4 & +2.17 & $>10$ \\
\hline CS 29497-034 & 5 & $\ldots$ & $\ldots$ & $\ldots$ & +2.03 & 12 \\
\hline CS 29498-043 & 8 & $\ldots$ & $\ldots$ & $\ldots$ & -0.45 & 6 \\
\hline CS 30301-015 & 4 & $\ldots$ & $\ldots$ & $\ldots$ & +1.45 & $\ldots$ \\
\hline CS 31062-050 & 4,9 & $\ldots$ & $\ldots$ & $\ldots$ & +2.61 & $\ldots$ \\
\hline
\end{tabular}

References.-(1) Plez \& Cohen 2005; Plez et al. 2005; (2) Dearborn et al. 1986; (3) Preston \& Sneden 2001; (4) Aoki et al. 2002c; (5) Hill et al. 2000; (6) Sivarani et al. 2004; (7) Preston \& Sneden 2000; (8) Aoki et al. 2002b; (9) Johnson \& Bolte 2004. 
TABLE 15

Statistics for Selected Abundance Ratios for the Primary Sample of 16 C Stars from the HeS

\begin{tabular}{|c|c|c|c|c|c|c|}
\hline Species $^{\mathrm{a}}$ & No. Stars ${ }^{\mathrm{b}}$ & $\begin{array}{l}\text { Min. }[X / Y] \\
(\operatorname{dex})\end{array}$ & $\begin{array}{l}\text { Max. }[\mathrm{X} / \mathrm{Y}] \\
(\operatorname{dex})\end{array}$ & $\begin{array}{c}\text { Median }[\mathrm{X} / \mathrm{Y}] \\
(\operatorname{dex})\end{array}$ & $\begin{array}{l}\sigma[\mathrm{X} / \mathrm{Y}] \\
\quad(\mathrm{dex})\end{array}$ & $\begin{array}{c}\text { EMP Dwarfs }{ }^{\mathrm{c}} \\
(\mathrm{dex})\end{array}$ \\
\hline 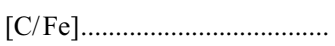 & 16 & 1.22 & 2.52 & 1.93 & 0.31 & $0.2^{\mathrm{d}}$ \\
\hline 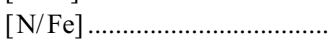 & 14 & 0.47 & 2.52 & 1.75 & 0.59 & $0.0^{\mathrm{d}}$ \\
\hline 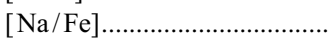 & 3 & 0.03 & 0.48 & 0.37 & 0.23 & $0.41^{\mathrm{e}}$ \\
\hline 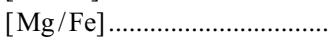 & 12 & 0.04 & 1.04 & 0.55 & 0.27 & 0.56 \\
\hline 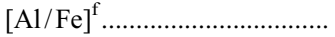 & 10 & -0.55 & 0.88 & 0.27 & 0.39 & -0.09 \\
\hline 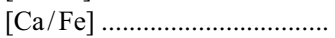 & 14 & 0.11 & 1.12 & 0.42 & 0.34 & 0.31 \\
\hline$\left[\mathrm{Sc}{ }_{\mathrm{II}} / \mathrm{Fe}\right]^{\mathrm{f}} \ldots$. & 5 & 0.05 & 0.67 & 0.48 & 0.26 & 0.24 \\
\hline 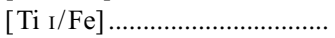 & 14 & 0.03 & 0.57 & 0.36 & 0.15 & 0.36 \\
\hline 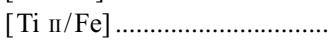 & 15 & 0.11 & 0.96 & 0.36 & 0.26 & 0.36 \\
\hline 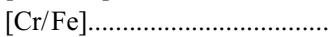 & 14 & -0.70 & -0.06 & -0.32 & 0.21 & -0.23 \\
\hline 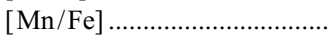 & 12 & -0.72 & 0.03 & -0.31 & 0.23 & -0.59 \\
\hline$[\mathrm{Fe}$ II $/ \mathrm{Fe}$ I $] \ldots \ldots \ldots \ldots \ldots \ldots \ldots \ldots \ldots \ldots \ldots \ldots \ldots$ & 15 & -0.41 & 0.17 & -0.04 & 0.16 & 0.00 \\
\hline 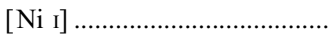 & 4 & -0.65 & 0.02 & -0.32 & 0.27 & -0.02 \\
\hline 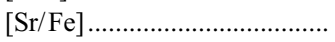 & 12 & -0.98 & 1.68 & 0.32 & 0.69 & -0.19 \\
\hline 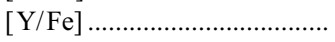 & 11 & -0.01 & 1.40 & 0.55 & 0.39 & $\ldots$ \\
\hline 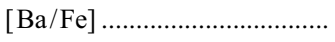 & 16 & -0.78 & 2.63 & 1.30 & 1.01 & -0.20 \\
\hline 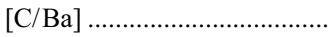 & 16 & -0.44 & 2.98 & 0.43 & 1.15 & $\ldots$ \\
\hline 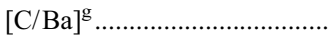 & 12 & -0.44 & 0.93 & 0.15 & 0.49 & $\cdots$ \\
\hline 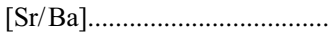 & 12 & -2.23 & 0.21 & -0.94 & 0.69 & $\ldots$ \\
\hline 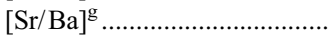 & 8 & -2.23 & -0.85 & -1.11 & 0.51 & $\cdots$ \\
\hline 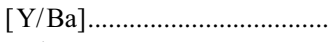 & 9 & -1.73 & -0.72 & -1.21 & 0.35 & $\cdots$ \\
\hline 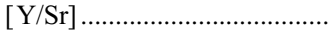 & 8 & -0.28 & 0.60 & -0.03 & 0.32 & $\ldots$ \\
\hline 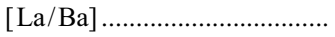 & 8 & -0.71 & 0.10 & -0.32 & 0.26 & $\ldots$ \\
\hline 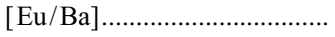 & 4 & -1.47 & -0.34 & -0.82 & 0.46 & $\ldots$ \\
\hline 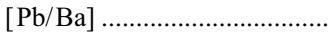 & 7 & 0.14 & 1.21 & 0.79 & 0.34 & $\ldots$ \\
\hline
\end{tabular}

Note.-We include the dwarf C star HE 0007-1832 from Cohen et al. (2004).

a Detection in a minimum of three stars is required.

b Upper limits are ignored.

c Data from Cohen et al. (2004).

d Data for unmixed giants from Spite et al. (2005).

e Data for giants from Cayrel et al. (2004).

${ }^{f}$ Only one line used, with possible blending by molecular features.

g Here we exclude the four $\mathrm{C}$ stars with $[\mathrm{Ba} / \mathrm{Fe}]<0.2$ dex.

dominate the molecular equilibrium. At the solar composition, $\epsilon(\mathrm{C}) / \epsilon(\mathrm{O})$ is about $\frac{1}{2}$. C-normal EMP unevolved and hence unmixed stars (i.e., low-luminosity giants or dwarfs) have $[\mathrm{O} / \mathrm{Fe}]$ about +0.7 dex, while they have $[\mathrm{C} / \mathrm{Fe}]$ about +0.4 dex (for the giants, see, e.g., Spite et al. 2005). A C enhancement of a factor of 4 for a C-normal unmixed EMP star will lead to $\epsilon(\mathrm{C})=\epsilon(\mathrm{O})$, and that required to produce a $\mathrm{C}$ star must be slightly higher. The $\mathrm{C}_{2}$ becomes stronger as the $\mathrm{C}$ enhancement increases above the minimum required to produce a $\mathrm{C}$ star. We suggest that the duration of this phase of $\mathrm{C}$ enhancement is short compared to the age of the EMP C star, and that this phase did not in general occur recently as compared to the timescale for mixing, making this region of Figure 5 unpopulated.

Among more highly evolved EMP and very metal-poor $\mathrm{C}$ stars, we would expect to see some evidence for depletion of $\mathrm{C}$ at the stellar surface as a result of mixing and dredge up, which will depend on the mass included in the mixing region. We use $T_{\text {eff }}$ as a surrogate for the evolutionary stage as the star cools as it moves up the RGB; the M dwarf and EMP star G77-61 is plotted as though its $T_{\text {eff }}$ were $6000 \mathrm{~K}$ to place it at the proper position corresponding to its evolutionary state in this figure. Figure 8 displays $\log \epsilon(\mathrm{C})$ as a function of $T_{\text {eff }}$; the 11 additional Fe-poor $\mathrm{C}$ stars from the literature are included. There is a suggestion in this figure of decreasing $\epsilon(\mathrm{C})$ as $T_{\text {eff }}$ decreases, i.e., as the star moves up the giant branch, reminiscent of mixing and dredge-up phenomena studied among EMP giants by Spite et al. (2005) and among globular cluster giants by Cohen et al. (2005a). The slope of a linear fit to the data in this figure is statistically different from 0.0 at more than the $3 \sigma$ level. The existence of such a correlation, should further work demonstrate conclusively that it is real, would again suggest that the $C$ enhancement could not have occurred recently; sufficient time for $\mathrm{C}$ depletion and mixing in the giant EMP C stars is required.

It is interesting to note that the highest value of ${ }^{12} \mathrm{C} /{ }^{13} \mathrm{C}$ we measured was obtained using the $G$ band of $\mathrm{CH}$ for the hottest and least luminous (and presumably least evolved) of the $\mathrm{C}$ stars with a high-S/N HIRES spectrum. Ryan et al. (2005) compiled ${ }^{12} \mathrm{C} /{ }^{13} \mathrm{C}$ ratios for $\mathrm{Fe}$-poor $\mathrm{C}$-rich stars from the literature. Their compilation also supports the suggestion that there is a general trend of declining ${ }^{12} \mathrm{C} /{ }^{13} \mathrm{C}$ with increasing luminosity. This trend, which needs further confirmation, together with the generally low ${ }^{12} \mathrm{C} /{ }^{13} \mathrm{C}$ ratios, is reproduced by the models of Boothroyd \& Sackmann (1999) as a consequence of deep mixing and "cool bottom processing" after the first and second dredge-up in lowmass red giants. They establish that the latter increases dramatically as $[\mathrm{Fe} / \mathrm{H}]$ decreases. Additional determinations of ${ }^{12} \mathrm{C} /{ }^{13} \mathrm{C}$ for EMP C stars from the $\mathrm{C}_{2}$ band head at $4740 \AA$ will be straightforward and are now underway.

\subsection{Abundance Ratios for Other Elements}

Table 15 gives statistics for selected abundance ratios for the sample of $16 \mathrm{C}$ stars from the HES analyzed here. Upper limits 
are ignored. Only the sample of $16 \mathrm{C}$ stars analyzed here is used to compute the statistical measures given in Table 15. The mean abundance ratios for various elements are compared with those obtained by Cohen et al. (2004) for a large sample of EMP dwarfs, and in some cases to those from the First Stars project at the Very Large Telescope for EMP giants (Cayrel et al. 2004; Spite et al. 2005).

The median $[\mathrm{C} / \mathrm{Fe}]$ is $+1.9 \mathrm{dex}$, with a small dispersion $(0.3 \mathrm{dex})$ about the mean. The lower limit of $\epsilon(\mathrm{C})$ is defined by the requirement that the star be a $\mathrm{C}$ star to be included in the present sample, but the upper bound is not constrained; it is determined by the stellar characteristics themselves. $\mathrm{N}$ is also highly enhanced, with a median $[\mathrm{N} / \mathrm{Fe}]$ of +1.7 dex, only slightly below the median $\mathrm{C}$ enhancement. The scatter is perhaps slightly larger than that seen for $\epsilon(\mathrm{C})$ in Figure 8. Figure 7 (bottom) shows [C/N] as a function of $[\mathrm{Fe} / \mathrm{H}]$. The mean is somewhat higher than the solar value, but there is no obvious trend of $\mathrm{C} / \mathrm{N}$ with $[\mathrm{Fe} / \mathrm{H}]$. Among the giants, there is a suggestion that $\epsilon(\mathrm{N})$ increases and $[\mathrm{C} / \mathrm{N}] \mathrm{de}-$ creases as $T_{\text {eff }}$ decreases and luminosity along the giant branch increases, but the scatter is large, and this may not be statistically significant.

We include in our analysis two of the Mg triplet lines, which lie in a region free of molecular features. Hence, the $\mathrm{Mg}$ abundance should be reliable. ${ }^{13}$ The median abundance ratio $[\mathrm{Mg} / \mathrm{Fe}]$ of our C-star sample agrees well with that of the EMP dwarfs from Cohen et al. (2004), but the range of derived $[\mathrm{Mg} / \mathrm{Fe}]$ is quite large (a factor of 10). The highest value, $[\mathrm{Mg} / \mathrm{Fe}]=+1.04 \mathrm{dex}$ (for HE 0336+0113), is comparable to that of the small number of other extremely Mg-enhanced C-rich stars known, i.e., CS 29498043 discussed by Aoki et al. (2002b) and BS 14934-002 (Aoki et al. 2005). The lowest value of $[\mathrm{Mg} / \mathrm{Fe}]$ among the $\mathrm{C}$ stars in our sample (+0.04 dex, for HE 0212-0557) is comparable to the lowest seen among very metal-poor and EMP stars (see, e.g., the compilation in Fig. 5 of Aoki et al. 2005). [Mg/Fe] almost certainly shows a real range from star to star among EMP stars.

The abundance of Ti should be well determined, as there are many strong Ti II lines in the spectra of these C stars, some of which lie in regions completely free of molecular contamination. $\mathrm{Cr}$ benefits from the strong line at $5206 \AA$, again a region unaffected by molecular features. It is thus gratifying that the $[\mathrm{Ti} / \mathrm{Fe}]$ and $[\mathrm{Cr} / \mathrm{Fe}]$ abundance ratios among the $\mathrm{C}$ stars from the HES show relatively small dispersion, with mean values in good agreement with the results for EMP dwarfs from Cohen et al. (2004). The remaining elements up to the Fe peak suffer from a paucity of unblended lines with strengths sufficiently large for a reliable abundance analysis.

We find that 12 of our $\mathrm{C}$ stars show an enhancement of $\mathrm{Ba}$ (see Fig. 9) and other $s$-process neutron-capture heavy elements approximately equal to that of $\mathrm{C}$. The other four show $[\mathrm{Ba} / \mathrm{C}] \leq$ -1.6 dex, i.e., a strong $\mathrm{C}$ enhancement, with normal heavy elements, as contrasted to enhancement of both $\mathrm{C}$ and the $s$-process elements in the majority of the $\mathrm{C}$ stars. In the full sample of $27 \mathrm{C}$ stars and three $\mathrm{C}$-enhanced dwarfs, six stars do not show a strong Ba enhancement, while $\sim 85 \%$ of the full sample do show a strong $\mathrm{Ba}$ enhancement. Figure 10 shows the $[\mathrm{Ba} / \mathrm{C}]$ ratio for our sample of HES EMP stars. There is a strong suggestion that the stars with low $[\mathrm{Ba} / \mathrm{C}]$ ratios are the most $\mathrm{Fe}$-poor of the sample. The bifurcation into $s$-normal and highly $\mathrm{C}$-enhanced stars is not an artifact of relying on the $\mathrm{Fe}$ abundances, which are decoupled from the $\mathrm{C}$ abundances.

\footnotetext{
13 There is a minor caveat regarding the issue of internal consistency of the $g f$-values between the various $\mathrm{Mg}$ lines discussed in Cohen et al. (2004), but this is a small effect, $\sim 0.2$ dex at most.
}

We can examine whether the process that produces highly enhanced $\mathrm{C}$ in these $\mathrm{C}$ stars also leads to abnormalities in the abundances of other elements beyond those established above, i.e., $\mathrm{CNO}$ and the heavy elements beyond the Fe peak. We define $\Delta(\mathrm{X})$ as the difference between the median $[\mathrm{X} / \mathrm{Fe}]$ in our $\mathrm{C}$-star sample with HIRES abundance analyses and that found for C-normal EMP dwarfs and giants. From the values given in Table 15, for elements from $\mathrm{Na}$ to $\mathrm{Fe}$ we find only two with $|\Delta(\mathrm{X})|>0.25$ dex. These are $\mathrm{Al}[\Delta(\mathrm{Al})=+0.36 \mathrm{dex}]$ and $\mathrm{Mn}$ $[\Delta(\mathrm{Mn})=+0.38 \mathrm{dex}]$. There is only one reliable line for $\mathrm{Al}$ I (at $3961 \AA$ ) and only two for Mn I (two of the three lines of the $4030 \AA$ triplet, ignoring a few very weak lines of Mn that are only rarely detected in the HIRES spectra of these $\mathrm{C}$ stars), and each of these is located in regions of strong $\mathrm{CH}$ absorption. It is likely that there is still some contamination of the atomic features by molecular ones that we were not successful in removing. With this caveat, we thus conclude that the C-star phenomenon in EMP stars is confined to the elements $\mathrm{CNO}$ and to the elements heavier than the Fe peak. The abundance ratios $[\mathrm{X} / \mathrm{Fe}]$ of elements from $\mathrm{Na}$ to $\mathrm{Fe}$ for which we can detect suitable lines are normal.

\subsection{Evidence That s-Process Neutron Capture Dominates among the EMP C Stars}

We discuss here the evidence that enhancement of the neutroncapture elements seen in EMP C stars arises from the $s$-process, with no substantial/detectable contribution from the $r$-process. When we look at the elements beyond the Fe peak, we notice that the median and the mean $[\mathrm{Eu} / \mathrm{Ba}]$ (both about -0.8 dex) closely correspond to that characteristic of the main component of the solar $s$-process given by Arlandini et al. (1999). The detection of large amounts of lead is another clue that the $s$-process is responsible. The median value of $[\mathrm{Pb} / \mathrm{Ba}](+0.79 \mathrm{dex}$, with $\sigma$ about the mean of $0.34 \mathrm{dex}$ ) is close to that of other $s$-process-dominated stars: Sivarani et al. (2004) have compiled all the data for $\mathrm{Pb}$ in such stars available to date (their Table 5 and Fig. 11).

Additional abundance ratios give clues to the detailed behavior of the $s$-process. For example, we find a smaller range in $[\mathrm{Y} / \mathrm{Fe}]$ and in $[\mathrm{Sr} / \mathrm{Fe}]$ than in $[\mathrm{Ba} / \mathrm{Fe}]$, which shows a range of a factor of 1000; this is consistent with metal-poor $s$-processing in asymptotic giant branch (AGB) stars. Busso et al. (1999), for example, predict the $s$-process enhancement will be relatively larger for the second peak elements than for the lighter $s$-process nuclei in stars with lower Fe metallicity. A recent extensive theoretical discussion of the nucleosynthesis of $\mathrm{Sr}, \mathrm{Y}$, and $\mathrm{Zr}$ was given by Travaglio et al. (2004).

Ignoring the upper limits, $\sigma[\mathrm{Y} / \mathrm{Sr}]$ and $\sigma[\mathrm{La} / \mathrm{Ba}]$ are small (0.32 and 0.26 dex, respectively), confirming previous work suggesting that within each of the peaks, the $s$-process element ratios for the Ba-rich EMP C stars are approximately constant for elements within that particular peak, while the variation from star to star of the ratio of the strength of the various peaks is much larger. Aoki et al. (2005) also present relevant data for a sample of 18 very metal-poor stars supporting this.

\subsection{The Ba-poor C Stars}

Figure 10 shows $[\mathrm{Ba} / \mathrm{C}]$ as a function of $\mathrm{Fe}$ metallicity for this sample of $\mathrm{C}$ and $\mathrm{C}$-enhanced stars. Just as was seen in Figure 9, 12 of the $\mathrm{C}$ stars from the HES that we have analyzed show an enhancement of $\mathrm{Ba}$ (and of the other $s$-process neutron-capture heavy elements) approximately equal to that of $\mathrm{C}$. The other four show $[\mathrm{Ba} / \mathrm{C}] \leq-1.2$ dex, i.e., a strong $\mathrm{C}$ enhancement, with more normal heavy elements. Including 10 additional $\mathrm{C}$ stars 
compiled from the literature, 25 of the 30 stars in the full sample of EMP/very metal-poor C-rich stars (83\%) show highly enhanced $\mathrm{Ba}$, while one-sixth have $[\mathrm{Ba} / \mathrm{C}] \leq-1.2 \mathrm{dex}$. It is clear from the evidence described above that the $s$-process is responsible for the enhancement of the heavy neutron-capture elements in these $\mathrm{C}$ stars, when they are highly enhanced. We note that the Ba-poor C stars that are cooler than $T_{\text {eff }}=5700 \mathrm{~K}$ have the same low ${ }^{12} \mathrm{C} /{ }^{13} \mathrm{C}$ ratios as do the Ba-rich $\mathrm{C}$ stars.

We first consider whether the $\mathrm{Ba}$ in the Ba-poor stars is from the $s$ - or the $r$-process. One might argue for the former, claiming that $\mathrm{Ba}$ is in fact enhanced even in the Ba-poor stars. The influence of the very low $\mathrm{Fe}$ metallicity on the heavy neutron-capture rates might give rise to a very low $s$-process production, with the $r$-process making a lower contribution, or even none. However, Figure 9 shows that $[\mathrm{Ba} / \mathrm{Fe}]$ in the Ba-poor stars is consistent with that observed among the C-normal stars from the HES that we have analyzed to date. We know that the Ba in C-normal EMP stars must be largely produced in the $r$-process based on their $[\mathrm{Ba} / \mathrm{Eu}]$ and $[\mathrm{La} / \mathrm{Eu}]$ ratios (e.g., McWilliam et al. 1995a, 1995b; McWilliam 1997, 1998; Simmerer et al. 2004). Thus, we infer that the $\mathrm{Ba}$ in the Ba-poor EMP C stars has its origin in the $r$-process as well.

At first sight, the existence of two more or less distinct classes of EMP C stars suggests that two distinct processes are required to produce the $\mathrm{C}$ stars that are $\mathrm{Ba}$-enhanced and those that are not Ba-rich. Nucleosynthesis within an intermediate-mass AGB star can reproduce the first set of characteristics. If the mass of the EMP C stars is assumed to be the turnoff mass of the halo with an age of $\sim 12 \mathrm{Gyr}$, near $0.8 M_{\odot}$, they are not massive enough to produce $s$-process elements at any time (e.g., see the review by Busso et al. 2004). Also, their $T_{\text {eff }}$ values are too high and the luminosities are too low for our C stars to be AGB stars. Thus, intrinsic nucleosynthesis production and transport to the stellar surface of large amounts of $\mathrm{C}$ is not possible for such unevolved stars.

We suppose instead that the EMP C stars are the former secondaries of binary systems across which mass transfer has occurred. This is the mechanism originally suggested for the $\mathrm{CH}$ stars by McClure (1985), which also have enhanced C and Ba and low Fe metallicities (e.g., Wallerstein \& Greenstein 1964; Vanture 1992), although with $\epsilon(\mathrm{Fe})$ still a factor of 50-100 times higher than the EMP C stars discussed here, the apparent enhancements are not as large in the CH stars. McClure (1984; see also McClure \& Woodsworth 1990) established that essentially all $\mathrm{CH}$ stars are members of binary systems. The higher metallicity Ba stars appear to be another example of the same phenomenon (McClure \& Woodsworth 1990); Bohm-Vitense et al. (2000) have established from UV Hubble Space Telescope spectra the presence of white dwarf companions for several of these stars.

What about the one-sixth of the C-rich stars without heavyelement enhancements? We suggest that there is no need to resort to intrinsic production or any other additional mechanism; in our view, essentially all of these stars could be produced by mass transfer and other phenomena in binary systems. There are several possibilities for explaining these stars within the context of our hypothesis that all EMP C stars are or were binaries. We can ascribe the differing enhancement of the $s$-process elements from $\mathrm{C}$ star to $\mathrm{C}$ star within our sample to some dependence in the nucleosynthetic yields involving, for example, the initial $[\mathrm{Fe} / \mathrm{H}]$ or mass of the original primary star. At the lowest metallicities, Busso et al. (1999; see especially their Fig. 12) predict that when $n$ (Fe seed) becomes very small, there are so many neutrons available for each seed nucleus that the $s$-process runs to completion, with lead the main product and very little Ba enhancement. Lead is the third s-process peak, and $\epsilon(\mathrm{Pb})$ is considerably higher in the Sun than its neighbors in the periodic table. Any heavier elements produced, which are all unstable except for Bi at atomic number 83, decay to lead. Although the prediction of Busso et al. (1999) for the Fe metallicity at which the peak Ba $s$-process production occurs in AGB stars may be slightly too high, their Figure 12 shows a drop of more than a factor of 100 for the predicted $[\mathrm{Ba} / \mathrm{Fe}]$ enhancement as $[\mathrm{Fe} / \mathrm{H}]$ drops 1 dex lower than that at which maximum Ba production occurs.

We attempt to estimate the expected $\mathrm{Pb}$ abundance for an EMP C star assuming the $s$-process runs to completion to see if it is detectable. The highest $[\mathrm{Ba} / \mathrm{Fe}]$ seen among the $\mathrm{C}$ stars in our sample (see Table 15$)$ has $\epsilon(\mathrm{Ba})$ approximately at the solar value for a $\mathrm{C}$ star with $[\mathrm{Fe} / \mathrm{H}]=-2.3 \mathrm{dex}$. We make the reasonable assumption that $s$-process production is proportional to the number of Fe seed nuclei, and assume that all the $s$-process elements, from $\mathrm{Ba}$ to $\mathrm{Pb}$, end up as lead. But all the intervening elements have very low $s$-process abundances; see, e.g., the $s$-process solar abundances for the heavy elements tabulated by Burris et al. (2000). Thus, for an $[\mathrm{Fe} / \mathrm{H}]=-3.5$ dex star, we predict $\epsilon(\mathrm{Pb})$ to be +1.5 dex. This $\mathrm{Pb}$ abundance, which is roughly 2.5 times the solar $\mathrm{Pb}$ abundance, is a very high $\mathrm{Pb}$ abundance for such a low Fe metallicity star. However, it is, as discussed in $\S 4.4$, extremely difficult to detect in a highly $\mathrm{C}$-enhanced [recall that $\epsilon_{0}(\mathrm{C}) \sim \frac{1}{5}$ solar] star with strong molecular bands given that the strongest $\mathrm{Pb}$ I line at optical wavelengths is weak and located in a thicket of $\mathrm{CH}$ features. Thus, verification of this idea through an abundance determination extending to the third $s$-process peak will be very difficult in practice. We do, however, expect in this case that the Ba-poor EMP C stars will be predominantly those of the lowest Fe metallicity, which does appear to be the case in our sample (see Figs. 9 and 10) and in the somewhat smaller sample of Ryan et al. (2005), as well as in that of W. Aoki (2005, private communication).

Another possible explanation for the absence of $s$-process enhancements in some of our EMP C stars is that the neutron flux is strongly reduced in the AGB star, either because of low temperatures in the intershell region or because the ${ }^{13} \mathrm{C}$ pocket fails to be injected into the intershell region of the AGB star, thus restricting the ${ }^{13} \mathrm{C}(\alpha, n){ }^{16} \mathrm{O}$ reaction. This $n$-producing reaction competes with the reaction ${ }^{13} \mathrm{C}(p, \gamma){ }^{14} \mathrm{~N}$. At lower $T$, the latter may dominate, which would reduce the production of neutrons available to create $s$-process elements. The circumstances that might lead to lower $T$ are not clear; perhaps lower Fe metallicity is in some way the dominant factor. In the absence of the neutron flux the $s$-process cannot operate with vigor, thus producing the Ba-poor stars.

We view the trend for the Ba-poor $\mathrm{C}$ stars to be among the most $\mathrm{Fe}$-poor as a fundamental clue to the mechanism(s) involved in producing the Ba-poor $\mathrm{C}$ stars. Any differences in the luminosity distribution of the two groups of $\mathrm{C}$ stars might provide other useful clues for identifying the mechanisms involved. Figure 11 shows a $\left(T_{\text {eff }}, \log g\right)$ diagram for our sample of C-rich stars. Also shown there is the entire sample of EMP candidates from the HES for which we have carried out detailed abundance analyses to date. Our sample is selected from the HES, and stars are chosen for HIRES observations and subsequent abundance analyses solely on the basis of apparent low $[\mathrm{Fe} / \mathrm{H}] .{ }^{14}$ Thus, the distribution of stars, both $\mathrm{C}$-rich and $\mathrm{C}$-normal, along the locus they

\footnotetext{
14 It must be admitted that all the HIRES spectra of C stars in hand as of 2005 August have been analyzed, but not all the spectra of $\mathrm{C}$-normal stars in hand have been analyzed yet. This bias only affects the relative ratio of $\mathrm{C}$-rich to $\mathrm{C}$-normal stars in Fig. 11 but not their distribution along the locus.
} 


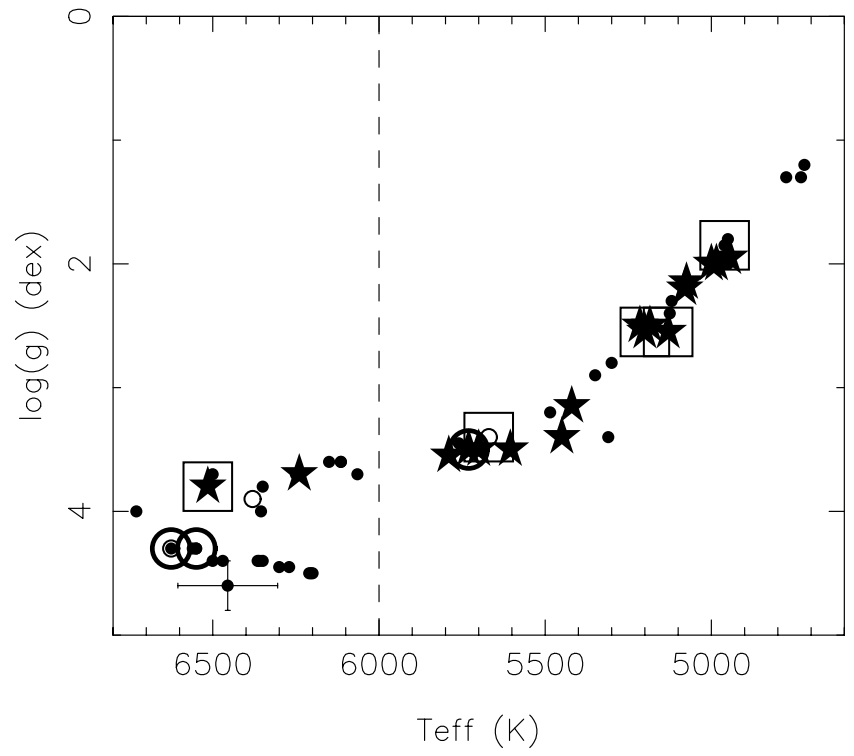

FIG. 11.-H-R diagram ( $T_{\text {eff }}$ Vs. $\log g$ ) for our sample of $16 \mathrm{C}$ stars (stars) and $3 \mathrm{C}$-enhanced stars (open circles). The small filled circles indicate all the other EMP candidates from the HES that we have analyzed to date. The five Ba-poor, $\mathrm{C}$-rich stars from our sample are enclosed in squares. The three known binaries are circled. The additional $\mathrm{C}$ stars from the literature are not shown. Typical error bars are indicated for a single star.

follow in the H-R diagram must represent some folding of the volume surveyed by the HES given the luminosity at each evolutionary stage, the initial mass function for EMP stars, and perhaps selection biases within the HES. The additional $\mathrm{C}$ stars from the literature are not shown in this figure, as they come from various sources, and the selection criteria imposed for highresolution studies are not clear.

Figure 11 suggests that the $\mathrm{C}$ stars of both types are concentrated toward high luminosities and are relatively rare among the turnoff region stars. We ascribe this to a selection effect, as the $G$ band of $\mathrm{CH}$ becomes weaker and more difficult to detect for such hot stars, even if the $\mathrm{C}$ enhancement is very large. The $\mathrm{C}_{2}$ bands become even weaker under such circumstances. Such hot stars can only be picked out as highly $\mathrm{C}$ enhanced from a high-resolution study. Figure 5 demonstrates the weakness of the $\mathrm{C}_{2}$ band in the hot turnoff stars. Low-S/N moderate-resolution spectra are inadequate to securely detect such weak bands. This is the case for the Ba-poor but C-rich star HE 0007-1832 from our sample (this and the other Ba-poor C-rich stars are marked in the figure, as are the known binaries), which is a dwarf $\mathrm{C}$ star whose analysis was published in Cohen et al. (2004). The somewhat hotter main-sequence turnoff at a fixed age for lower metallicity stars $\left(T_{\text {eff }}\right.$ at the turnoff becomes hotter by $150 \mathrm{~K}$ when the Fe metallicity decreases from -2.2 to $-3.2 \mathrm{dex}$ ) makes the $\mathrm{CH}$ and $\mathrm{C}_{2}$ bands in the lowest metallicity stars near the mainsequence turnoff even weaker and more difficult to detect.

Ryan et al. (2005), in a very recent paper discussing the origin of the two classes of $\mathrm{C}$-enhanced metal-poor stars described above, postulate two distinct mechanisms, with mass transfer in an AGB phase of a binary system giving rise to the Ba-rich and $\mathrm{C}$-rich stars, while the Ba-poor, C-rich stars are assigned a completely different origin. However, the discussion given above indicates that there are several plausible scenarios for producing the Ba-poor EMP $\mathrm{C}$ stars within the framework of the binary hypothesis adopted here. We do not find any reason at present to exclude them from also being formed via phenomena involving binary systems.
The path to resolve the origin of the Ba-poor EMP C stars, which is in our view the only remaining area of considerable uncertainty in our scenario, is difficult. It requires assembling a larger sample of such stars, searching within exquisite highresolution spectra for the presence of $\mathrm{Pb}$, and extensive radial velocity monitoring of these stars.

\subsection{Comparison with Disk C Stars}

A comparison of the properties of the EMP C stars with those having Fe metallicity near solar is of interest. Wallerstein \& Knapp (1998) present a review of the luminosities and abundances of the latter. Intrinsic $\mathrm{C}$ stars, stars that produce $\mathrm{C}$ internally and then dredge it up to the stellar surface, are AGB stars with luminosities much higher than those of the EMP C stars in our sample. Lambert et al. (1986) have analyzed such luminous cool disk C stars; their $T_{\text {eff }}$ is considerably lower than the stars studied here. Their sample has $[\mathrm{Fe} / \mathrm{H}] \sim-0.3$ dex and shows only modest $\mathrm{C}$ enhancements (less than a factor of 2 , far smaller than the factor of $\sim 100$ seen in our sample), with no enhancement of $\mathrm{N}$ and with ${ }^{12} \mathrm{C} /{ }^{13} \mathrm{C}$ typically large, $30-70$. The ${ }^{12} \mathrm{C} /{ }^{13} \mathrm{C}$ in these intrinsic $\mathrm{C}$ stars suggests the addition of pure ${ }^{12} \mathrm{C}$ from He burning, with quite different abundance ratios among the $\mathrm{CNO}$ elements and also quite different ${ }^{12} \mathrm{C} /{ }^{13} \mathrm{C}$ ratios from those seen among the much more Fe-poor $\mathrm{C}$ stars studied here. The difference between the $\mathrm{C} / \mathrm{N}$ ratios might arise if the former primary of the binary EMP C stars in our sample had, in the mean, a different stellar mass when it was on the AGB than is typical of disk solar Fe metallicity AGB stars, so as to produce different abundance ratios. Higher mass AGB stars produce higher $\mathrm{C} / \mathrm{N}$ ratios. The predictions from the models of Boothroyd \& Sackmann (1999) are also relevant here, in that a dependence of the nuclear reaction rates and hence the internal production ratios on $[\mathrm{Fe} / \mathrm{H}]$ might also contribute to these differences.

It is now possible to investigate the abundances of $\mathrm{C}$ and $\mathrm{N}$ for luminous AGB C stars in the LMC and the SMC. Preliminary results by Marigo et al. (2003), Matsuura et al. (2005), and Van Loon et al. (2006) suggest that the differences in abundance ratios between these more Fe-poor luminous AGB stars and Galactic disk intrinsic $\mathrm{C}$ stars are small. There is, however, a wellknown decrease in mean luminosity and increase in the $\mathrm{C}$ star to late $\mathrm{M}$ giant ratio as $[\mathrm{Fe} / \mathrm{H}]$ decreases from the Galaxy to the LMC and then to the SMC, first discussed by Blanco et al. (1980). This presumably arises as a smaller amount of $\mathrm{C}$ (of intrinsic origin; these are luminous AGB stars) needs to be added to a very metal-poor star with a fixed $[\mathrm{O} / \mathrm{Fe}]$ ratio to reach $\epsilon(\mathrm{C})=\epsilon(\mathrm{O})$ and so produce a $\mathrm{C}$ star as $[\mathrm{Fe} / \mathrm{H}]$ decreases.

The early $\mathrm{R}$ stars (a type of $\mathrm{C}$ star) are much closer in some of their properties to the Ba-poor EMP C stars found in the HES that are studied here. Dominy $(1984,1985)$ studied their chemical compositions and evolutionary state. (See also the review of Wallerstein \& Knapp [1998].) The R stars are of lower luminosity than the intrinsic AGB C stars, with $M_{\text {bol }} \sim-0.3$ mag and $L \sim 100 L_{\odot}$, and, with $T_{\text {eff }} \sim 4600 \mathrm{~K}$, are warmer than AGB $\mathrm{C}$ stars. Their space density is too high for them to be stars in the He-shell-burning phase of evolution (Scalo \& Miller 1979). They have $[\mathrm{Fe} / \mathrm{H}]$ approximately solar, with moderate $\mathrm{C}$ enhancements $(\sim+0.7 \mathrm{dex})$ and somewhat smaller $\mathrm{N}$ enhancements, but have $\epsilon(\mathrm{O})$ at the solar value. They, like the EMP C stars, have low ${ }^{12} \mathrm{C} /{ }^{13} \mathrm{C}$ ratios. The $\mathrm{R}$ stars do not in general show enhancements of the $s$-process elements. McClure (1997) has demonstrated, via extensive radial velocity monitoring, that they do not appear to be binaries; he suggested that they are coalesced binaries.

Among the various families of high Fe metallicity $\mathrm{C}$ stars, there appears to be a correlation that the stars with highest ${ }^{12} \mathrm{C} /{ }^{13} \mathrm{C}$ are 
those that have strong $s$-process enhancements, while those with the lowest ${ }^{12} \mathrm{C} /{ }^{13} \mathrm{C}$ have little or no enhancement of the elements past the Fe peak. This correlation may be due to the variation with $T$ in the rate of the reaction ${ }^{13} \mathrm{C}(\alpha, n){ }^{16} \mathrm{O}$, which provides the neutrons required for the $s$-process to occur, as compared to that of the reaction ${ }^{13} \mathrm{C}(p, \gamma){ }^{14} \mathrm{~N}$, which suppresses the production of neutrons from ${ }^{13} \mathrm{C}$ burning, or perhaps to some property of the ${ }^{13} \mathrm{C}$ pocket.

\section{IMPLICATIONS OF THE MASS TRANSFER SCENARIO FOR EMP C STARS}

We explore here the consequences of our assumption that mass transfer in binary systems produces all $\mathrm{C}$ stars at all $[\mathrm{Fe} / \mathrm{H}]$ whose luminosities are so low that they cannot be intrinsic $\mathrm{C}$ stars. The stars being discussed here are very metal-poor, so that by adding a small amount of processed material through binary mass transfer, a large change in surface abundances of the secondary star can be produced, which will lead to much more obvious changes in the star's spectral characteristics than would occur at solar metallicity. Furthermore, the efficiency of the complex process of binary mass transfer depends on the mass of the primary star, which affects the mass-loss rate, being higher for higher AGB luminosities, i.e., higher masses of the primary, within certain limits. Also, $d M / d t$ may depend on the metallicity if the mass loss is driven by radiation pressure on dust grains. For a given $d M / d t$ of the AGB star, the accretion rate onto the secondary is a function of the binary separation and other orbital properties. The net result may be a highly variable efficiency for fixed initial $[\mathrm{Fe} / \mathrm{H}]$ and the initial masses of the two components of the binary system.

A key result presented above is the approximately constant $\mathrm{C} / \mathrm{H}$ ratio, $\epsilon(\mathrm{C})=\epsilon_{0}(\mathrm{C})$, in the photospheres of the $\mathrm{C}$ stars in our sample, which we derive from our analysis of their $\mathrm{CH}$ and $\mathrm{C}_{2}$ bands. This is presumably a consequence of the primary nature of $\mathrm{C}$ production in AGB stars. We assume this constant level extends to higher Fe metallicity, although a slight upward trend as $[\mathrm{Fe} / \mathrm{H}]$ increases cannot be ruled out at this point (see Fig. 7). We consider adding this constant $\epsilon_{0}(\mathrm{C})$ to stars of both higher and lower Fe metallicity than those studied here. As $[\mathrm{Fe} / \mathrm{H}]$ rises, the impact of adding additional $\mathrm{C}$ (accompanied by additional $\mathrm{H}$ as well) is diluted. If we assume that $\mathrm{C}$-normal EMP stars have $[\mathrm{C} / \mathrm{Fe}]=+0.3$ dex and $[\mathrm{O} / \mathrm{Fe}]=+0.7 \mathrm{dex}$ and that the stellar photosphere of the star we currently observe consists of equal amounts of its initial material and of material accreted from its AGB companion, then at $[\mathrm{Fe} / \mathrm{H}] \sim-1.4 \mathrm{dex}$, the star, with its additional $\mathrm{C}$, will just achieve $\epsilon(\mathrm{C})=\epsilon(\mathrm{O})$ with the additional $\mathrm{C}$-rich material. This falls to $-2.0 \mathrm{dex}$ if the final photosphere contains 20\% accreted material. More Fe-rich C-normal stars cannot become $\mathrm{C}$ stars through the mass transfer process with our assumptions unless the accreted material comprises more than $50 \%$ of the stellar photosphere.

In this scenario, for higher Fe metallicities we thus expect to see stars that are $\mathrm{C}$-rich but without $\mathrm{C}_{2}$ bands. These presumably correspond to the $\mathrm{CH}$ stars. They occur in the right Fe metallicity range, and essentially all of them were shown by McClure (1984; see also McClure \& Woodsworth 1990) to be binaries. The frequency of $\mathrm{C}$ stars in the HES as a function of $[\mathrm{Fe} / \mathrm{H}]$ to be given in J. G. Cohen et al. (2006b, in preparation) provides further support for this hypothesis. At still higher Fe metallicities, the $\mathrm{C}$ enhancement becomes too small to be noticeable. However, $s$-process production is to first order a secondary process proportional to the number of Fe seed nuclei (i.e., to [Fe/H]). Thus, $s$-enhancement (i.e., the high levels of $[s / \mathrm{Fe}]$ ) will still be present at high $\mathrm{Fe}$ metallicity, although the details of the nucleosynthesis may shift the relative production of the $s$-process nuclei toward the first peak at $\mathrm{Sr}$ (see, e.g., Busso et al. 1999). Such stars presumably correspond to the Ba stars, which are of higher Fe metallicity than the CH stars. According to McClure \& Woodsworth (1990; see also Luck \& Bond 1991), the Ba stars are another example of the same phenomenon of mass transfer in binary systems.

The situation at lower Fe metallicities was explored in $\S$ 5.4. We expect, as described earlier, the $s$-process to run through to lead, which will be extremely difficult to detect, with very low production of the more easily detected $s$-process elements such as $\mathrm{Sr}, \mathrm{Ba}$, and $\mathrm{La}$.

The low ${ }^{12} \mathrm{C} /{ }^{13} \mathrm{C}$ ratios seen in these EMP $\mathrm{C}$ stars, both $\mathrm{Ba}-$ enhanced and Ba-poor, provide another important clue. They, combined with the high $\mathrm{C} / \mathrm{N}$ ratios, suggest that a two-phase process is required. First, mass transfer across the binary system from a low Fe metallicity AGB star with intrinsic production of $\mathrm{C}$ (and hence a high ${ }^{12} \mathrm{C} /{ }^{13} \mathrm{C}$ ratio) occurs. This is then followed by a phase of mixing combined with "cold bottom burning" as described by Boothroyd \& Sackmann (1999) to produce the observed $\mathrm{C} / \mathrm{N}$ and ${ }^{12} \mathrm{C} /{ }^{13} \mathrm{C}$ ratios. (See Gratton et al. [2000] for a description of the consequences of this mixing process in more metal-rich C-normal field stars.) Since the degree of C depletion appears to depend on the luminosity of the $\mathrm{C}$ star we observe today, that part of the processing cannot have occurred in the donor star of the binary.

\subsection{Binarity}

We have suggested that all EMP C stars (i.e., those with $-4 \mathrm{dex} \lesssim[\mathrm{Fe} / \mathrm{H}] \lesssim-2 \mathrm{dex})$ are the original secondary stars of binary systems in which mass transfer occurred. We have further suggested that this mass transfer from an AGB primary can produce the abundance anomalies we see among the EMP C stars, specifically the high enhancement of $s$-process elements among $\sim 85 \%$ of these $\mathrm{C}$ stars. Those very metal-poor/EMP C stars with low or no $s$-process enhancement are cases in which some factor, most likely the low Fe metallicity of the primary, while still producing, mixing to its surface, and transferring to the secondary star ample amounts of carbon, did not achieve such for the easily detectable heavy neutron-capture element $\mathrm{Ba}$.

We consider here whether the statistics of binary detection among very metal-poor $\mathrm{C}$ stars can support our hypothesis that all of these $\mathrm{C}$ stars were once binaries. We expect most/all of them to still be binaries with (invisible) white dwarf companions. The HES C stars of our sample are themselves not suitable for this purpose. They were only recently discovered to be interesting stars, and most have only been observed for a single epoch. They are in general faint for high-dispersion spectroscopic analysis. There were no radial velocity monitoring programs for such stars until very recently. Even so, we have already found three confirmed binaries in our samples of candidate EMP stars from the HES.

So we look instead at the sample of additional $\mathrm{C}$ stars from the literature. These stars are in general brighter than the HES C stars in our sample, and they have been known as interesting objects for timescales of several years to a decade, giving more opportunity for radial velocity monitoring. Table 14 indicates which of these are known binaries and gives their periods and $v_{r}$ amplitudes. Four of these $11 \mathrm{C}$ stars are confirmed binaries, consistent with the very preliminary results of the $v_{r}$ monitoring program of Tsangarides et al. (2004) for $s$-process-enhanced C stars.

Although the sample is small, considering the lack of suitable long-term radial velocity monitoring programs, the length of the typical period, the small velocity amplitudes, the faintness of the stars, and the relatively short time they have been known to be 
interesting, we find our detection rate for binaries among very metal-poor and EMP C stars to be consistent with all such stars being binaries; Monte Carlo simulations by Lucatello et al. (2005) support this. There is as yet insufficient $v_{r}$ monitoring data for the small fraction of C-enhanced stars without $s$-process enhancement to assess their binarity.

\section{SUMMARY}

We have studied a sample of $16 \mathrm{C}$ stars from the EMP candidate lists of the HES using high-dispersion spectra from HIRES at Keck and new optical photometry. We have carried out a detailed abundance analysis using a $T_{\text {eff }}$ scale based on $V-I, V-J$, and $V-K$ colors, while avoiding the effects of the molecular bands as much as possible. Earlier $T_{\text {eff }}$ scale problems affecting the Fe metallicity deduced for EMP stars as hot as $6000 \mathrm{~K}$ by the HES (and, until recently, the HK survey) were solved by changing from $B-V$ to $\mathrm{H} \delta$ as a $T_{\text {eff }}$ indicator. Our results provide a broad database to establish the Fe metallicity for EMP C stars. We find that the Fe metallicities for the cooler $\mathrm{C}$ stars $\left(T_{\text {eff }} \sim 5100 \mathrm{~K}\right)$ are still being underestimated by a factor of $\sim 10$ by the current standard HES (and until very recently HK survey) tools. This is due to strong molecular absorption primarily in the red continuum bandpass of the HP2 index, which measures the strength of $\mathrm{H} \delta$ and acts as an indicator of $T_{\text {eff }}$. The results presented here provided crucial supporting data used by Cohen et al. (2005b) to derive the frequency of $\mathrm{C}$ stars among EMP stars.

Carbon abundances in these very metal-poor stars appear to be constant, independent of Fe metallicity, at about one-fifth the solar value. The $\mathrm{C}$ abundances show marginal evidence of decreasing with decreasing $T_{\text {eff }}$ or increasing luminosity, presumably due to mixing and dredge-up of C-depleted material. Such $\mathrm{C}$ depletion is seen among "normal" halo field giants over a wide range of metallicity for sufficiently evolved stars with luminosities brighter than that of the RGB bump, which is high on the RGB. Nitrogen is also highly enhanced in the EMP C stars. Among the elements studied here, abundance anomalies in these stars appear to be confined to $\mathrm{CNO}$ and to those heavier than the Fe peak.

$\mathrm{C}$ enhancement in this sample is associated with strong enhancement of $s$-process heavy nuclei for 12 of the 16 stars, with $[\mathrm{C} / \mathrm{Ba}]$ about -0.1 dex with small scatter. The remaining four $\mathrm{C}$ stars from the HES show no evidence for enhancement of the heavy elements, with Ba providing the strongest constraint, $[\mathrm{Ba} / \mathrm{Fe}] \leq+0.20 \mathrm{dex}$, for each of the four stars. When 11 additional C stars, mostly from the HK survey, with recently published detailed abundance analyses are added, the same separation is seen, with $\sim 85 \%$ of the stars having $[\mathrm{C} / \mathrm{Ba}]$ almost solar.

Very high enhancements of lead are detected in some of the $\mathrm{C}$ stars with highly enhanced $\mathrm{Ba}$. The ratio $\mathrm{Ba} / \mathrm{Eu}$, the high $\mathrm{Pb}$ abundances, and the high ratios of diagnostic elements in the second to the first $s$-process peak for C stars in our sample demonstrate that the $s$-process is responsible for the enhancement of the heavy elements for most of the $\mathrm{C}$ stars in our sample. The mostly low ${ }^{12} \mathrm{C} /{ }^{13} \mathrm{C}$ ratios inferred from both the $G$ band of $\mathrm{CH}$ and the $4740 \AA$ band of $C_{2}$, in which the isotope ratio is particularly easy to measure, as well as the high $\mathrm{N}$ enhancements, suggest that the bulk of the stellar envelope of these stars has been processed through the $\mathrm{CN}$ cycle of proton burning. Our data for the Ba-rich $\mathrm{C}$ stars supports the suggestion that the abundance ratios for elements within a given $s$-process peak are to first order constant, while the ratio of the strength of the various peaks shows larger star-to-star variations.

The similarities and differences of the properties of the EMP $\mathrm{C}$ stars to those of various types of near-solar $[\mathrm{Fe} / \mathrm{H}]$ disk $\mathrm{C}$ stars are discussed. In particular, the early $\mathrm{R}$ stars show low ${ }^{12} \mathrm{C} /{ }^{13} \mathrm{C}$ ratios and no excess of the heavy elements, reminiscent of the Ba-poor EMP C stars found (at a low rate) in our sample.

The abundance ratios we derive are used to discuss the origin of the C-rich stars among EMP stars. We suggest that both the $s$-process-enhanced and $\mathrm{Ba}$-normal $\mathrm{C}$ stars result from phenomena associated with binary stars. The Ba-rich EMP C stars presumably formed as secondaries in a mass transfer binary system with an AGB primary. This was followed by proton burning at moderate $T$ to reduce ${ }^{12} \mathrm{C} /{ }^{13} \mathrm{C}$ and increase the $\mathrm{C} / \mathrm{N}$ ratio. The implications of this hypothesis for stars of both higher and lower Fe metallicity than those in the present sample are discussed. Several possible origins for the small minority of Ba-poor EMP $\mathrm{C}$ stars are suggested. In the most metal-poor stars, Busso et al. (1999) predict that the $s$-process runs to completion through the Ba peak to the heaviest stable element, lead, leaving little or no apparent Ba excess. Heavier elements (all unstable except Bi) mostly decay to lead as well. The predicted $\epsilon(\mathrm{Pb})$ in a $[\mathrm{Fe} / \mathrm{H}]=$ -3.5 dex star, while very high for a star with such a low Fe metallicity, will be very difficult to detect. Another possibility for explaining the Ba-poor EMP $\mathrm{C}$ stars is a possible lack of neutrons due to ${ }^{13} \mathrm{C}$ burning via ${ }^{13} \mathrm{C}(p, \gamma){ }^{14} \mathrm{~N}$ instead of via ${ }^{13} \mathrm{C}(\alpha, n){ }^{16} \mathrm{O}$. The former dominates at lower $T$, while the latter provides the neutrons required for the $s$-process to occur. If either of these suggestions is correct, the Ba-poor $\mathrm{C}$ stars should have lower Fe metallicities in the mean than the Ba-rich $\mathrm{C}$ stars, which does appear to be the case in our sample. The frequency of known binaries among the samples appears consistent with our hypothesis for the origin of EMP C stars given the lack of long-term radial velocity monitoring programs, the long periods, the low velocity amplitudes, and other characteristics of the stars.

We thus see no reason at present to exclude the scenario adopted here, that all the EMP C stars are formed via phenomena involving binary systems. For old stars of low Fe metallicity, several mechanisms described above may lead to $\mathrm{C}$ stars with little or no $s$-process enhancement, such as is occasionally seen in our sample. For old stars in binary mass transfer systems of higher $[\mathrm{Fe} / \mathrm{H}]$ than those considered here, a progression with increasing $[\mathrm{Fe} / \mathrm{H}]$ from $\mathrm{C}$ stars to $\mathrm{CH}$ stars and finally to Ba stars is predicted for a constant donor $\epsilon_{0}(\mathrm{C})$, which successfully reproduces several key observed characteristics of the behavior of C-rich stars in the Galaxy.

The entire Keck HIRES user community owes a huge debt to Jerry Nelson, Gerry Smith, Steve Vogt, and many other people who have worked to make the Keck telescope and HIRES a reality and to operate and maintain the Keck Observatory. We are grateful to the W. M. Keck Foundation for the vision to fund the construction of the W. M. Keck Observatory. We are grateful to W. Aoki for providing his Subaru HDS spectra of selected $\mathrm{C}$ stars to verify our ${ }^{13} \mathrm{CH}$ line list. We thank G. Wasserburg for helpful discussions and moral support. This publication makes use of data products from the Two Micron All Sky Survey, which is a joint project of the University of Massachusetts and the Infrared Processing and Analysis Center/California Institute of Technology, funded by the National Aeronautics and Space Administration and the National Science Foundation. J. G. C. is grateful for partial support from NSF grants AST 02-05951 and AST 05-07219. She is grateful for funds from the Ernest Fullam Award of the Dudley Observatory for help in initiating this work. N. C. and F. J. Z. acknowledge support from Deutsche Forschungsgemeinschaft through grant Re 353/44. N. C. is also supported by a Henri Chretien International Research Grant administered by the American Astronomical Society. 
Alonso, A., Arribas, S., \& Martínez-Roger, C. 1996, A\&A, 313, 873 1999, A\&AS, 140, 261

Amiot, C. 1983, ApJS, 52, 329

Anders, E., \& Grevesse, N. 1989, Geochim. Cosmochim. Acta, 53, 197

Aoki, W., Norris, J. E., Ryan, S. G., Beers, T. C., \& Ando, H. 2002a, ApJ, 567, 1166

2002b, ApJ, 576, L141

Aoki, W., Ryan, S. G., Norris, J. E., Beers, T. C., Ando, H., \& Tsangarides, S. 2002c, ApJ, 580, 1149

Aoki, W., et al. 2001, ApJ, 561, 346

2005, ApJ, 632, 611

Arlandini, C., Käppeler, F., Wisshak, K., Gallino, R., Lugaro, M., Busso, M., \& Straniero, O. 1999, ApJ, 525, 886

Asplund, M., Grevesse, N., Sauval, A. J., Allende Prieto, C., \& Bloome, R. 2005, A\&A, 431, 693

Asplund, M., Grevesse, N., Sauval, A. J., Allende Prieto, C., \& Kisselman, D. 2004, A\&A, 417, 751

Asplund, M., Nordlund, Å., Trampedach, R., Prieto, C. A., \& Stein, R. F. 2000, A\&A, 359, 729

Barbuy, B., et al. 1997, A\&A, 317, L63

Baumüller, D. G., Butler, K., \& Gehren, T. 1998, A\&A, 338, 637

Baumüller, D. G., \& Gehren, T. 1997, A\&A, 325, 1088

Beers, T. C., \& Christlieb, N. 2005, ARA\&A, 43, 531

Beers, T. C., Preston, G. W., \& Shectman, S. 1985, AJ, 90, 2089 1992, AJ, 103, 1987

Beers, T. C., Rossi, S., Norris, J. E., Ryan, S., \& Shefler, T. 1999, AJ, 117, 981

Blanco, V. M., McCarthy, M. F., \& Blanco, B. M. 1980, ApJ, 242, 938

Bohm-Vitense, E., Carpenter, K., Robinson, R., Ake, T., \& Brown, J. 2000, ApJ, 533, 969

Bonifacio, P., Molaro, P., Beers, T. C., \& Vladilo, G. 1998, A\&A, 332, 672

Boothroyd, A. I., \& Sackmann, I. J. 1999, ApJ, 510, 232

Burris, D. L., Pilachowski, C. A., Armandroff, T. E., Sneden, C., Cowan, J. J., \& Roe, H. 2000, ApJ, 544, 302

Busso, M., Gallino, R., \& Wasserburg, G. J. 1999, ARA\&A, 37, 239

Busso, M., Straniero, O., Gallino, R., \& Abis, C. 2004, in Origin and Evolution of the Elements, ed. A. McWilliam \& M. Rauch (New York: Cambridge Univ. Press), 67

Carretta, E., Gratton, R. G., Cohen, J. G., Beers, T. C., \& Christlieb, N. 2002, AJ, 124, 481

Cayrel, R., et al. 2004, A\&A, 416, 1117

Christlieb, N. 2003, Rev. Mod. Astron., 16, 191

Christlieb, N., Gustafsson, B., Korn, A. J., Barklem, P. S., Beers, T. C., Bessell, M. S., Karlsson, T., \& Mizuno-Wiedner, M. 2004, ApJ, 603, 708

Cohen, J. G., Briley, M. M., \& Stetson, P. B. 2005a, AJ, 130, 1177

Cohen, J. G., Christlieb, N., Beers, T. C., Gratton, R. G., \& Carretta, E. 2002, AJ, 124, 470

Cohen, J. G., Christlieb, N., Qian, Y. Z., \& Wasserburg, J. G. 2003, ApJ, 588, 1082

Cohen, J. G., et al. 2004, ApJ, 612, 1107 2005b, ApJ, 633, L109

Cutri, R. M., et al. 2003, Explanatory Supplement to the 2MASS All-Sky Data Release (Pasadena: Caltech), http://www.ipac.caltech.edu/2mass/releases/ allsky/doc/explsup.html

Dearborn, D. S. P., Liebert, J., Aaronson, M., Dahn, C. C., Harrington, R., Mould, J., \& Greenstein, J. L. 1986, ApJ, 300, 314

Dominy, J. F. 1984, ApJS, 55, 27 1985, PASP, 97, 1104

Frebel, A., et al. 2005, Nature, 434, 871

Gratton, R. G., Sneden, C., Carretta, E., \& Bragaglia, A. 2000, A\&A, 354, 169

Grevesse, N., \& Sauval, A. J. 1998, Space Sci. Rev., 85, 161

Hill, V., et al. 2000, A\&A, 353, 557

Hobbs, L. M., Thorburn, J. A., \& Rebull, L. M. 1999, ApJ, 523, 797

Holweger, H. 2001, in AIP Conf. Proc. 598, Solar and Galactic Composition, ed. R. F. Wimmer-Schweingruber (New York: AIP), 23

Houdashelt, M. L., Bell, R. A., \& Sweigart, A. V. 2000, AJ, 119, 1448

Johnson, J. J., \& Bolte, M. 2002, ApJ, 579, L87 2004, ApJ, 605, 462

Keenan, P. C., \& McNeil, R. C. 1976, An Atlas of Spectra of the Cooler Stars: Types G, K, M, S, and C (Columbus: Ohio State Univ. Press)

\section{EFERENCES}

Kisselman, D. 2001, NewA Rev., 45, 559

Kurucz, R. L. 1993, Kurucz CD-ROM 13, ATLAS9 Stellar Atmosphere Programs and $2 \mathrm{~km} / \mathrm{s}$ Grid (Cambridge: $\mathrm{SAO}$ )

Lambert, D. L., Gustaffson, B., Eriksson, H., \& Hinkle, K. H. 1986, ApJS, 62, 373

Lawler, J. E., Bonvallet, G., \& Sneden, C. 2001a, ApJ, 556, 452

Lawler, J. E., Wickliffe, M. E., Den Hartog, E. A., \& Sneden, C. 2001b, ApJ, 563,1075

Lucatello, S., Gratton, R., Cohen, J. G., Beers, T. C., Christlieb, N., Carretta, E., \& Ramírez, S. 2003, AJ, 125, 875

Lucatello, S., Tsangarides, S., Beers, T. C., Carretta, E., Gratton, R. G., \& Ryan, S. G. 2005, ApJ, 625, 825

Luck, R. E., \& Bond, H. E. 1991, ApJS, 77, 515

Marigo, P., Bernard-Salas, J., Pottasch, S. R., Tielens, A. G. G. M., \& Wesselius, P. R. 2003, A\&A, 409, 619

Marsteller, B., Beers, T. C., Rossi, S., Christlieb, N., Bessell, M., \& Rhee, J. 2005, Nucl. Phys. A, 758, 312

Matsuura, M., et al. 2005, A\&A, 434, 691

McClure, R. D. 1984, ApJ, 280, L31

1985, J. R. Astron. Soc. Canada, 79, 277 1997, PASP, 109, 256

McClure, R. D., \& Woodsworth, A. W. 1990, ApJ, 352, 709

McWilliam, A. 1997, ARA\&A, 35, 503 1998, AJ, 115, 1640

McWilliam, A., Preston, G. W., Sneden, C., \& Searle, L. 1995a, AJ, 109, 2757

McWilliam, A., Preston, G. W., Sneden, C., \& Shectman, S. 1995b, AJ, 109, 2736

Norris, J. E., Ryan, S., \& Beers, T. C. 1997, ApJ, 489, L169

Oke, J. B., \& Gunn, J. E. 1982, PASP, 94, 586

Plez, B., \& Cohen, J. G. 2005, A\&A, 434, 1117

Plez, B., Cohen, J. G., \& Melendez, J. 2005, in IAU Symp. 228, From Lithium to Uranium: Elemental Tracers of Early Cosmic Evolution, ed. V. Hill, P. Francois, \& F. Primas (New York: Cambridge Univ. Press), 267

Preston, G. W., \& Sneden, C. 2000, AJ, 120, 1014

2001, AJ, 122, 1545

Prochaska, J. X., Naumov, S. O., Carney, B. W., McWilliam, A., \& Wolfe, A. M. 2000, AJ, 120, 2513

Querci, F., Querci, M., \& Kunde, V. G. 1971, A\&A, 15, 256

Querci, F., Querci, M., \& Tsuji, T. 1974, A\&A, 31, 265

Ramírez, S. V., Cohen, J. G., Buss, J., \& Briley, M. M. 2001, AJ, 122, 1429

Rossi, S., Beers, T. C., Sneden, C., Sevastyanenko, T., Rhee, J., \& Marsteller, B. 2005, AJ, 130, 2804

Ryan, S. G., Aoki, W., Norris, J. E., \& Beers, T. C. 2005, ApJ, 635, 349

Scalo, J. M., \& Miller, G. E. 1979, ApJ, 233, 596

Simmerer, J., Sneden, C., Cowan, J. J., Collier, J., Woolf, V. M., \& Lawler, J. E. 2004, ApJ, 617, 1091

Sivarani, T., et al. 2004, A\&A, 413, 1073

Skrutskie, M. F., et al. 2006, AJ, 131, 1163

Sneden, C. 1973, Ph.D. thesis, Univ. Texas

Spite, M., et al. 2005, A\&A, 430, 655

Takeda, Y. 2003, A\&A, 402, 343

Takeda, Y., Zhao, G., Takad-Hidai, M., Chen, Y. Q., Saito, Y., \& Zhang, H. W. 2003, Chinese J. Astron. Astrophys., 3, 316

Travaglio, C., Gallino, R., Arnone, E., Cowan, J., Jordan, F., \& Sneden, C. 2004, ApJ, 601, 864

Tsangarides, S., Ryan, S. G., \& Beers, T. C. 2004, Mem. Soc. Astron. Italiana, 75,772

Urdahl, R. S., Bao, Y., \& Jackson, W. M. 1991, Chem. Phys. Lett., 178, 425

Van Eck, S., Goriely, S., Jorissen, A., \& Plez, B. 2003, A\&A, 404, 291

Van Loon, J. T., Stanimirovic, S., Evans, A., \& Muller, E. 2006, MNRAS, 365 , 1277

Vanture, A. D. 1992, AJ, 104, 1997

Vogt, S. E., et al. 1994, Proc. SPIE, 2198, 362

Wallace, L., Hinkle, K., \& Livingston, W. 1998, NSO Tech. Rep. 98-001 (Kitt Peak: NSO), http://ftp.noao.edu.fts/visatl/README

Wallerstein, G., \& Greenstein, J. L. 1964, ApJ, 139, 1163

Wallerstein, G., \& Knapp, G. R. 1998, ARA\&A, 36, 369

Yi, S., Demarque, P., Kim, Y.-C., Lee, Y.-W., Ree, C., Lejeune, Th., \& Barnes, S. 2001, ApJS, 136, 417 